\title{
A theory of induction and classification of tensor $\mathrm{C}^{*}$-categories
}

\author{
Claudia Pinzari and John E. Roberts
}

\begin{abstract}
This paper addresses the problem of describing the structure of tensor $\mathrm{C}^{*}$-categories $\mathcal{M}$ with conjugates and irreducible tensor unit. No assumption on the existence of a braided symmetry or on amenability is made. Our assumptions are motivated by the remark that these categories often contain non-full tensor $\mathrm{C}^{*}$-subcategories with conjugates and the same objects admitting an embedding into the Hilbert spaces. Such an embedding defines a compact quantum group by Woronowicz duality. An important example is the Temperley-Lieb category canonically contained in a tensor $\mathrm{C}^{*}$-category generated by a single real or pseudoreal object of dimension $\geq 2$. The associated quantum groups are the universal orthogonal quantum groups of Wang and Van Daele.

Our main result asserts that there is a full and faithful tensor functor from $\mathcal{M}$ to a category of Hilbert bimodule representations of the compact quantum group. In the classical case, these bimodule representations reduce to the $G$-equivariant Hermitian bundles over compact homogeneous $G$-spaces, with $G$ a compact group. Our structural results shed light on the problem of whether there is an embedding functor of $\mathcal{M}$ into the Hilbert spaces. We show that this is related to the problem of whether a classical compact Lie group can act ergodically on a non-type I von Neumann algebra. In particular, combining this with a result of Wassermann shows that an embedding exists if $\mathcal{M}$ is generated by a pseudoreal object of dimension 2 .
\end{abstract}

Mathematics Subject Classification (2010). 46L55, 81R15, 58B32, 46L65, 46M15.

Keywords. Tensor $\mathrm{C}^{*}$-categories, quasi-tensor functors, compact quantum groups, ergodic $\mathrm{C}^{*}$-actions, Hilbert bimodule representations, induced $\mathrm{C}^{*}$-bimodules.

\section{Contents}

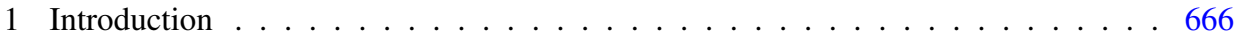

2 Notation and preliminaries . . . . . . . . . . . . . . . . . 669

3 Bimodule representations of compact quantum groups . . . . . . . . . . . . . . 675

4 The induced C*-bimodules for compact groups . . . . . . . . . . . . . . 677

5 Full bimodule representations . . . . . . . . . . . . . . . . . . . . . . . . 679

6 Main results . . . . . . . . . . . . . . . . . . . . . . 6 682

7 Algebraic bimodules from pairs of functors . . . . . . . . . . . . . . . 687

8 The induced Hilbert bimodule representations ．. . . . . . . . . . . . . . . . . 691

9 Extending Ind to a full tensor functor . . . . . . . . . . . . . . . . . . . . . 697

10 An adjoint pair of functors . . . . . . . . . . . . . . . . . 702

11 Full bimodule representations from group actions . . . . . . . . . . 703 
12 Tensorial properties of the evaluation functor . . . . . . . . . . . . 711

13 Appendix . . . . . . . . . . . . . . . . . . 714

References . . . . . . . . . . . . . . . . . 716

\section{Introduction}

By Tannaka-Krein duality theory, a semisimple rigid tensor category admits an embedding functor into the category of finite-dimensional vector spaces if and only if it is the representation category of a quantum group. There are variants of this result depending on the framework under consideration. This characterization does however not allow one to tell whether a given tensor category admits such an embedding.

A positive result, motivated by algebraic QFT, asserts that a symmetric tensor $\mathrm{C}^{*}$ category with conjugates $\mathcal{M}$, is equivalent, after completion under direct sums and subobjects, to the symmetric tensor $\mathrm{C}^{*}$-category of finite-dimensional representations of a unique compact group [11]. There is a similar well-known result in the context of algebraic geometry [8].

However, different finite groups may have equivalent representation categories [12], [17], so the symmetry is crucial for uniqueness.

Another positive result is a theorem of [14], asserting that a semisimple rigid tensor category with finitely many irreducibles is equivalent to a representation category of a finite-dimensional, but not unique, weak Hopf algebra in the sense of [5], see also [30]. However, this theorem does not say anything about whether the weak Hopf algebras can be chosen to be a quantum group or even a group. Moreover, this approach does not generalize easily to categories with infinitely many irreducibles, as difficulties of an analytic nature arise.

In this paper we consider the analytic framework of tensor $\mathrm{C}^{*}$-categories with conjugates. Now, an embedding functor is naturally required to take values in the category Hilb of Hilbert spaces. Here, the quantum groups of the Tannaka-Krein duality theory are compact quantum groups [50]. However, even in this case, there are situations where there can be no such embedding.

For example, if a tensor $\mathrm{C}^{*}$-category with conjugates $\mathcal{M}$ has an object with an intrinsic dimension strictly between 1 and 2, it obviously cannot be embedded into Hilb. These cases are often related to quantum groups at roots of unity by Jones fundamental result on the restriction of the index values [18], [48]. Ocneanu [29] indicated that they should be understood as 'quantum subgroups' of the deformed $\mathrm{SU}(2)$, a program developed in [19].

But other classes are known even when there are objects with intrinsic dimension $>2$. For example, consider an irreducible inclusion of $\mathrm{II}_{1}$ factors $N \subset M$ with finite Jones index $[M: N]$ and consider the tensor $\mathrm{C}^{*}$-category of $N$-bimodules generated by ${ }_{N} M_{N}$. This category has conjugates and the intrinsic dimension of the generator ${ }_{N} M_{N}$ is $[M: N]$, hence $\geq 2$ if the inclusion is proper, again by the restriction of the index values. By an easy consequence of Popa's work [38], this category cannot be 
embedded in Hilb whenever the index is not an integer and the inclusion is amenable in the sense of Popa, see [36]. There is a similar result for an amenable object in a tensor $\mathrm{C}^{*}$-category with non-integral dimension [22]. (The precise relation between the two notions of amenability has not been clarified.)

On the other hand, compact quantum groups provide examples of tensor $\mathrm{C}^{*}$ categories with conjugates that are embedded but not amenable, as the intrinsic dimensions of unitary representations are often not integral.

This paper addresses the problem of describing the structure of tensor $\mathrm{C}^{*}$-categories with conjugates and irreducible tensor unit. We are interested in the case where there is a generator with intrinsic dimension $\geq 2$. No assumption on the existence of a braided symmetry or on amenability is made.

The tensor $\mathrm{C}^{*}$-categories $\mathcal{M}$ arising from subfactors have as objects the tensor powers of an irreducible selfconjugate object $x$, a property expressed in terms of an intertwiner $R \in(\iota, x \otimes x)$. The tensor *-subcategory generated by $R$ is a TemperleyLieb category, admitting an embedding if $d(x) \geq 2$. All such embeddings may be classified; they correspond to suitable compact quantum groups $A_{o}(F)$ of Wang and Van Daele. In particular, if $F \in M_{2}(\mathbb{C})$ these quantum groups are the quantum $\mathrm{SU}(2)$ groups of Woronowicz for deformation parameters uniquely determined by the dimension and the reality character of $x$. A similar result holds if the objects of $\mathcal{M}$ are the semigroup with unit generated by an object $x$ and its conjugate $\bar{x}$, the quantum groups involved are certain $A_{u}(F)$.

These remarks show that although tensor $C^{*}$-categories with conjugates cannot be embedded generically, they may contain an embeddable subcategory with conjugates.

Abstracting from the above, we start from two tensor $\mathrm{C}^{*}$-categories with conjugates $\mathcal{A}$ and $\mathcal{M}$, a tensor ${ }^{*}$-functor $\tau: \mathcal{A} \rightarrow$ Hilb and a quasi-tensor functor $\mu: \mathcal{A} \rightarrow \mathcal{M}$. We may suppose that the objects of $\mathcal{M}$ are tensor products of objects in the image of $\mu$. By [50], $\tau$ determines a compact quantum group $G_{\tau}$. We showed in [34] that the pair $\mu, \tau$ canonically defines an ergodic action of $G_{\tau}$ on a $\mathrm{C}^{*}$-algebra $\mathcal{C}$. When $\mu$ is the functor restricting a representation to a subgroup $K$ of $G_{\tau}$, this action reduces to the translation action on the quantum quotient space $K \backslash G_{\tau}$.

If $\mathcal{M}$ is not embeddable, the associated ergodic action cannot correspond to a true quantum subgroup [33]. Borrowing a notion due to Mackey [24], we may talk of a virtual subgroup.

The notions quasi-tensor functor and relaxed tensor functor are recalled in Section 2, this extra generality is motivated by their role in the duality theorem for ergodic actions of compact quantum groups, where they arise as spectral functors. (The spectral functor of an ergodic action maps a representation to the corresponding spectral space, thus, in particular, it takes values in the category of Hilbert spaces [34].) Note that, unlike relaxed tensor functors, quasi-tensor functors may take a non-zero object to the zero object.

One of the aims of this paper is to describe $\mathcal{M}$ as a category of representations of the virtual subgroup. To handle the non-embeddable case, we introduce the notion of a representation of a compact quantum group on a Hilbert bimodule over an ergodic 
$\mathrm{C}^{*}$-algebra. This is the noncommutative analogue of the bimodule of continuous sections of an equivariant Hermitian bundle over a compact homogeneous space. We show that these bimodule representations form a tensor $\mathrm{C}^{*}$-category with irreducible tensor unit, Theorem 3.1.

Given an object of $\mathcal{M}$, we construct a Hilbert bimodule representation of $G_{\tau}$, that we regard as a representation induced from the virtual subgroup, as the associated bimodule generalizes the bimodule of sections of the equivariant vector bundle induced from a representation of a subgroup. As a right module, the induced module turns out to be finite projective and, if $\mu$ is tensorial, even free and finitely generated, a result generalizing Swan's theorem to a noncommutative framework.

We show that the bimodule construction yields a full and faithful tensor *-functor, the induction functor, from $\mathcal{M}$ to the category $\operatorname{Bimod}_{\alpha}\left(G_{\tau}\right)$ of Hilbert bimodule representations of $G_{\tau}$. Note that this tensoriality property holds despite the fact that $\mu$ was only assumed to be quasi-tensor, Theorems 6.2 and 6.4.

In particular, if $\mathcal{M}$ is generated as a tensor $\mathrm{C}^{*}$-category by a real or pseudoreal object $x$ of $d(x) \geq 2$, then it may be identified with a category of bimodule representations for any one of a class of compact quantum groups $A_{o}(F)$. A similar result holds if $x$ is not selfconjugate, Theorems 6.5 and 6.6.

We then use these abstract results to investigate the case where $\mathcal{M}$ is an extension of the representation category of a compact Lie group $G$. We show that if the associated ergodic $C^{*}$-algebra $\mathcal{C}$ yields a finite type I von Neumann algebra after completion in the GNS-representation of the unique invariant trace, then $\mathcal{M}$ admits an embedding functor. In fact, we construct an explicit full tensor functor to the representation category of a closed subgroup of $G$, Theorem 6.7.

This last result shows that the question of the existence of an embedding into the Hilbert spaces is related to the open problem posed in [15] and mentioned in the abstract on the existence of ergodic actions of classical compact Lie groups on non-type- $I$ von Neumann algebras. A negative answer for $G$ would imply that the associated ergodic von Neumann algebra is of finite type I and hence that $\mathcal{M}$ is embeddable.

This is known for SU(2) [46]. This negative result shows that if the objects of $\mathcal{M}$ are generated by a single pseudoreal object of intrinsic dimension $2, \mathcal{M}$ can be embedded, and admits a full and faithful tensor *-functor to the category of representations of a closed subgroup of SU(2), Corollary 6.9. We would like to point out the analogy of this result with the well-known classification of subfactors of index 4 in terms of closed subgroups of SU(2).

The notion of full bimodule representation plays a role in this paper. An object of $\operatorname{Bimod}_{\alpha}(G)$ is full if every fixed vector for the action is central (see Section 5). This guarantees that the left module structure is naturally compatible with that of right module representation. We show that the induced bimodule representations are full (Propositions 8.9 and 9.4) and use this to show the embedding result. Furthermore we use this property to show that certain ergodic actions cannot arise from a pair of tensor functors $\mu, \tau$. We show for example that neither the adjoint action of a 
non-trivial irreducible representation of SU(2) nor those with full spectrum and low multiplicity can arise, Section 11.

Here we have interpreted induction in terms of bimodule representations. However we may also induce from representations of the virtual subgroup to Hilbert space representations of $G_{\tau}$, except that such representations need no longer be finitedimensional. However after completing our categories under infinite direct sums, we show that induction and restriction are a pair of adjoint functors, Theorem 10.1.

In conclusion, we draw the reader's attention to an incomplete list of papers where related results may be found, although some from different perspectives, [1], [5], [7], [9], [13], [16], [21], [26], [27], [31], [33], [42], [44].

The paper is organized as follows. Section 2 establishes notation and recalls results that we shall need. In Section 3, we explain the notion of a representation of a compact quantum group on a Hilbert $\mathrm{C}^{*}$-bimodule and we introduce the tensor $C^{*}$-category $\operatorname{Bimod}_{\alpha}(G)$. In Section 4 we review Mackey's induced representation and Frobenius reciprocity from the standpoint of bimodule representations. In the next section, we introduce the notion of full bimodule representation and discuss the example of quantum quotients. In Section 6 we illustrate the main ideas and results of this paper. Sections 7-9 are dedicated to the induction functor into the tensor category of Hilbert $C^{*}$-bimodules. In Section 7 we give the algebraic construction of bimodule and introduce an inner product starting from a pair $(\tau, \mu)$ and show positivity of the inner product when $\tau$ is tensorial in Section 8 , leading to the Hilbert $\mathrm{C}^{*}$-bimodule representation of the compact quantum group $G_{\tau}$ associated with $\tau$. In Section 9 we show that there is a unique extension of the induction functor to a tensor functor. In Section 10 we show that if we instead define an induction functor taking values in the category of unitary representations of $G_{\tau}$ on Hilbert spaces, then $(\mu$, Ind) is an adjoint pair of functors. Sections 11 and 12 are dedicated to the analysis of ergodic actions of compact groups. In Section 11 we classify full bimodule representations of compact groups on finite type I von Neumann algebras and use the classification in the following section to derive results on the problem of embedding into Hilbert spaces. A few computations in an appendix conclude the paper.

\section{Notation and preliminaries}

2.1. Tensor $\mathbf{C}^{*}$-categories with conjugates. We shall work with tensor $\mathrm{C}^{*}$-categories defined as in [11]. By MacLane's theorem [25], we may and shall assume that the tensor product is strictly associative. The tensor product between objects $u$ and $v$ will be denoted by $u \otimes v$ and between arrows $S$ and $T$ by $S \otimes T$. The $n$-th tensor power of an object $u$ will be denoted by $u^{n}$. The tensor unit, denoted by $\iota$, will always be assumed irreducible:

$$
(\iota, \iota)=\mathbb{C}
$$


An object $\bar{u}$ of a tensor $\mathrm{C}^{*}$-category $\mathcal{A}$ is a conjugate of $u$ if there are arrows

$$
R \in(\iota, \bar{u} \otimes u), \quad \bar{R} \in(\iota, u \otimes \bar{u})
$$

satisfying the conjugate equations

$$
\bar{R}^{*} \otimes 1_{u} \circ 1_{u} \otimes R=1_{u}, \quad R^{*} \otimes 1_{\bar{u}} \circ 1_{\bar{u}} \otimes \bar{R}=1_{\bar{u}} .
$$

If $R, \bar{R}$ is a solution of the conjugate equations for $u$, any other solution is of the form $X^{*-1} \otimes 1_{u} \circ R$ and $1_{u} \otimes X \circ \bar{R}$, where $X \in(\bar{u}, \tilde{u})$ is an invertible intertwiner. $\bar{R}$ is uniquely determined by $R$.

We will always take $1_{\iota}$ as the solution of the conjugate equations for $\iota$.

An object $u$ is called real or pseudoreal if we may choose $\bar{u}=u$ (i.e., $u$ selfconjugate), and a solution of the form $\bar{R}=R$ or $\bar{R}=-R$ respectively.

We shall say that $\mathcal{A}$ has conjugates if every object has a conjugate. In this case, every object is a direct sum of minimal projections. A solution of the conjugate equations $(R, \bar{R})$ is said to be standard if

$$
R^{*} \circ 1_{\bar{u}} \otimes Y \circ R=\bar{R}^{*} \circ Y \otimes 1_{\bar{u}} \circ \bar{R}, \quad Y \in(u, u) .
$$

The $X \in(\bar{u}, \tilde{u})$ taking one standard solutions to another is unitary. The intrinsic dimension of an object is defined as $d(u)=\|R\|^{2}$, where $R$ is part of a standard solution. Equivalently, $d(u)$ is the minimal value of $\|R\|\|\bar{R}\|$ for all solutions. We refer to [22] for details.

Fix objects $u, v$ of $\mathcal{A}$ and pick a solution $R_{u}, \bar{R}_{u}$ and $R_{v}, \bar{R}_{v}$ of the conjugate equations for $u$ and $v$ respectively, and define the associated antilinear map,

$$
A \in(v, u) \rightarrow A^{\bullet}:=R_{v}^{*} \otimes 1_{\bar{u}} \circ 1_{\bar{v}} \otimes A^{*} \otimes 1_{\bar{u}} \circ 1_{\bar{v}} \otimes \bar{R}_{u}, \quad \in(\bar{v}, \bar{u}) .
$$

This map depends on the choice of conjugates: changing the solution of the conjugate equations using invertibles $X \in(\bar{u}, \tilde{u})$ and $Y \in(\bar{v}, \tilde{v}), A^{\bullet}$ becomes $X \circ A^{\bullet} \circ Y^{-1}$.

We stress that the notation $R_{u}$ refers to a particular solution of the conjugate equations for $u$ but does not necessarily imply a choice for each object $u$.

Example. In the category Hilb any solution of the conjugate equations for a finitedimensional Hilbert space $H$ is of the form

$$
R=\sum_{h} \phi_{h} \otimes j^{-1} \phi_{h}, \quad \bar{R}=\sum_{k} \psi_{k} \otimes j \psi_{k},
$$

where $j$ is an antilinear invertible map to another Hilbert space $\bar{H}$ and $\left(\psi_{k}\right)$ and $\left(\phi_{h}\right)$ are orthonormal bases of $H$ and $\bar{H}$ respectively. We shall use the notation $j_{H}$ to emphasize that $j$ refers to the object $H$. For $A \in(H, K)$, the associated $\bullet$ is given by $A^{\bullet}=j_{K} A j_{H}^{-1}$. In particular, $\psi^{\bullet}=j_{H} \psi$ for $\psi \in H$. 
If $\left(R_{u}, \bar{R}_{u}\right),\left(R_{v}, \bar{R}_{v}\right)$ are solutions for $u$ and $v$ respectively, $R_{u \otimes v}:=1_{\bar{v}} \otimes R_{u} \otimes$ $1_{v} \circ R_{v}$ and $\bar{R}_{u \otimes v}:=1_{u} \otimes \bar{R}_{v} \otimes 1_{\bar{u}} \circ \bar{R}_{u}$ is a solution for $u \otimes v$, called the tensor product solution. Similarly, $R_{\bar{u}}:=\bar{R}_{u}$ and $\bar{R}_{\bar{u}}:=R_{u}$ the solution for $\bar{u}$, called the conjugate solution.

The main properties of $\bullet$ are the following:

$$
(A \circ B)^{\bullet}=A^{\bullet} \circ B^{\bullet}, \quad(A \otimes B)^{\bullet}=B^{\bullet} \otimes A^{\bullet}
$$

for the tensor product solution.

2.2. Quasitensor functors. Although all tensor categories may be assumed to be strict, it is well known that one may meet functors preserving the tensor structure only up to a natural equivalence. Here we need the notion of quasi-tensor functor whose definition we recall. $\mathrm{A}^{*}$-functor $\mu: \mathcal{A} \rightarrow \mathcal{M}$ is called quasi-tensor if there are isometries $\tilde{\mu}_{u, v} \in\left(\mu_{u} \otimes \mu_{v}, \mu_{u \otimes v}\right)$ such that

$$
\begin{gathered}
\mu_{\iota}=\iota, \\
\tilde{\mu}_{u, \iota}=\tilde{\mu}_{\iota, u}=1_{\mu_{u}}, \\
\tilde{\mu}_{u, v \otimes w}^{*} \circ \tilde{\mu}_{u \otimes v, w}=1_{\mu_{u}} \otimes \tilde{\mu}_{v, w} \circ \tilde{\mu}_{u, v}^{*} \otimes 1_{\mu_{w}}
\end{gathered}
$$

and natural in $u, v$,

$$
\mu(S \otimes T) \circ \tilde{\mu}_{u, v}=\tilde{\mu}_{u^{\prime}, v^{\prime}} \circ \mu(S) \otimes \mu(T),
$$

for objects $u, v, w, u^{\prime}, v^{\prime}$ of $\mathcal{A}$ and arrows $S \in\left(u, u^{\prime}\right), T \in\left(v, v^{\prime}\right)$. The above definition was given in [34] in a different form, in connection with the study of ergodic actions of compact quantum groups on unital $\mathrm{C}^{*}$-algebras. The equivalence with the above definition was shown in [35]. If all the isometries $\tilde{\mu}_{u, v}$ are unitary, $(\mu, \tilde{\mu})$ will be called a relaxed tensor functor. In particular, a strict tensor functor, or simply a tensor functor, is a quasi-tensor functor with $\tilde{\mu}_{u, v}:=1_{\mu_{u} \otimes \mu_{v}}$ for all objects $u, v$. Note that a quasi-tensor functor may take a non-zero object to the zero object. Examples arise from ergodic actions (cf. also Section 2.4).

Notice that, since we are dealing with isometries, (2.3) implies the associativity property,

$$
\tilde{\mu}_{u \otimes v, w} \circ \tilde{\mu}_{u, v} \otimes 1_{\mu_{w}}=\tilde{\mu}_{u, v \otimes w} \circ 1_{\mu_{u}} \otimes \tilde{\mu}_{v, w} .
$$

Hence both sides of this equation define the same intertwiner $\tilde{\mu}_{u, v, w} \in\left(\mu_{u} \otimes \mu_{v} \otimes\right.$ $\left.\mu_{w}, \mu_{u \otimes v \otimes w}\right)$. Iterating, we get, for any finite sequence $\underline{u}=\left(u_{1}, \ldots, u_{n}\right)$ of objects of $\mathcal{A}$, with $n \geq 2$, an unambiguous arrow

$$
\tilde{\mu}_{u_{1}, \ldots, u_{n}} \in\left(\mu_{u_{1}} \otimes \cdots \otimes \mu_{u_{n}}, \mu_{u_{1} \otimes \cdots \otimes u_{n}}\right) .
$$

We set $\tilde{\mu}_{u}=1_{\mu_{u}}$ for a sequence of length 1 . $\tilde{\mu}$ is a natural transformation, i.e., for $S_{i} \in\left(u_{i}, v_{i}\right)$,

$\mu\left(S_{1} \otimes S_{2} \otimes \cdots \otimes S_{n}\right) \circ \tilde{\mu}_{u_{1}, u_{2}, \ldots, u_{n}}=\tilde{\mu}_{v_{1}, v_{2}, \ldots, v_{n}} \circ \mu\left(S_{1}\right) \otimes \mu\left(S_{2}\right) \otimes \cdots \otimes \mu\left(S_{n}\right)$. 
We remark however that (2.3) is stronger than associativity. It also implies a categorical analogue of Popa's commuting square condition in the theory of subfactors, see [34] and references there. If $\mathcal{A}$ has conjugates, so do objects in the image of $\mu$. In detail, if $(\mu, \tilde{\mu}): \mathcal{A} \rightarrow \mathcal{M}$ is a quasi-tensor functor and if $(R, \bar{R})$ is a solution of the conjugate equations for $u$ and $\bar{u}$ in $\mathcal{A}$ then $\widehat{R}:=\tilde{\mu}_{\bar{u}, u}^{*} \circ \mu(R), \hat{\bar{R}}:=\tilde{\mu}_{\bar{u}, u}^{*} \circ \mu(\bar{R})$ is a solution of the conjugate equations for $\mu_{u}$ in $\mathcal{M}$, called the image solution. In particular, $d\left(\mu_{u}\right) \leq d(u)$.

Image solutions $\hat{R}_{u}, \hat{\bar{R}}_{u}$ and $\hat{R}_{v}, \hat{\bar{R}}_{v}$ associated to $u$ and $v$ define a map • on the arrow space $\left(\mu_{u}, \mu_{v}\right)$ in $\mathcal{M}$ and we have

$$
\mu(A)^{\bullet}=\mu\left(A^{\bullet}\right) .
$$

If $(\mu, \tilde{\mu}): \mathcal{A} \rightarrow \mathcal{M}$ and $(\nu, \tilde{v}): \mathcal{M} \rightarrow \mathcal{N}$ are quasi-tensor functors, the composition $v \mu$ becomes quasi-tensor with natural transformation $v\left(\tilde{\mu}_{u, v}\right) \circ \tilde{v}_{\mu_{u}, \mu_{v}}$ [35]. A composition $v \mu$ of two quasi-tensor functors will always be implicitly understood as a quasi-tensor functor with this natural transformation.

2.3. Ergodic $\mathrm{C}^{*}$-actions of compact groups. Let $\alpha: G \rightarrow \operatorname{Aut}(\mathcal{C})$ be a continuous ergodic action of a compact group $G$ on a unital $C^{*}$-algebra $\mathcal{C}$. The finiteness theorem for the noncommutative ergodic space $\mathcal{C}$ and the multiplicity bound theorem assert respectively that the unique $G$-invariant state of $\mathcal{C}$ is a trace, and that the multiplicity of an irreducible representation of $G$ in $\alpha$ is bounded above by its dimension. Furthermore, any von Neumann algebra with an ergodic action of a compact group is necessarily hyperfinite [15].

Recall that if $\beta$ is an automorphic action of a closed subgroup $K$ of $G$ on a von Neumann algebra $\mathcal{F}$, the induced von Neumann algebra is defined by:

$$
\begin{aligned}
\operatorname{Ind}(\mathcal{F}): & =\left\{f \in L^{\infty}(G, \mathcal{F}) \mid f(k g)=\beta_{k}(f(g)), k \in K, g \in G\right\} \\
& =\left(L^{\infty}(G) \bar{\otimes} \mathcal{F}\right)^{\lambda \otimes \beta},
\end{aligned}
$$

where $\lambda$ is left translation of $K$ on $L^{\infty}(G)$. If $\mathscr{F}$ is a $C^{*}$-algebra, the von Neumann tensor product $\bar{\otimes}$ is replaced by the minimal one, and $L^{\infty}$-functions by continuous ones. The induced algebra carries the induced action $\rho$ of $G$ given by right translation.

As recalled in [47], combining the above results with an imprimitivity theorem of Takesaki [43] for locally compact group actions on von Neumann algebras allows one to reduce the study of ergodic actions on von Neumann algebras to those on finite factors. Indeed, any ergodic action of a compact group $G$ on a von Neumann algebra $\mathcal{C}$ is induced by an action of a closed subgroup $K$ on a matrix algebra or on the hyperfinite $\mathrm{II}_{1}$ factor $R$.

Wassermann has shown the important result that $G=\mathrm{SU}(2)$ acts ergodically only on (finite) type I von Neumann algebras [46]. For more results in this direction see also [28]. It is not yet known whether there are any ergodic actions of compact classical Lie groups on the hyperfinite $\mathrm{II}_{1}$ factor $R$, a problem raised in [15]. 
2.4. Ergodic $\mathbf{C}^{*}$-actions of compact quantum groups. We refer to [51] for the general definition of a compact quantum group. If $G=(\mathcal{Q}, \Delta)$ is a compact quantum group, $\operatorname{Rep}(G)$ will denote the category of unitary finite-dimensional representations of $G$.

The theory of ergodic actions of compact quantum groups on unital $\mathrm{C}^{*}$-algebras has been initiated in [4], [37]. Recall from [37] that an action of $G$ on a unital $\mathrm{C}^{*}$-algebra $\mathcal{C}$ is a unital ${ }^{*}$-homomorphism

$$
\alpha: \varphi \rightarrow \varphi \otimes Q
$$

where $\otimes$ denotes the minimal tensor product of $\mathrm{C}^{*}$-algebras, such that $\alpha \otimes \iota \circ \alpha=$ $\iota \otimes \Delta \circ \alpha$ and with the property that $\alpha(\mathcal{C}) \mathbb{C} \otimes Q$ is dense in $\ell \otimes Q$. The action is called ergodic if $\ell^{\alpha}:=\{c \in \ell \mid \alpha(c)=c \otimes I\}=\mathbb{C}$. Recall that $\ell$ has a unique faithful state invariant under the action of $G$, but, unlike in the group case, is not a trace in general [49]; see also [45].

2.5. Spectrum, multiplicity maps, spectral functor and duality theorem. The spectrum of an action $\alpha$ of a compact quantum group $G$ on $\ell$, denoted by $\operatorname{sp}(\alpha)$, is the set of all unitary representations $u$ of $G$ for which there is a faithful linear map $T: H_{u} \rightarrow \mathcal{C}$ intertwining the representation $u$ with the action $\alpha$. This means that if $u_{i j}$ are the coefficients of $u$ in some orthonormal basis of $H$, there are linearly independent elements $c_{1}, \ldots, c_{d} \in \mathcal{C}$, with $d$ the dimension of $u$, such that $\alpha\left(c_{i}\right):=\sum_{j} c_{j} \otimes u_{j i}$. The linear span of all the $c_{i}$ 's, as $u$ varies in the spectrum, is a dense invariant *subalgebra of $\mathcal{C}$, denoted by $\ell_{\mathrm{sp}}$ [37].

Examples of ergodic actions are the quantum quotient spaces $C(K \backslash G)$ by a compact quantum subgroup $K$. As in the classical case, $C(K \backslash G)$ is the fixed point algebra under a suitable action of $K$ on the Hopf $C^{*}$-algebra of $G$, with action of $G$ given by restricting the coproduct. This action is usually called the translation action [45]; see also [33]. $C(K \backslash G)_{\text {sp }}$ is linearly spanned by the matrix coefficients $\left\{u_{k, \psi_{i}}\right\}$, where $u$ varies in the set of unitary finite-dimensional representations of $G, k$ in the set of fixed vectors for the restriction $u \uparrow K$ and $\left(\psi_{i}\right)$ is an orthonormal basis.

For any representation $u$, consider the space $L_{u}$ of all linear intertwiners $T$, not necessarily faithful, between $u$ and $\alpha$. $L_{u}$ becomes a Hilbert space with inner product $\langle S, T\rangle:=\sum_{i} T\left(\psi_{i}\right) S\left(\psi_{i}\right)^{*}$, with $\left(\psi_{i}\right)$ an orthonormal basis of $H_{u}$. For an irreducible $u, L_{u} \neq 0$ precisely when $v \in \operatorname{sp}(\alpha)$. The dimension of $L_{u}$ is the multiplicity of $u$ in $\alpha$. $L_{u}$ is known to be finite-dimensional if $\alpha$ is ergodic [4]. The complex conjugate vector space $\bar{L}_{u}$, is called the spectral space associated with $u$. For any $u \in \operatorname{Rep}(G)$, associate the map

$$
c_{u}: H_{u} \rightarrow \bar{L}_{u} \otimes \smile, \quad c_{u}(\psi):=\sum_{k} \bar{T}_{k} \otimes T_{k}(\psi),
$$

where $T_{k}$ is any orthonormal basis of $L_{u}$. Note that $c_{u}$ does not depend on the choice of orthonormal basis. The $c_{u}$ 's are called multiplicity maps in [34]. 
We can represent $c_{u}$ as a rectangular matrix whose $i$-th row is given by the multiplet $T_{i}=\left(T_{i}\left(\psi_{1}\right) \ldots T_{i}\left(\psi_{d}\right)\right)$ transforming like $u$ under $\alpha$.

The set of all coefficients $\left\{c_{i, j}^{u}=T_{i}\left(\psi_{j}\right), i, j\right\}$ forms a linear basis for the dense ${ }^{*}$-subalgebra $\ell_{\mathrm{sp}}$ as $u$ varies over a complete set of irreducible representations of $\operatorname{sp}(\alpha)$, [37], [2], [34].

The map $u \mapsto L_{u}$ can be extended to a functor $L: \operatorname{Rep}(G) \rightarrow$ Hilb from the category $\operatorname{Rep}(G)$ of unitary finite-dimensional representations of $G$ to the category of Hilbert spaces. If $A \in(u, v)$ and $T \in L_{v}$ then $T \circ A: H_{u} \rightarrow \ell$ lies in $L_{u} . L$ is a contravariant ${ }^{*}$-functor, hence it is convenient to pass to the dual Hilbert spaces, that we naturally identify with the spectral spaces $\bar{L}_{u}$. We thus get a covariant ${ }^{*}$-functor, $\bar{L}$, the spectral functor of the ergodic action. The spectral functor and the multiplicity maps are related by $\bar{L}_{A} \otimes I \circ c_{u}=c_{v} \circ A, A \in(u, v)$, for any $u, v \in \operatorname{Rep}(G)$.

For example, the spectral functor of a quantum quotient space $C(K \backslash G)$ maps the representation $u$ of $G$ to the Hilbert space of fixed vectors of the restriction $u \uparrow K$.

There is a natural isometric inclusion

$$
\widetilde{\bar{L}}_{u, v}: \bar{L}_{u} \otimes \bar{L}_{v} \rightarrow \bar{L}_{u \otimes v}
$$

identifying a simple tensor $\bar{S} \otimes \bar{T}$ with the complex conjugate of the element of $L_{\mathcal{u} \otimes v}$ defined by $\psi \otimes \phi \in H_{u} \otimes H_{v} \rightarrow S(\psi) T(\phi)$. It has been shown in [34] that $(\bar{L}, \widetilde{\bar{L}})$ is a quasi-tensor functor and that $(\mathcal{C}, \alpha)$ may be reconstructed almost entirely from $\bar{L}$. In detail, $\bar{L}$ keeps complete information on the dense ${ }^{*}$-subalgebra $\mathcal{C}_{\text {sp }}$, its maximal $\mathrm{C}^{*}$-norm and the restricted action $\alpha \uparrow \boldsymbol{\varphi}_{\mathrm{sp}}$. (However, $\bar{L}$ does not keep track of the original $\mathrm{C}^{*}$-norm of $\mathcal{C}$, a feature already present in Woronowicz' version of TannakaKrein duality [50].) Moreover, any quasi-tensor functor $(\mu, \tilde{\mu}): \operatorname{Rep}(G) \rightarrow$ Hilb is the spectral functor of an ergodic action of $G$ on a unital $C^{*}$-algebra.

For completeness, we recall that the spectral functor is a relaxed tensor functor if and only if the quantum multiplicity of every irreducible equals its quantum dimension. We refer to [2] for the notion of quantum multiplicity and to [34] for the proof of this fact. In the group case, this means that the multiplicity of every irreducible equals its dimension.

Note that the finiteness theorem fails for compact quantum groups, as the Haar measure is not a trace in general. On the other hand, the multiplicity bound theorem holds, provided multiplicity and dimension are replaced by their noncommutative analogues [4], [2].

2.6. Hilbert modules and Hilbert bimodules over $\mathbf{C} *$-algebras. We refer to [3], [20] for the definition of a (right) Hilbert module $X$ over a $\mathrm{C}^{*}$-algebra $\ell$. The $\ell$ -

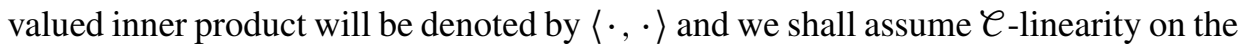
right. We recall in particular that if $\ell=\mathbb{C}, X$ is just a Hilbert space. Any $\mathrm{C}^{*}$-algebra $\zeta$ gives rise to the Hilbert module, $X=\ell$, with inner product $\left\langle c, c^{\prime}\right\rangle:=c^{*} c^{\prime}$. This is usually called the trivial Hilbert module. More generally, if $H$ is a Hilbert space, we may form the algebraic tensor product $H \odot \mathcal{C}$, which is a pre-Hilbert module with 
the obvious module structure and inner product

$$
\left\langle\psi \otimes c, \psi^{\prime} \otimes c^{\prime}\right\rangle=\left\langle\psi, \psi^{\prime}\right\rangle c^{*} c^{\prime} .
$$

The completion will be denoted by $H \otimes \mathcal{C}$. We shall only consider Hilbert modules over unital $\mathrm{C}^{*}$-algebras. A Hilbert $\varphi$-module $X$ is called full if the inner products $\left\langle x, x^{\prime}\right\rangle$ span a dense subspace of $C$.

If $X$ and $X^{\prime}$ are Hilbert modules over $\mathcal{C}, \mathscr{L}_{\mathcal{C}}\left(X, X^{\prime}\right)$ denotes the Banach space of bounded adjointable maps from $X$ to $X^{\prime}$.

A Hilbert module $X$ over $C$ will be called a Hilbert bimodule if there is a left action of $\mathcal{C}$ on $X$ given by a unital ${ }^{*}$-homomorphism $\mathcal{C} \rightarrow \mathscr{L} e(X, X)$. For example, the trivial module becomes a Hilbert bimodule in the obvious way. If $X^{\prime}$ is another Hilbert bimodule over $\ell_{e} \mathscr{L}_{e}\left(X, X^{\prime}\right)$ denotes the set of elements $T \in \mathscr{L}_{e}\left(X, X^{\prime}\right)$ commuting with the left actions.

The great advantage of Hilbert bimodules versus Hilbert modules is that we may form tensor products, $X \otimes_{e} Y$ see [3], [6]. The category with objects Hilbert bimod-

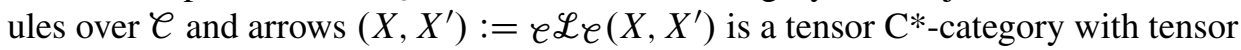
unit given by the trivial Hilbert bimodule.

\section{Bimodule representations of compact quantum groups}

In this section we define unitary representations of compact quantum groups on Hilbert modules or Hilbert bimodules over unital $\mathrm{C}^{*}$-algebras. These representations may be regarded as the noncommutative analogues of the $G$-equivariant Hermitian bundles over compact spaces introduced by Segal [40], where $G$ is a compact group.

In the following general definition we shall not assume our modules to be finite projective (this would correspond to local triviality in the commutative case, by Swan's theorem [41]), even though we shall eventually be interested in finite projective Hilbert modules.

Let us fix an action $(\mathcal{C}, \alpha)$ of a compact quantum group $G=(\mathcal{Q}, \Delta)$ on a uni-

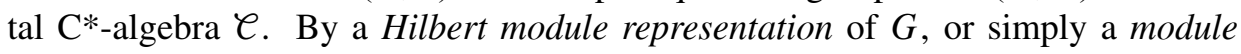
representation, we mean a $\mathbb{C}$-linear map

$$
v: X_{v} \rightarrow X_{v} \otimes \mathbb{Q}
$$

where $X_{v}$ is a Hilbert $\mathcal{C}$-module, $\mathcal{Q}$ is regarded as the trivial Hilbert $\mathcal{Q}$-module and $X_{v} \otimes \mathcal{Q}$ denotes the exterior tensor product of Hilbert modules, a Hilbert module over $\mathcal{Q} \otimes$, see [3] for details, such that

$$
\begin{gathered}
\left\langle v(x), v\left(x^{\prime}\right)\right\rangle_{e \otimes Q}=\alpha\left(\left\langle x, x^{\prime}\right\rangle \mathcal{e}\right), \quad x, x^{\prime} \in X_{v}, \\
v(x c)=v(x) \alpha(c), \quad x \in X_{v}, c \in \mathcal{C}, \\
v \otimes 1_{\mathcal{Q}} \circ v=1_{X_{v}} \otimes \Delta \circ v, \\
v\left(X_{v}\right) 1_{X_{v}} \otimes \mathcal{Q} \text { is dense in } X_{v} \otimes \mathcal{Q} .
\end{gathered}
$$


The simplest example of a module representation is the trivial representation, $v=\alpha$ on the trivial Hilbert $\mathcal{\ell}$-module. It will be denoted by $\iota$.

Note that if $\mathcal{C}=\mathbb{C}$ this definition reduces to the notion of a strongly continuous unitary representation of a compact quantum group on a Hilbert space; see [1], [51].

On the other hand, as mentioned at the beginning of the section, if $\mathcal{C}$ is commutative and $G$ is a compact group, this notion reduces to that of a $G$-equivariant Hermitian bundle, the equivariance property being expressed by (3.2).

One can form the $\mathrm{C}^{*}$-category $\operatorname{Mod}_{\alpha}(G)$ with objects the module representations of $G$ and arrows

$$
\left(v, v^{\prime}\right):=\left\{T \in \mathscr{L}_{\mathcal{C}}\left(X_{v}, X_{v^{\prime}}\right) \mid v^{\prime} \circ T=T \otimes 1_{\mathcal{Q}} \circ v\right\} .
$$

Note that $(\iota, \iota)$ can be identified with the fixed point algebra $\ell^{\alpha}$.

We are interested in module representations of a compact quantum group where $X_{v}$ is a Hilbert bimodule. $v$ will be called a (Hilbert) bimodule representation of $G$ if in addition to (3.1)-(3.4),

$$
v(c x)=\alpha(c) v(x), \quad c \in \mathcal{C}, x \in X_{v} .
$$

As an example, the trivial representation $\iota$ is a bimodule representation, that we shall denote by the same symbol.

We denote by $\operatorname{Bimod}_{\alpha}(G)$ the category with objects Hilbert bimodule representations and arrows $\left(v, v^{\prime}\right)$ between two of them the space of intertwining operators in $\operatorname{Mod}_{\alpha}(G)$ which in addition intertwine the left actions of $\mathcal{C}$. If $u, v$ are two objects of $\operatorname{Bimod}_{\alpha}(G)$ we define, for $x \in X_{u}, y \in X_{v}$, the tensor product bimodule representation $u \otimes v$ by

$$
u \otimes v(x \otimes y)=u(x)_{13} v(y)_{23},
$$

an element of $X_{u} \otimes e X_{v} \otimes Q$ Q (3.2) and (3.5) show that $u \otimes v$ is well defined on the algebraic bimodule tensor product $X_{u} \odot_{\mathcal{e}} X_{v}$ and that (3.1), (3.2), (3.3) and (3.5) hold. The validity of (3.1) implies that $u \otimes v$ extends uniquely to a bounded $\mathbb{C}$-linear map

$$
u \otimes v: X_{u} \otimes e X_{v} \rightarrow X_{u} \otimes_{e} X_{v} \otimes \mathcal{Q},
$$

and the above equations still hold whereas (3.4) holds by construction. The tensor product of two intertwiners is now well defined and intertwines the tensor product representations. Note that if $v$ is an object of $\operatorname{Bimod}_{\alpha}(G), v \otimes \iota$ and $\iota \otimes v$ are equivalent to $v$ in $\operatorname{Bimod}_{\alpha}(G)$. This leads to the following result.

Theorem 3.1. The category $\operatorname{Bimod}_{\alpha}(G)$ with objects Hilbert bimodule representations of $G$ and arrows the bimodule intertwining operators is a tensor $C^{*}$-category. The tensor unit is the trivial representation $\iota$ and $(\iota, \iota)=\{c \in Z(\mathcal{C}) \mid \alpha(c)=c \otimes I\}$, the set of central fixed points. There is an obvious faithful ${ }^{*}$-functor $\operatorname{Bimod}_{\alpha}(G) \rightarrow$ $\operatorname{Mod}_{\alpha}(G)$.

We shall only consider ergodic actions, hence $(\iota, \iota)=\mathbb{C}$. 


\section{The induced $C^{*}$-bimodules for compact groups}

We recall Mackey's definition [23] of a representation induced from a closed subgroup of a compact group and the Frobenius reciprocity theorem in the form later generalized to tensor $\mathrm{C}^{*}$-categories.

Our discussion has points in common with [39]. The main point is that we shall pass from Hilbert space representations to Hilbert bimodule representations. The module approach to induction is particularly convenient in the compact case as it provides finite-dimensional objects by Swan's theorem [41] and moreover the induction functor has good tensorial properties (cf. Theorem 4.1).

4.1. Mackey's induced representation and Frobenius reciprocity. Let $K$ be a closed subgroup of a compact group $G$ and $v$ a (unitary, finite-dimensional) representation of $K$ on the Hilbert space $H_{v}$. Mackey's induced representation $\operatorname{Ind}(v)$ is defined as right translation by elements of $G$ on the Hilbert space of $L^{2}$ functions $\zeta$ on $G$ with values in $H_{v}$ satisfying

$$
\zeta(k g)=v(k) \zeta(g), \quad k \in K, g \in G,
$$

where the inner product $\left\langle\zeta, \zeta^{\prime}\right\rangle=\int_{K \backslash G}\left\langle\zeta(g), \zeta^{\prime}(g)\right\rangle d \mu$ involves the unique normalized $G$-invariant measure $\mu$ on $K \backslash G$.

The main result is the Frobenius reciprocity theorem, asserting that there is an explicit linear isomorphism from the intertwining space $\left(u \uparrow_{K}, v\right)$ to $(u$, $\operatorname{Ind}(v))$, taking an intertwiner $S$ to the intertwiner $T$, where $T(\psi)(g)=S(u(g) \psi)$. The Frobenius isomorphism is natural in $u$ and $v$, and hence makes restriction and induction into a pair of adjoint functors. (In Section 10, we will briefly recall the notion of an adjoint pair of functors. For details we refer the reader to MacLane's book [25].) Consequently, the spectrum of the induced representation $\operatorname{Ind}(v)$ is the set of all irreducible $G$-representations $u$ for which $\left(u \uparrow_{K}, v\right)$ is nonzero. Another consequence is that any irreducible representation $v$ of $K$, and hence any $v$, is a subrepresentation of some restriction to $K$ of a representation $u$ of $G$. Finally, the explicit form of the isomorphism shows that if $T \in(u$, Ind $(v))$ all the functions $T(\psi)$ are continuous. This last remark leads to the next step.

4.2. Replacing Hilbert spaces with Hilbert bimodules. Since we do not loose any information on the arrows, we may replace Hilbert spaces with Hilbert bimodules. More precisely, we pass from the Hilbert space of the induced representation to the space $C_{v}$ of continuous $H_{v}$-valued functions $\zeta$ as above, which is a bimodule over the commutative $\mathrm{C}^{*}$-algebra $C(K \backslash G)$ of continuous functions on the quotient space in the obvious way. $C_{v}$ has an inner product given by pointwise evaluation of the inner product of $H_{v}$,

$$
\left\langle\zeta, \zeta^{\prime}\right\rangle(g):=\left\langle\zeta(g), \zeta^{\prime}(g)\right\rangle
$$

This inner product is constant on each left coset $K g$ as $v$ is unitary, and $C_{v}$ becomes a Hilbert bimodule over $C(K \backslash G)$. Hence $\operatorname{Ind}(v)$ becomes a Hilbert $C(K \backslash G)$-bimodule 
representation of $G$ in the sense of the previous section, where $\alpha$ is given by right translation by elements of $G$ on the quotient space. Note that $C_{v}$ is the bimodule of continuous sections of the classical equivariant vector bundle induced from $v$.

4.3. The induction functor and Swan's theorem. We thus have a ${ }^{*}$-functor

$$
\text { Ind: } \operatorname{Rep}(K) \rightarrow \operatorname{Bimod}_{\alpha}(G),
$$

taking an object $v$ of $\operatorname{Rep}(K)$ to $\operatorname{Ind}(v)$ and an arrow $T \in\left(v, v^{\prime}\right)$ to the arrow $\operatorname{Ind}(T) \in\left(\operatorname{Ind}(v), \operatorname{Ind}\left(v^{\prime}\right)\right)$ defined by $\operatorname{Ind}(T) \xi(g)=T \xi(g)$. We shall refer to Ind as the induction functor.

If $u \uparrow_{K}$ is the restriction of a representation $u$ of $G$ to $K$, there is a natural faithful bimodule map,

$$
U: C_{u \uparrow_{K}} \rightarrow H_{u} \otimes C(K \backslash G), \quad U \zeta(g)=u\left(g^{-1}\right) \zeta(g) .
$$

$U$ is invertible, and hence surjective, with inverse given by $U^{-1} \xi(g)=u(g) \xi(g)$. Hence $C_{u \uparrow_{K}}$ is free as a right (and left) module. Moreover, $U$ becomes unitary when $H_{u} \otimes C(K \backslash G)$ is regarded as a Hilbert bimodule. Note that the tensor product action $u \otimes \alpha$ of $G$ on $H_{u} \otimes C(K \backslash G)$ is a Hilbert bimodule representation of $G$ and $U$ becomes a unitary intertwiner from $\operatorname{Ind}\left(u \uparrow_{K}\right)$ to $u \otimes \alpha$ in $\operatorname{Bimod}_{\alpha}(G)$.

Let now $v$ be a generic unitary finite-dimensional representation of $K$, and consider a restricted representation $u \uparrow_{K}$ containing $v$ as a subrepresentation. An isometric intertwiner in $\left(v, u \uparrow_{K}\right)$ defines an isometric intertwiner in $\left(\operatorname{Ind}(v), \operatorname{Ind}\left(u \uparrow_{K}\right)\right)$ between the associated Hilbert bimodules representations, via the induction functor. Therefore $\operatorname{Ind}(v)$ is a subobject of $\operatorname{Ind}\left(u \uparrow_{K}\right)$. Moreover, composition with the map $U$ as above, gives rise to an isometry of Hilbert modules from $C_{v}$ to $H_{u} \otimes C(K \backslash G)$. Hence $C_{v}$ is a finite projective module. This is essentially Rieffel's proof [39] of Swan's theorem [41].

Taking into account the previous remark, we may conclude that bimodule representations of the form $u \otimes \alpha$ suffice to generate the category of all induced bimodule representations via subobjects. This viewpoint will play a role in the next sections.

On the other hand, the naturality of the Frobenius isomorphism shows that any element of $\left(\operatorname{Ind}(v), \operatorname{Ind}\left(v^{\prime}\right)\right)$ is of the form $\operatorname{Ind}(T)$ for a unique $T \in\left(v, v^{\prime}\right)$, and so Ind is a full functor.

4.4. Tensorial properties of the induction functor. We next analyse the behaviour of Ind under tensor products. We may consider the tensor product of Hilbert bimodules, $C_{u} \otimes C_{v}:=C_{u} \otimes C(K \backslash G) C_{v}$. There are obvious isometric inclusions of $G$-bimodule representations $C_{u} \otimes C_{v} \rightarrow C_{u \otimes v}$. These maps are in fact surjective, and hence unitary, as, given isometries $S \in\left(u,\left.u^{\prime}\right|_{K}\right)$ and $T \in\left(v, v^{\prime} \uparrow_{K}\right)$, module bases for $C_{u}$ and $C_{v}$ are given by the functions $\left(x_{i}:=g \rightarrow S^{*} u^{\prime}(g) \psi_{i}\right)$ and $\left(y_{j}:=g \rightarrow T^{*} v^{\prime}(g) \phi_{j}\right)$ respectively, where $\psi_{i}$ and $\phi_{j}$ are orthonormal bases of the 
Hilbert spaces of $u^{\prime}$ and $v^{\prime}$. Hence, as $u \otimes v$ is a subrepresentation of $\left(u^{\prime} \otimes v^{\prime}\right) \uparrow_{K}$, a module basis for $C_{u \otimes v}$ is given by the functions $g \rightarrow S^{*} \otimes T^{*} u^{\prime} \otimes v^{\prime}(g) \psi_{i} \otimes \phi_{j}=$ $x_{i}(g) \otimes y_{j}(g)$. Thus $C_{u \otimes v}$ can be naturally identified with $C_{u} \otimes C_{v}$. It follows that Ind is a relaxed tensor functor in the sense recalled in Sect. 2. We summarize this discussion in the following theorem, essentially a geometric form of the Frobenius reciprocity theorem.

Theorem 4.1. The induction functor Ind: $\operatorname{Rep}(K) \rightarrow \operatorname{Bimod}_{\alpha}(G)$ is a full and faithful ${ }^{*}$-functor into the bimodule representation category of $G$, where $\alpha$ is given by right translation of $G$ on $C(K \backslash G)$. For any $v \in \operatorname{Rep}(K)$, the $C(K \backslash G)$-bimodule $C_{v}$ of $\operatorname{Ind}(v)$ is finite projective. In particular, if $v$ is the restriction of a representation of $G, C_{v}$ is free. The natural unitaries $C_{u} \otimes C_{v} \rightarrow C_{u \otimes v}$ make Ind into a relaxed tensor functor.

\section{Full bimodule representations}

In this section we introduce a notion central to this paper, that of a full bimodule representation of a compact quantum group $G$. This is a compatibility condition between the left and right bimodule structure of a Hilbert $\mathrm{C}^{*}$-bimodule carrying a representation of $G$. As we shall see, in the classical case, triviality of the bimodule structure means that every induced bimodule representation is full. The importance of this notion is that all induced bimodule representations of compact quantum groups constructed in this paper are full.

In the noncommutative situation, $C(K \backslash G)$ with the translation action is replaced by a compact quantum group $G$ acting ergodically on a unital $\mathrm{C}^{*}$-algebra $\ell$. The action will be denoted by $\alpha$. It would be too restrictive to consider just quantum quotient spaces $K \backslash G$, as $G$ can act on far more noncommutative $\mathrm{C}^{*}$-algebras. Following Mackey, we may regard the ergodic action $(\mathcal{C}, \alpha)$ as arising from a virtual subgroup.

Restricting or inducing a representation now looses its strict meaning. What is left is the analogue of $\operatorname{Ind}\left(u \uparrow_{K}\right)$, which may be defined as acting on the free Hilbert module $H_{u} \otimes \mathcal{C}$. More precisely, it is easy to see that the map $u \otimes \alpha$ defined by

$$
u \otimes \alpha(\psi \otimes c):=u(\psi)_{13} \alpha(c)_{23} \in H_{u} \otimes \mathcal{Q} \otimes \mathbb{Q}, \quad \psi \in H_{u}, c \in \mathcal{C},
$$

is a Hilbert module representation of $G$ on $H_{u} \otimes \ell$.

Given $T \in(u, v)$, with $u, v \in \operatorname{Rep}(G)$, define $T \otimes 1 \varphi: H_{u} \otimes \smile \rightarrow H_{v} \otimes \smile$ by $T \otimes 1_{e}(\psi \otimes c)=T \psi \otimes c$ then $T \otimes 1 e \in(u \otimes \alpha, v \otimes \alpha)$ in $\operatorname{Mod}_{\alpha}(G)$.

Proposition 5.1. The map $\operatorname{Rep}(G) \rightarrow \operatorname{Mod}_{\alpha}(G)$, taking $u \rightarrow u \otimes \alpha$ and $T \in$ $(u, v) \rightarrow T \otimes 1$, is a faithful ${ }^{*}$-functor between $C^{*}$-categories.

The previous proposition is a very weak form of Theorem 4.1 and our aim is to generalize it to the noncommutative setting. This involves giving $H_{u} \otimes e_{\text {a left }}$ 
module structure making $u \otimes \alpha$ into a Hilbert bimodule $G$-representation for all $u$. Although classical induction corresponds to the simplest solution (trivial bimodule structure), in noncommutative case, it is not obvious how to select ergodic actions $(\mathcal{C}, \alpha)$ in such a way that the module representation $u \otimes \alpha$ can be completed to a bimodule representation. Even if this is the case, many left module structures will not be relevant, and we claim that a compatibility condition between left and right module structure is needed. This may already be seen for a compact group $G$ acting on a noncommutative $\mathrm{C}^{*}$-algebra $\mathcal{C}$.

In this case the most natural left module structure on $H_{u} \otimes \mathcal{C}$ is the obvious left multiplication by elements of $\mathcal{C}$. With this left $\mathcal{C}$-action, the right Hilbert module representation $u \otimes \alpha$ becomes a Hilbert bimodule representation.

However, an intertwiner between two such bimodule representations, being a bimodule map, must lie in $\mathcal{B}\left(H_{u}, H_{u^{\prime}}\right) \otimes Z(\mathcal{C})$, where $Z(\mathcal{C})$ is the centre of $\mathcal{C}$. Hence these intertwiners do not see the noncommutativity of $\mathcal{\ell}$, in contrast to the module intertwining spaces, where $(\iota, u \otimes \alpha)$ is the space of multiplets $\xi=\left(c_{1}, \ldots, c_{d}\right)$, with $c_{i} \in \mathcal{C}$ and $\alpha\left(c_{i}\right)=\sum_{j} c_{j} \otimes u_{j i}^{*}, d$ being the dimension of $u$. As $u$ varies over the irreducible spectral representations of $G$, the linear span of the corresponding $c_{i}$ 's is a dense invariant ${ }^{*}$-subalgebra, cf. Sect. 2.

Hence the natural left action on $H_{u} \otimes \mathcal{C}$ gives rise to a tensor category which does not allow one to reconstruct $\mathcal{C}$, but only its centre. It would be desirable to use instead a left $\mathcal{C}$-action on $H_{u} \otimes \mathcal{C}$ where all the module $G$-intertwiners become bimodule $G$-intertwiners. This leads us to the notion of full bimodule representation.

Definition. Let $G$ be a compact quantum group. A fixed vector $\xi$ for a module representation $v$ on $X_{v}$ is an element $\xi \in X_{v}$ such that $v(\xi)=\xi \otimes I$. The set of fixed vectors for $v$ is precisely the intertwining space $(\iota, v)$ in $\operatorname{Mod}_{\alpha}(G)$. A bimodule representation $v$ will be called full if every fixed vector $\xi$ for the underlying module $G$-representation is central: $\xi c=c \xi$ for $c \in \mathcal{C}$.

Note that the trivial representation is full since $\alpha$ is ergodic.

The next result shows that if $G$ is a group, classical induction is characterized among functors $u \rightarrow u \otimes \alpha$ from $\operatorname{Rep}(G)$ to $\operatorname{Mod}_{\alpha}(G)$ by the property that under the natural left action each $u \otimes \alpha$ becomes a full bimodule representation.

Proposition 5.2. Let $G$ be a compact group and $\alpha$ an ergodic action of $G$ on a unital $C^{*}$-algebra $\mathcal{C}$. Then the natural left $\mathcal{C}$-action turns $u \otimes \alpha$ into a full representation for all $u \in \operatorname{Rep}(G)$ if and only if $\mathcal{C}$ is commutative. In this case, $\mathcal{C}=C(K \backslash G)$ for a closed subgroup $K$, unique up to conjugation, where $\alpha$ acts by right translation, $\alpha_{g} f\left(g^{\prime}\right)=f\left(g^{\prime} g\right), f \in C(K \backslash G)$. Hence $u \otimes \alpha$ corresponds to the classical induced representation $\operatorname{Ind}\left(u \uparrow_{K}\right)$.

Proof. If $\mathcal{C}$ is commutative, $c \eta=\eta c$ for $c \in \mathcal{C}, \eta \in H_{u} \otimes \mathcal{C}$ and $u \in \operatorname{Rep}(G)$. Hence any module intertwiner between $u \otimes \alpha$ and $u^{\prime} \otimes \alpha$ is a bimodule intertwiner. 
In particular, $u \otimes \alpha$ is full for all $u$. Conversely, assume that all the $u \otimes \alpha$ are full. We have already seen that a fixed vector $\xi$ for $u \otimes \alpha$ has the form $\xi=\sum_{j} \psi_{j} \otimes c_{i}$ for an orthonormal basis $\left(\psi_{j}\right)$ of $H_{u}$ where $c_{j}$ transforms like the complex conjugate representation $u_{*}=\left(u_{i j}^{*}\right)$ under $\alpha$. Since $\xi$ is supposed central, the elements $c_{i}$ are central in $\zeta$. If $u$ varies in the spectrum of $\alpha$, we get a dense commutative ${ }^{*}$ subalgebra of $\ell$, hence $\ell$ is commutative. As is well known, when $\alpha$ is an ergodic action on a unital commutative $C^{*}$-algebra, the action is right translation on $C(K \backslash G)$ by elements of $G$ for a closed subgroup $K$ of $G$, unique up to conjugation. Hence $u \otimes \alpha$ can be identified with $\operatorname{Ind}\left(u \uparrow_{K}\right)$.

Of course, we expect that, requiring all $u \otimes \alpha$ to be full bimodule representations for some left module structure, selects a proper subclass of ergodic actions. This is indeed the case, as we shall see (cf. Sect. 6 and references there to later sections).

The situation becomes significantly worse if $G$ is a quantum group, where the natural left action on $H_{u} \otimes \mathcal{C}$ may not even lead to a bimodule representation structure on $u \otimes \alpha$.

Proposition 5.3. Let $G$ be a compact quantum group, $u \in \operatorname{Rep}(G)$ and $\alpha$ an ergodic action of $G$ on a unital $C^{*}$-algebra $\mathcal{C}$. Then $u \otimes \alpha$ is a bimodule representation for the natural left module structure if and only if all coefficients of the irreducibles in the spectrum of $\alpha$ commute with the coefficients of $u$.

Proof. $C$ is generated as a Banach space, by the entries of rectangular matrices $\left(c_{i, j}^{v}\right)$ transforming like irreducible $G$-representations

$$
\alpha\left(c_{i, j}^{v}\right)=\sum_{p} c_{i, p}^{v} \otimes v_{p, j}
$$

These entries are linearly independent [37], [2], [34] and the conclusion follows from (3.5) .

We next discuss examples of full bimodule representations arising from quantum quotients.

Examples from quantum quotients. Let $G=(\mathcal{Q}, \Delta)$ be a compact quantum group, and $K$ a quantum subgroup. $G$ acts on the quotient space $K \backslash G$ by right translation, given by restricting the coproduct $\Delta$ of $G$. One can consider the left $C(K \backslash G)$-action on $H_{u} \otimes C(K \backslash G)$ defined as follows. For $c \in C(K \backslash G)$, consider the element $\lambda_{u}(c) \in \mathscr{L}\left(H_{u}\right) \otimes \mathcal{Q}$ defined by

$$
\left\langle\psi_{i} \otimes I, \lambda_{u}(c) \psi_{j} \otimes I\right\rangle:=\sum_{h} u_{h i}^{*} c u_{h j}
$$

where $\left(\psi_{i}\right)$ is an orthonormal basis of $H_{u}$. It is easy to check that this element is independent of the choice of orthonormal basis. One could show directly that $\lambda_{u}(c) \in$ $\mathscr{L}\left(H_{u}\right) \otimes C(K \backslash G)$ and that $\lambda_{u}$ makes $u \otimes \alpha$ into a full bimodule representation. 
However, we refrain from giving complete details, as this will be proved in more generality in Sect. 8. We just verify that it is full. If $\xi:=\sum \psi_{j} \otimes c_{j} \in H_{u} \otimes C_{\text {is a }}$ fixed vector, i.e., $\Delta\left(c_{i}\right)=\sum_{j} c_{j} \otimes u_{j i}^{*}$, then

$$
c \xi=\sum_{k} \psi_{k} \otimes\left\langle\psi_{k} \otimes I, \lambda_{u}(c) \xi\right\rangle=\sum_{k, j, h} \psi_{k} \otimes u_{h k}^{*} c u_{h j} c_{j}
$$

whereas

$$
\xi c=\sum_{k} \psi_{k} \otimes c_{k} c
$$

hence we need to show that for every $c \in C(K \backslash G)$,

$$
\sum_{h, j} u_{h k}^{*} c u_{h j} c_{j}=c_{k} c
$$

for all $k$. On the other hand, for a quotient space, we can find a $K$-fixed vector $\eta$ of $H_{u}$ such that $c_{j}=u_{\eta, j}^{*}$, and the desired equality follows from the unitarity of $u$; see, e.g., Section 2 in [34].

We are thus left with the problem of finding full Hilbert bimodules that lead to an induction functor with good tensorial properties. We shall show that tensor $\mathrm{C}^{*}$ categories with conjugates provide a natural solution to this problem, as well as large classes of ergodic actions of compact quantum groups, among them the compact quantum quotient spaces. However, many more will be discussed in Section 6.

\section{Main results}

In this section we illustrate the main ideas and results. Proofs will be given in later sections.

We start with a pair of tensor $\mathrm{C}^{*}$-categories $\mathcal{A}$ and $\mathcal{M}$ related by a quasi-tensor functor $\mu: \mathcal{A} \rightarrow \mathcal{M}$. The category $\mathcal{A}$ is assumed to be embeddable into Hilbert spaces and we then pick a tensor functor $\tau: \mathcal{A} \rightarrow$ Hilb. We will assume that $\mathcal{A}$ has conjugates.

The simplest example is provided by a closed subgroup $K$ of a compact group $G$. We may choose $\tau: \operatorname{Rep}(G) \rightarrow$ Hilb the embedding functor and $\mu: \operatorname{Rep}(G) \rightarrow$ $\operatorname{Rep}(K)$ the tensor functor restricting a representation of $G$ to $K$.

Note however that this example has certain special features, like the fact that $\mathcal{M}=\operatorname{Rep}(K)$ is embeddable, or that $\mu$ is tensorial. In general, $\mathcal{M}$ is not assumed to be embeddable, and, as recalled in Section 2, a quasi-tensor functor $\mu$, unlike a relaxed tensor functor, may take a nonzero object to a zero object.

By Woronowicz duality, the embedding $\tau$ defines a compact quantum group $G_{\tau}$ such that every object $u \in \mathcal{A}$ has an associated representation $\hat{u} \in \operatorname{Rep}\left(G_{\tau}\right)$ on the Hilbert space $\tau_{u}$. The arrow spaces of $\operatorname{Rep}\left(G_{\tau}\right)$ are the images under $\tau$ of the arrow spaces of $\mathcal{A},(\hat{u}, \hat{v})=\tau((u, v))$. 
The pair $(\tau, \mu)$ determines canonically a unital $\mathrm{C}^{*}$-algebra, $\mathcal{\ell}$ and an ergodic action $\alpha$ of $G_{\tau}$ on $\mathcal{C}$. This fact has been shown in [34] in the special case where $\mu: \mathcal{A} \rightarrow \mathcal{M}$ is a tensor functor. To see that this holds in our more general setting consider the composed ${ }^{*}$-functor

$$
F: \mathcal{A} \stackrel{\mu}{\rightarrow} \mathcal{M} \rightarrow \text { Hilb, }
$$

where the second functor is the so called minimal functor $x \in \mathcal{M} \rightarrow(\iota, x) \in$ Hilb, which is quasi-tensor; see [34]. Since composition of quasi-tensor functors is quasi-tensor (cf. Section 2.2), so is $F$. Thus there is a unique quasi-tensor functor $(\nu, \tilde{v}): \operatorname{Rep}\left(G_{\tau}\right) \rightarrow$ Hilb such that the following diagram commutes:

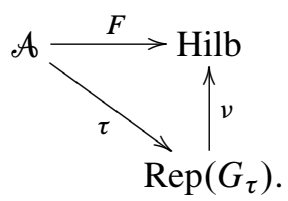

It follows that $(\nu, \tilde{v})$ is the spectral functor of an ergodic $\mathrm{C}^{*}$-action of $G_{\tau}$ (cf. Section 2.5), and this is $(\mathcal{C}, \alpha)$. The following simple remark clarifies matters.

Proposition 6.1. For a fixed $\tau: \mathcal{A} \rightarrow \operatorname{Hilb}$, let $(\mu, \tilde{\mu}): \mathcal{A} \rightarrow \mathcal{M},\left(\mu^{\prime}, \tilde{\mu}\right): \mathcal{A} \rightarrow$ $\mathcal{M}^{\prime}$ be a pair of quasi-tensor functors. If there is a full relaxed tensor functor $(\phi, \tilde{\phi}): \mathcal{M} \rightarrow \mathcal{M}^{\prime}$ such that $\phi \mu=\mu^{\prime}$, then the associated ergodic $C^{*}$-actions $(\mathcal{C}, \alpha)$ and $\left(\mathcal{C}^{\prime}, \alpha^{\prime}\right)$ are conjugate.

Proof. By 8.4 in [34] two ergodic actions are conjugate if their spectral functors are related by a quasi-tensor natural unitary transformation. Explicitly, if $(\bar{L}, \widetilde{\bar{L}})$ and $\left(\bar{L}^{\prime}, \tilde{\bar{L}}^{\prime}\right)$ are the spectral functors of the actions, we need a unitary $U_{u}: \bar{L}_{u} \rightarrow \bar{L}_{u}^{\prime}$ for each $u \in \operatorname{Rep}\left(G_{\tau}\right)$, natural in $u$, such that $U_{u \otimes v} \circ \tilde{\bar{L}}_{u, v}=\tilde{\bar{L}}_{u, v}^{\prime} \circ U_{u} \otimes U_{v}$ and $U_{\iota}=1_{\iota}$. Now the spectral space of the ergodic action constructed from $(\tau, \mu)$ is $\bar{L}_{u}:=\left(\iota, \mu_{u}\right)$, and similarly for $\left(\tau, \mu^{\prime}\right)$. Note that $\phi$, as a map between the Hilbert spaces $\left(\iota, \mu_{u}\right)$ and $\left(\iota, \mu_{u}^{\prime}\right)$ is isometric and in fact unitary, since $\phi$ is a full functor. An easy computation shows that the collection of these unitaries satisfies the needed relations.

Our first result concerns the construction of induced bimodule representations. The construction reduces to $(\mathcal{\ell}, \alpha)$ for $u=\iota$.

Theorem 6.2. Pick an object $u$ of $\mathcal{A}$ with $1_{\mu_{u}} \neq 0$.

(a) The linear space ${ }^{\circ} \mathcal{H}_{u}$ obtained quotienting $\sum_{v}\left(\mu_{v}, \mu_{u}\right) \otimes \tau_{v}$ by the linear subspace generated by elements of the form $M \circ \mu(A) \otimes \psi-M \otimes \tau(A) \circ \psi$ can be naturally completed into a nonzero full Hilbert module $\mathscr{H}_{u}$ over $\mathcal{C}$, with a faithful left action of $\mathcal{C}$ making it into a Hilbert bimodule over $\mathcal{C}$. $\mathscr{H}_{u}$ depends only on $\mu_{u}$. 
(b) There is a unique, full, bimodule representation, $\operatorname{Ind}\left(\mu_{u}\right)$, of $G_{\tau}$ on $\mathscr{H}_{u}$ with

$$
\operatorname{Ind}\left(\mu_{u}\right) M \otimes \psi=M \otimes \hat{v} \psi
$$

for $M \in\left(\mu_{v}, \mu_{u}\right), \psi \in \tau_{v}, \hat{v}$ being the representation of $G_{\tau}$ on $\tau_{v}$.

Theorem 6.2 will be proved in Sections 7 and 8. In Section 8, we shall see that the map Ind: $\mu_{u} \rightarrow \operatorname{Ind}\left(\mu_{u}\right)$ extends to a ${ }^{*}$-functor Ind: $\mathcal{M}_{\mu} \rightarrow \operatorname{Bimod}_{\alpha}\left(G_{\tau}\right)$ on the full $\mathrm{C}^{*}$-subcategory $\mathcal{M}_{\mu}$ of $\mathcal{M}$ whose objects are those in the image of $\mu$. It will be called the induction functor.

In Section 8, we shall also prove the following analogue of Swan's theorem in our framework. The assumptions are those of Theorem 6.2. Ind $\mu$ denotes the composed functor.

Theorem 6.3. (a) For any object $u$ of $\mathcal{A}$ there is a natural isometric intertwiner of Hilbert module representations $S_{u} \in\left(\operatorname{Ind}\left(\mu_{u}\right), \hat{u} \otimes \alpha\right)$. In particular, if $\mu$ is relaxed tensor, then $S_{u}$ is unitary. Hence $\mathscr{H}_{u}$ is always finite projective as a right module and free if $\mu$ is relaxed tensor.

(b) For any arrow $A \in(u, v)$, Ind $\mu(A)$ corresponds to the restriction of $\tau(A) \otimes I$ to the space of the associated subrepresentation of $\hat{u} \otimes \alpha$ under $S_{u}$.

In other words, for any arrow $A \in\left(u, u^{\prime}\right)$ in $\mathcal{A}$, the following diagram commutes.

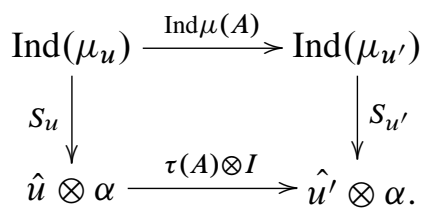

Note that if $\mu$ is just quasi-tensor, $\mathcal{M}_{\mu}$ may not be a tensor category. We ask, however, whether Ind extends tensorially to the smallest full tensor subcategory $\mathcal{M}_{\mu}^{\otimes}$ of $\mathcal{M}$ generated by $\mathcal{M}_{\mu}$. Somewhat surprisingly, the answer is that it does. Sections 9 and 10 will be devoted to discussing the following result, a generalization of Theorem 4.1 to a noncommutative framework.

Theorem 6.4. The induction functor Ind: $\mu_{u} \in \mathcal{M}_{\mu} \rightarrow \operatorname{Ind}\left(\mu_{u}\right) \in \operatorname{Bimod}_{\alpha}\left(G_{\tau}\right)$ extends uniquely to a full and faithful strict tensor functor to a strict tensor category of Hilbert bimodule representations

$$
\text { Ind : } \mathcal{M}_{\mu}^{\otimes} \rightarrow \operatorname{Bimod}_{\alpha}\left(G_{\tau}\right) \text {. }
$$

Furthermore, (Ind, $\mu$ ) gives rise to an adjoint pair of functors.

Since Ind is a strict tensor functor, the composed functor Ind $\mu: \mathcal{A} \rightarrow \operatorname{Bimod}_{\alpha}\left(G_{\tau}\right)$ is quasi-tensor, relaxed tensor or strict tensor according as $\mu$ is. The associated natural 
transformation Ind $\mu$ is computed in Section 9. Moreover, since Ind is full, we may regard it as an isomorphism between the original functor $(\mu, \tilde{\mu})$ and (Ind $\mu, \widetilde{\operatorname{Ind} \mu})$. In the following commutative diagram, the dotted arrows summarize our construction from the given pair $(\tau, \mu)$.

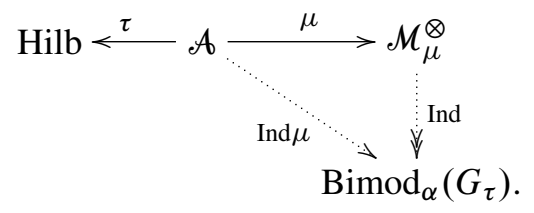

Remark. Combining Theorems 6.3 and 6.4 yields an explicit description of the quasitensor functor $\mu: \mathcal{A} \rightarrow \mathcal{M}_{\mu}^{\otimes}$ in terms of $\tau$ and the ergodic action $(\mathcal{C}, \alpha)$. This is then used for the embedding results, Theorem 6.7 and Corollaries 6.8 and 6.9.

We next give two applications of our results, that originally motivated our work. The first concerns a tensor $\mathrm{C}^{*}$-category with conjugates whose object set contains a distinguished generating element. We give two results, corresponding to the selfconjugate or non-selfconjugate case.

Theorem 6.5. Let $\mathcal{M}$ be a tensor $C^{*}$-category with objects $\iota, x, x^{2}, \ldots$, where $x$ is a real or pseudoreal object defined by a solution $R \in\left(\iota, x^{2}\right)$ of the conjugate equations with $\|R\|^{2} \geq 2$. Let $F \in \operatorname{Mat}_{n}(\mathbb{C})$ be an invertible matrix with $\operatorname{Tr}\left(F F^{*}\right)=\operatorname{Tr}\left(\left(F F^{*}\right)^{-1}\right)=\|R\|^{2}$. Then there is a full and faithful tensor functor $\mathcal{M} \rightarrow \operatorname{Bimod}_{\alpha}\left(A_{o}(F)\right)$, where $\alpha$ is the ergodic action of $A_{o}(F)$ on $C$ associated to $(\tau, \mu)$.

Theorem 6.6. If the set of objects of $\mathcal{M}$ is generated, as a semigroup, by $x$ and a conjugate $\bar{x}$, with intrinsic dimension $d(x) \geq 2$, then conclusions analogous to Theorem 6.5 hold where the quantum group is now $A_{u}(F)$.

Examples. Note that any spectral functor of an ergodic action of a compact quantum group arises from some pair $(\tau, \mu)$, as we may choose for $\tau: \operatorname{Rep}(G) \rightarrow$ Hilb the embedding functor and for $\mu$ the spectral functor of the action, $\mu:=\bar{L}: \operatorname{Rep}(G) \rightarrow$ Hilb. (Recall that $\bar{L}$ is quasi-tensor by [34], cf. Section 2.) On the other hand, many examples of noncommutative ergodic spaces are known to arise from pairs $(\tau, \mu)$, with $\tau$ as above, but where $\mu$ is tensorial or relaxed tensorial. For example, compact quantum quotients (completed in the maximal $\mathrm{C}^{*}$-norm) $C(K \backslash G)$ arise from the restriction functor $\mu: \operatorname{Rep}(G) \rightarrow \operatorname{Rep}(K)$ [34]. The examples with high multiplicities of [2] are associated with the composition $\mu$ of a tensorial isomorphism with the embedding functor, $\mu: \operatorname{Rep}(G) \simeq \operatorname{Rep}\left(G^{\prime}\right) \rightarrow$ Hilb. Examples of categories of the type described in Theorems 6.5 and 6.6 arise from inclusions of $\mathrm{II}_{1}$ factors $N \subset M$ with finite Jones index. The ergodic action corresponding to the real object ${ }_{N} M_{N}$ is made explicit in [36]. For any finite index inclusion of infinite factors described by an 
endomorphism $\rho$ with $d(\rho) \geq 2$, the tensor $\mathrm{C}^{*}$-category generated by $\rho$ and $\bar{\rho}$ is of the form described in Theorem 6.6.

The proofs of Theorems 6.5 and 6.6 will be given at the end of Section 10. The next application concerns tensor categories $\mathcal{M}$ extending the representation category of a compact group. The following results, discussed in Sections 11 and 12, shed light on the problem of recognizing which tensor categories can be embedded into Hilbert spaces. They are obtained combining our bimodule construction with the work of Takesaki [43], Høegh-Krohn, Landstad and Størmer [15] and Wassermann [46].

In the following theorem $G$ is a compact Lie group, and we fix a distinguished faithful representation $u$ such that every irreducible of $G$ is a subrepresentation of a tensor power of $u$. We denote by $\delta_{G}$ be the full subcategory of $\operatorname{Rep}(G)$ with objects $\iota, u, u^{2}, \ldots$

Theorem 6.7. Let $G$ be a compact Lie group with a distinguished faithful representation $u$, and let $\mu: \delta_{G} \rightarrow \mathcal{M}$ be a tensor functor. Let $\mathcal{C}$ be the ergodic $C^{*}$-algebra associated with $\mu$ and the embedding functor $\tau$ of $S_{G}$ into the category of Hilbert spaces. Assume that the von Neumann algebra $\mathcal{C}^{\prime \prime}$ generated by $\mathcal{C}$ in the GNS representation of the unique $G$-invariant trace is of type I and let $K$ be a closed subgroup of $G$ such that $L^{\infty}(K \backslash G) \simeq Z\left(\mathcal{C}^{\prime \prime}\right)$ as ergodic $W^{*}$-systems. Then there is a full and faithful tensor functor $\epsilon: \mathcal{M}_{\mu}^{\otimes} \rightarrow \operatorname{Rep}(K)$.

Notice that in the above theorem $\mathcal{M}_{\mu}^{\otimes}$ is simply the full subcategory of $\mathcal{M}$ with objects the tensor powers of $\mu_{u}$.

Remark. As we shall see in Section 11, the functor $\epsilon$ is naturally associated with $\mu$. However, the set of objects in the image of $\epsilon$ in general does not generate all the representations of $K$ under tensor products, subobjects and direct sums. In fact, in the particular case where each irreducible of $G$ has multiplicity in $\mathcal{C}$ equal to its dimension, then $\epsilon$ maps each object to the trivial representation of $K$. Hence $\mathcal{M}_{\mu}^{\otimes}$ admits a tensor functor to a full subcategory of the category of Hilbert spaces. Furthermore, any full multiplicity ergodic action of $G$ on a type I von Neumann algebra arises from a relaxed tensor functor $\mu$, its spectral functor.

At the other extreme, if $\mathcal{C}$ is commutative, as happens in particular if $\mathcal{M}$ has permutation symmetry, see [34], then we get the following result, generalizing an important step in [11] towards proving the abstract duality theorem for compact groups.

Corollary 6.8. If $\mathcal{C}$ is commutative, and hence $\mathcal{C}=C(K \backslash G)$ as ergodic $C^{*}$-systems, then $\epsilon\left(\mu_{u}\right)=u \uparrow_{K}$. Hence the completion of the image of $\epsilon$ under subobjects contains any irreducible of $K$. 
It is still an open problem whether ergodic actions of classical compact Lie groups $G$ on von Neumann algebras are always of type I. By Theorem 6.7, a positive answer for a specific group $G$ would guarantee the existence of an embedding for all tensor $\mathrm{C}^{*}$-categories $\mathcal{M}$ containing the representation category of $G$ and having the same objects. Wassermann has shown this to be true for $G=\mathrm{SU}(2)$ [46]. Taking the known abstract characterization of $\operatorname{Rep}(\mathrm{SU}(2))$ into account [10], see also [32], [34], we obtain the following embedding result for tensor $\mathrm{C}^{*}$-categories containing a distinguished pseudoreal object of dimension 2. No permutation symmetry is assumed.

Corollary 6.9. Let $\mathcal{M}$ be a tensor $C^{*}$-category whose object semigroup is generated by a pseudoreal object $x$ of dimension 2, i.e., with an intertwiner $R \in\left(\iota, x^{2}\right)$ such that

$$
R^{*} \otimes 1_{x} \circ 1_{x} \otimes R=-1_{x}, \quad\|R\|^{2}=2 .
$$

Then there is a closed subgroup $K$ of $\mathrm{SU}(2)$ and a full and faithful tensor functor $\mathcal{M} \rightarrow \operatorname{Rep}(K)$.

Remarks and more results. A non-trivial problem is to construct new examples of or even classify the quasi-tensor functors $\mu: \mathcal{A} \rightarrow \mathcal{M}$, for a given embeddable tensor $C^{*}$-category $\mathcal{A}$. Our results connect this problem to that of classifying the ergodic $\mathrm{C}^{*}$-actions of the quantum group $G_{\tau}$ associated to an embedding $\tau$ of $\mathcal{A}$. On one hand, as recalled in the examples previously discussed, every spectral functor of an ergodic action of $G_{\tau}$ on a unital $C^{*}$-algebra arises in this way. Even for ergodic $\mathrm{C}^{*}$-actions of compact groups, where there are important results, not a lot is known (cf. Section 2). In the quantum case very little is known, but it is already clear that there are many new aspects.

Motivated by our applications, we are especially interested in the case where $\mu$ is tensorial or relaxed tensorial. The reconstruction results, Theorems 6.2 and 6.4 , then lead to the problem of classifying those ergodic actions $(\ell, \alpha)$ where the module representations $u \otimes \alpha$ (or a subrepresentation on a projective module in the quasi-tensor case) can be made into full bimodule representations.

Not all ergodic actions, even of compact groups can arise in this way. In Section 11 we classify full bimodule representations arising from ergodic actions of compact groups on type I von Neumann algebras. This provides an obstruction to the existence of full bimodule representations (and hence to the existence of relaxed tensor functors $\mu: \mathcal{A} \rightarrow \mathcal{M}$ ) in the case of low but nonzero multiplicities. For example, we derive that neither the ergodic actions with full spectrum and irreducibles of low multiplicity nor the adjoint action by a non-trivial irreducible representation of SU(2) can arise.

\section{Algebraic bimodules from pairs of functors}

As in the previous section, we start with tensor $\mathrm{C}^{*}$-categories $\mathcal{A}$ and $\mathcal{M}$ and we assume that $\mathcal{A}$ has conjugates. Let $(\mu, \tilde{\mu}): \mathcal{A} \rightarrow \mathcal{M}$ be a quasi-tensor functor 
and $\tau: \mathcal{A} \rightarrow$ Hilb a tensor functor into the category of Hilbert spaces. We have an associated unital $C^{*}$-algebra $\mathcal{C}$, the completion of a canonical dense ${ }^{*}$-subalgebra ${ }^{\circ} \mathcal{C}$. In this section we generalize that construction at the algebraic level to get bimodules over ${ }^{\circ} \mathrm{C}$. The norm completion and the quantum group action will be considered in the next section.

7.1. The algebraic bimodules ${ }^{\circ} \mathscr{H}_{u}$. Pick an object $u$ of $\mathcal{A}$. Let ${ }^{\circ} \mathscr{H}_{u}$ be the linear space $\sum_{v}\left(\mu_{v}, \mu_{u}\right) \otimes \tau_{v}$, the sum being taken over the objects of $\mathcal{A}$, quotiented by the linear subspace generated by elements of the form

$$
M \circ \mu(A) \otimes \psi-M \otimes \tau(A) \circ \psi .
$$

Notice that, as the objects involved have conjugates [34], [35], and tensor units are irreducible, we are actually taking a sum of finite-dimensional vector spaces [22]. It should be noted that the bimodule ${ }^{\circ} \mathcal{H}_{u}$ in fact depends only on $\mu_{u}$.

We next introduce a multiplication and adjoint,

$$
\cdot:{ }^{\circ} \mathcal{H}_{u} \times{ }^{\circ} \mathcal{H}_{u^{\prime}} \rightarrow{ }^{\circ} \mathcal{H}_{u \otimes u^{\prime}}, \quad{ }^{*}:{ }^{\circ} \mathcal{H}_{u} \rightarrow{ }^{\circ} \mathcal{H}_{\bar{u}},
$$

to get a structure analogous to ${ }^{*}$-algebra. These operations will be used in Section 8 to simplify computations, the multiplication - also plays a role in Section 9.

7.2. The multiplication $\xi \cdot \eta$. For simple tensors $\xi=L \otimes \psi \in{ }^{\circ} \mathcal{H}_{u}, \eta=M \otimes \phi \in$ ${ }^{\circ} \mathcal{H}_{u^{\prime}}$, with $L \in\left(\mu_{w}, \mu_{u}\right), M \in\left(\mu_{v}, \mu_{u^{\prime}}\right), \psi \in \tau_{w}, \phi \in \tau_{v}$, set

$$
\xi \cdot \eta:=\tilde{\mu}_{u, u^{\prime}} \circ(L \otimes M) \circ \tilde{\mu}_{w, v}^{*} \otimes(\psi \otimes \phi) .
$$

It is easy to check that these maps are well defined and associativity follows from that of the functors $\mu$ and $\tau$. In particular, ${ }^{\circ} \mathscr{H}_{l}$ is an algebra, denoted above by ${ }^{\circ} \mathcal{C}$. Note that the multiplication depends in general on $u$ and $u^{\prime}$ and not only on $\mu_{u}$, $\mu_{u^{\prime}}$. However, if $u^{\prime}$ (or $u$ ) is the tensor unit, it depends only on $\mu_{u}$ (or $\mu_{u^{\prime}}$ ) as $\tilde{\mu}_{u, \iota}=\tilde{\mu}_{\iota, u}=1_{\mu_{u}}$. Hence

$$
{ }^{\circ} \mathcal{H}_{u} \text { is a }{ }^{\circ} \mathcal{C} \text {-bimodule depending just on } \mu_{u} \text {. }
$$

7.3. The functor $\lambda$. We define a functor, denoted by $\lambda$ for the moment, from $\mathcal{M}_{\mu}$ to the category of ${ }^{\circ} \mathcal{C}$-bimodules. After the norm and the quantum group action have been introduced, $\lambda$ will be the induction functor Ind.

Given $Y \in\left(\mu_{u}, \mu_{u^{\prime}}\right)$, we define a map

$$
\lambda(Y):{ }^{\circ} \mathcal{H}_{u} \rightarrow{ }^{\circ} \mathcal{H}_{u^{\prime}}, \quad \lambda(Y)(M \otimes \psi):=(Y \circ M) \otimes \psi .
$$

It is easily checked that $\lambda(Y)$ is a bimodule map so $\lambda$ is a covariant functor, from the full subcategory $\mathcal{M}_{\mu}$ of $\mathcal{M}$ whose objects are the images of objects of $\mathcal{A}$ into the category of ${ }^{\circ} \mathcal{C}$-bimodules. 
7.4. The adjoint $\xi^{*}$. We next define an adjoint on these bimodules. Here matters are slightly more complicated.

As recalled in Section 2.2, if $(\mu, \tilde{\mu})$ is a quasi-tensor functor and if $R, \bar{R}$ defines a conjugate for an object $u$, then $\widehat{R}:=\tilde{\mu}_{\bar{u}, u}^{*} \circ \mu(R)$ and $\widehat{\bar{R}}:=\tilde{\mu}_{u, \bar{u}}^{*} \circ \mu(\bar{R})$ is a solution of the conjugate equations for $\mu_{u}$, the image solution of $R, \bar{R}$ under $\mu$ [34].

Fixing an object $u \in \mathcal{A}$ and a solution $R, \bar{R}$ of the conjugate equations for $u$, we associate an antilinear map ${ }^{*}: \mathscr{H}_{u} \rightarrow \mathscr{H}_{\bar{u}}$ in the following way. Choose solutions of the conjugate equations $v \rightarrow R_{v}, \bar{R}_{v}$ of for all objects of $\mathcal{A}$. Set

$$
(M \otimes \psi)^{*}:=M^{\bullet} \otimes j_{v} \psi
$$

for $M \in\left(\mu_{v}, \mu_{u}\right), \psi \in \tau_{v}$, where ${ }^{\bullet}:\left(\mu_{v}, \mu_{u}\right) \rightarrow\left(\mu_{\bar{v}}, \mu_{\bar{u}}\right)$ is defined using image solutions under $\mu$ of the chosen solutions $R_{v}, \bar{R}_{v}$ for the running objects $v$ appearing in the sum and $R, \bar{R}$ for the fixed object $u$ respectively and $j_{v}:=j_{\tau_{v}}$ corresponds to $\tau\left(R_{v}\right)$ and $\tau\left(\bar{R}_{v}\right)$ as in Section 2.1. Notice that ${ }^{*}$ is well defined by the compatibility properties with $\circ, \mu$ and $\tau$, see Sections 2.1 and 2.2. Moreover, ${ }^{*}$ is independent of the choice $v \rightarrow R_{v}, \bar{R}_{v}$ in $\mathcal{A}$ for the running objects $v$, as if $Y \in(\bar{v}, \tilde{v})$ is an invertible, $M^{\bullet}$ and $j_{v} \psi$ become $M^{\bullet} \circ \mu\left(Y^{-1}\right)$ and $\tau(Y) j_{v} \circ \psi$ respectively. However, if we change the solution of the conjugate equations for $u$ using an $X \in(\bar{u}, \tilde{u}),(M \otimes \psi)^{*}$ becomes $\left(\mu(X) M^{\bullet}\right) \otimes\left(j_{v} \psi\right)=\lambda(\mu(X))(M \otimes \psi)^{*}$, hence the associated ${ }^{*}$ changes. This unpleasant feature will play no role in the construction of the bimodule representation.

We note that for $u=\iota$ the ${ }^{*}$-operation is independent and makes ${ }^{\circ} \mathcal{C}$ into a unital *-algebra.

\subsection{Compatibility of the various operations}

Proposition 7.1. Let $u$, $u^{\prime}$ be objects of $\mathcal{A}$. If $\xi \in{ }^{\circ} \mathscr{H}_{u}$ and $\xi^{\prime} \in{ }^{\circ} \mathscr{H}_{u^{\prime}}$, then $\left(\xi \cdot \xi^{\prime}\right)^{*}=\xi^{\prime *} \cdot \xi^{*}$ and $\xi^{* *}=\xi$, where we have used tensor product solutions of the conjugate equations for $u \otimes u^{\prime}$ and conjugate solutions for $\bar{u}$.

Proof. Write $\xi:=L \otimes \psi$ and $\xi^{\prime}:=M \otimes \phi$ with $L \in\left(\mu_{w}, \mu_{u}\right), \psi \in \tau_{w}, M \in$ $\left(\mu_{v}, \mu_{u^{\prime}}\right), \phi \in \tau_{v}$. We may compute $\left(\xi \cdot \xi^{\prime}\right)^{*}$ using image of a tensor product solution of the conjugate equations for $w$ and $v$. The first result follows from

$$
\left(\mu_{u, u^{\prime}} \circ L \otimes M \circ \tilde{\mu}_{w, v}^{*}\right)^{\bullet}=\mu_{\bar{u}^{\prime}, \bar{u}} \circ M^{\bullet} \otimes L^{\bullet} \circ \tilde{\mu}_{\bar{v}, \bar{w}}^{*} ;
$$

see Section 2.1 for the compatibility properties of $\bullet$ with $\otimes$ and $\circ$ and Corollary 13.3 for the explicit computations of $\mu_{w, v}^{*} \bullet$ and $\mu_{u, u^{\prime}}^{\bullet}$. The second result follows from $M^{\bullet \bullet}=M$ when a solution of the conjugate equation and successively the conjugate solution is used.

Remark. Some care is required in using this proposition. For example, if $u$ is pseudoreal and irreducible and we use $R \in\left(\iota, u^{2}\right)$ to define $\xi^{*}$ in $\mathscr{H}_{u}$, we must use $-R$ to define $\xi^{* *}$ in $\mathscr{H}_{u}$. 
Proposition 7.2. For $Y \in\left(\mu_{u}, \mu_{u^{\prime}}\right), \xi \in{ }^{\circ} \mathscr{H}_{u}, \xi^{\prime} \in{ }^{\circ} \mathcal{H}_{u^{\prime}}, A \in(u, z), A^{\prime} \in\left(u^{\prime}, z^{\prime}\right)$,

$$
\begin{aligned}
(\lambda(Y) \xi)^{*} & =\lambda\left(Y^{\bullet}\right) \xi^{*}, \\
\lambda\left(\mu\left(A \otimes A^{\prime}\right)\right) \xi \cdot \xi^{\prime} & =\lambda(\mu(A)) \xi \cdot \lambda\left(\mu\left(A^{\prime}\right)\right) \xi^{\prime} .
\end{aligned}
$$

The last item to be introduced in this section is a sesquilinear form on the bimodules ${ }^{\circ} \mathcal{H}_{u}$. It will be shown to be positive in the next section, allowing us to pass from the algebraic to the analytic level.

7.6. The sesquilinear form on the bimodules ${ }^{\circ} \mathscr{H}_{\boldsymbol{u}}$. We retain the notation of subsect. 7.4 and define ${ }^{\circ} \mathcal{C}$-valued form on ${ }^{\circ} \mathcal{H}_{u}$ by setting

$$
\left\langle\xi, \xi^{\prime}\right\rangle:=\lambda\left(\mu(R)^{*}\right)\left(\xi^{*} \cdot \xi^{\prime}\right) .
$$

The explicit formula, for $\xi=M \otimes \psi, \xi^{\prime}=M^{\prime} \otimes \psi^{\prime}$, with $M \in\left(\mu_{v}, \mu_{u}\right), \psi \in \tau_{v}$, $M^{\prime} \in\left(\mu_{v^{\prime}}, \mu_{u}\right), \psi^{\prime} \in \tau_{v^{\prime}}$, is

$$
\left\langle\xi, \xi^{\prime}\right\rangle:=\left(\hat{R}^{*} \circ M^{\bullet} \otimes M^{\prime} \circ \tilde{\mu}_{\bar{v}, v^{\prime}}^{*}\right) \otimes\left(j_{v} \psi \otimes \psi^{\prime}\right) .
$$

Remark. This form does not depend on the chosen solution of the conjugate equations for $u$. Indeed, if we change solution using an invertible $X$, then $M^{\bullet}$ becomes $\mu(X) \circ$ $M^{\bullet}$. This cancels the simultaneous change of $\widehat{R}^{*}$, which becomes $\widehat{R}^{*} \circ \mu\left(X^{-1}\right) \otimes 1_{\mu_{u}}$.

We conclude this section with an explicit computation of the right-hand side needed later.

Lemma 7.3. For $\xi=M \otimes \psi, \xi^{\prime}=M^{\prime} \otimes \psi^{\prime}, M \in\left(\mu_{v}, \mu_{u}\right), M^{\prime} \in\left(\mu_{v^{\prime}}, \mu_{u}\right)$, $\psi \in \tau_{v}, \psi^{\prime} \in \tau_{v^{\prime}}$

$$
\left\langle\xi, \xi^{\prime}\right\rangle=\left(\hat{R}_{v}^{*} \circ 1_{\mu_{\bar{v}}} \otimes\left(M^{*} \circ M^{\prime}\right) \circ \tilde{\mu}_{\bar{v}, v^{\prime}}^{*}\right) \otimes\left(j_{v} \psi \otimes \psi^{\prime}\right) .
$$

In particular, the form depends only on $\mu_{u}$.

Proof. We have

$$
\begin{aligned}
\hat{R}^{*} \circ & M^{\bullet} \otimes M^{\prime} \\
& =\hat{R}^{*} \circ\left[\left(\hat{R}_{v}^{*} \otimes 1_{\mu_{\bar{u}}} \circ 1_{\mu_{\bar{v}}} \otimes M^{*} \otimes 1_{\mu_{\bar{u}}} \circ 1_{\mu_{\bar{v}}} \otimes \hat{\bar{R}}\right) \otimes M^{\prime}\right] \\
& =\hat{R}_{v}{ }^{*} \otimes \hat{R}^{*} \circ 1_{\mu_{\bar{v}}} \otimes M^{*} \otimes 1_{\mu_{\bar{u}}} \otimes 1_{\mu_{u}} \circ 1_{\mu_{\bar{v}}} \otimes \hat{\bar{R}} \otimes 1_{\mu_{u}} \circ 1_{\mu_{\bar{v}}} \otimes M^{\prime} \\
& =\hat{R}_{v}{ }^{*} \circ 1_{\mu_{\bar{v}}} \otimes\left(M^{*} \circ M^{\prime}\right) .
\end{aligned}
$$

We have already noted that the form does not depend on the solution of the conjugate equations for $v$ and $u$ and see now that it does not change if we replace $u$ by another object $u^{\prime}$ such that $\mu_{u}=\mu_{u^{\prime}}$. 


\section{The induced Hilbert bimodule representations}

In this section we consider both the analytic aspect of the bimodules ${ }^{\circ} \mathcal{H}_{u}$ and the quantum group action, leading to the proof of Theorems 6.2 and 6.3.

Recall that $G_{\tau}$ was defined in Section 6 as the compact quantum group defined by the functor $\tau$ via Woronowicz duality. Let $\alpha$ be the action of $G_{\tau}$ on ${ }^{\circ} \mathcal{C}$ defined by $\alpha(M \otimes \psi)=M \otimes \hat{v}(\psi)$ for $M \in\left(\mu_{v}, \iota\right), \psi \in \tau_{v}$, where $\hat{v}$ denotes the representation of $G_{\tau}$ on $\tau_{v} .{ }^{\circ} \mathcal{C}$ is known to have a maximal $\mathrm{C}^{*}$-norm and $\alpha$ to extend uniquely to an ergodic action of $G_{\tau}$ on the completed C*-algebra $C^{*}$ [34].

8.1. Positivity of the sesquilinear form. Given objects $u, v \in \mathcal{A}, \phi \in \tau_{v}$, we let

$$
L_{u}(\phi):{ }^{\circ} \mathscr{H}_{u} \rightarrow{ }^{\circ} \mathscr{H}_{v} \otimes u
$$

be the operator of left multiplication by $1_{\mu_{v}} \otimes \phi \in{ }^{\circ} \mathcal{H}_{v}$ on ${ }^{\circ} \mathscr{H}_{u}$, hence obviously a right module morphism. Now set

$$
L_{u}(\phi)^{*}:{ }^{\circ} \mathcal{H}_{v \otimes u} \rightarrow{ }^{\circ} \mathcal{H}_{u}, \quad L_{u}(\phi)^{*}:=\lambda\left(\mu\left(R_{v}^{*} \otimes 1_{u}\right)\right) L_{v \otimes u}\left(j_{v} \phi\right) .
$$

If we change solutions of the conjugate equations using an invertible $X \in(\bar{v}, \tilde{v})$, it is not difficult to verify that, by Lemma 8.2 (c) below, $L_{u}(\phi)^{*}$ does not change.

Lemma 8.1. $\left\langle\eta, L_{u}(\phi)^{*} \xi\right\rangle=\left\langle L_{u}(\phi) \eta, \xi\right\rangle$.

Proof. We have

$$
\begin{aligned}
\left\langle\eta, L_{u}(\phi)^{*} \xi\right\rangle & =\lambda\left(\mu\left(R_{u}^{*}\right)\right)\left(\eta^{*} \cdot\left(\lambda\left(\mu\left(R_{v}^{*} \otimes 1_{u}\right)\right)\left(1_{\mu_{v}} \otimes \phi\right)^{*} \cdot \xi\right)\right) \\
& =\lambda\left(\mu\left(R_{u}^{*} \circ 1_{\bar{u}} \otimes R_{v}^{*} \otimes 1_{u}\right)\right) \eta^{*} \cdot\left(1_{\mu_{v}} \otimes \phi\right)^{*} \cdot \xi \\
& =\left\langle L_{u}(\phi) \eta, \xi\right\rangle .
\end{aligned}
$$

The second equality follows from Proposition 7.2 while in the last we have chosen product solutions of the conjugate equations for $v \otimes u$.

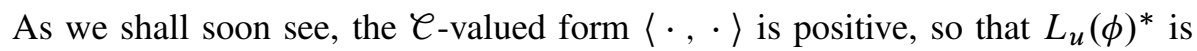
the adjoint of $L_{u}(\phi)$ as the notation suggests. These maps satisfy the following properties.

Lemma 8.2. (a) $\lambda\left(\tilde{\mu}_{r, w} \circ 1_{\mu_{r}} \otimes Y \circ \tilde{\mu}_{r, u}^{*}\right) L_{u}(\phi)=L_{w}(\phi) \lambda(Y), Y \in\left(\mu_{u}, \mu_{w}\right)$,

(b) $L_{u}(\phi)^{*} L_{u}(\psi)=\langle\phi, \psi\rangle$,

(c) $\lambda \mu\left(A \otimes 1_{u}\right) L_{u}(\phi)=L_{u}(\tau(A) \phi)$,

(d) $\sum_{i} L_{u}\left(\phi_{i}\right) L_{u}\left(\phi_{i}\right)^{*}=\lambda\left(\tilde{\mu}_{z, u} \circ \tilde{\mu}_{z, u}^{*}\right)$, where $\phi_{i}$ is an orthonormal basis of $\tau_{z}$. 
For $u=\iota$ the corresponding maps ${ }^{\circ} \mathcal{C} \rightarrow{ }^{\circ} \mathcal{H}_{z},{ }^{\circ} \mathcal{H}_{z} \rightarrow{ }^{\circ} \mathcal{C}$ will simply be denoted by $L(\phi)$ and $L(\phi)^{*}$. By (d), we have

$$
\sum L\left(\phi_{i}\right) L\left(\phi_{i}\right)^{*}=1
$$

for any orthonormal basis $\left(\phi_{i}\right)$ of $\tau_{z}$. We shall use this relation to define a faithful right module map $S_{z}:{ }^{\circ} \mathscr{H}_{z} \rightarrow \tau_{z} \otimes{ }^{\circ} \mathcal{C}$ by

$$
S_{z} \xi:=\sum_{i} \phi_{i} \otimes L\left(\phi_{i}\right)^{*} \xi,
$$

clearly independent of the choice of the orthonormal basis.

We are now ready to show positivity of the sesquilinear form of ${ }^{\circ} \mathcal{H}_{u}$.

Proposition 8.3. If $\tau_{z} \otimes^{\circ} \mathcal{C}$ is considered as a right prehilbertian ${ }^{\circ} \mathcal{C}$-module, the map $S_{z}$ satisfies

$$
\left\langle S_{z} \xi, S_{z} \xi^{\prime}\right\rangle=\left\langle\xi, \xi^{\prime}\right\rangle, \quad \xi, \xi^{\prime} \in{ }^{\circ} \mathscr{H}_{z}
$$

Hence ${ }^{\circ} \mathscr{H}_{z}$ is a finite projective, right prehilbertian module over ${ }^{\circ} \mathcal{C}$ with the sesquilin-

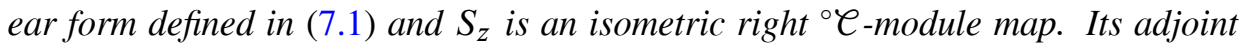
$S_{z}^{*}: \tau_{z} \otimes{ }^{\circ} \mathcal{C} \rightarrow{ }^{\circ} \mathscr{H}_{z}$ is given by

$$
S_{z}^{*}(\psi \otimes I)=1_{\mu_{z}} \otimes \psi
$$

for $\psi \in \tau_{z}$.

Proof. We have

$$
\left\langle S_{z} \xi, S_{z} \xi^{\prime}\right\rangle=\sum_{i}\left\langle L\left(\phi_{i}\right)^{*} \xi, L\left(\phi_{i}\right)^{*} \xi^{\prime}\right\rangle=\sum_{i}\left\langle\xi, L\left(\phi_{i}\right) L\left(\phi_{i}\right)^{*} \xi^{\prime}\right\rangle=\left\langle\xi, \xi^{\prime}\right\rangle .
$$

Hence $\langle\cdot, \cdot\rangle$ is a faithful, positive, ${ }^{\circ} \mathcal{C}$-valued inner product on ${ }^{\circ} \mathscr{H}_{z}$ and $S_{z}$ an isometry. We next compute the adjoint of $S_{z}$. If $\xi \in{ }^{\circ} \mathscr{H}_{z}, \psi \in \tau_{z}$, then

$$
\begin{aligned}
\left\langle\xi, S_{z}^{*} \psi \otimes I\right\rangle & =\left\langle S_{z} \xi, \psi \otimes I\right\rangle \\
& =\sum_{i}\left\langle\phi_{i} \otimes L\left(\phi_{i}\right)^{*} \xi, \psi \otimes I\right\rangle \\
& =\sum_{i}\left(\phi_{i}, \psi\right)\left\langle\xi, 1_{z} \otimes \phi_{i}\right\rangle=\left\langle\xi, 1_{z} \otimes \psi\right\rangle,
\end{aligned}
$$

as required.

We next compute the range projection $P_{z}=S_{z} S_{z}^{*}$ to see when $S_{z}$ is unitary. If $\psi \in \tau_{z}$, then

$$
\begin{aligned}
P_{z}(\psi \otimes I) & =S_{z}\left(1_{\mu_{z}} \otimes \psi\right) \\
& =\sum_{i} \phi_{i} \otimes L\left(\phi_{i}\right)^{*}\left(1_{\mu_{z}} \otimes \psi\right) \\
& =\sum_{i} \phi_{i} \otimes\left(\left(\mu\left(R_{z}^{*}\right) \circ \tilde{\mu}_{\bar{z}, z} \circ \tilde{\mu}_{\bar{z}, z}^{*}\right) \otimes\left(j_{z} \phi_{i} \otimes \psi\right)\right) .
\end{aligned}
$$


Corollary 8.4. If $\tilde{\mu}_{\bar{z}, z} \circ \tilde{\mu}_{\bar{z}, z}^{*} \circ \mu\left(R_{z}\right)=\mu\left(R_{z}\right)$ (e.g., when $\mu$ is relaxed tensor), then $S_{z}$ is unitary, and hence ${ }^{\circ} \mathscr{H}_{z}$ is a free right ${ }^{\circ} \mathcal{C}$-module.

Proof. We now have

$$
\begin{aligned}
\mu\left(R_{z}^{*}\right) \circ \tilde{\mu}_{\bar{z}, z} \circ \tilde{\mu}_{\bar{z}, z}^{*} \otimes\left(j_{z} \phi_{i} \otimes \psi\right) & =\mu\left(R_{z}^{*}\right) \otimes\left(j_{z} \phi_{i} \otimes \psi\right) \\
& =1_{\iota} \otimes \tau\left(R_{z}^{*}\right) j_{z} \phi_{i} \otimes \psi=\left\langle\phi_{i}, \psi\right\rangle .
\end{aligned}
$$

Hence $P_{z}$ is the identity projection.

We conclude the subsection noting that property (c) of Lemma 8.2 implies that $S$ is a natural transformation from $\lambda \mu$ to $\tau \otimes I$, i.e., when $A \in\left(u, u^{\prime}\right)$, the following diagram commutes:

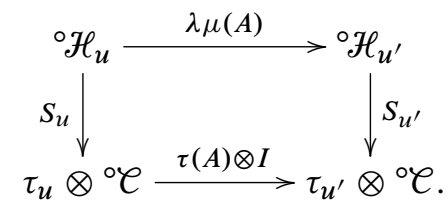

This will be used at the end of the section when proving Theorem 6.3, the generalized form of Swan's theorem.

\section{2. ${ }^{\circ} \mathscr{H}_{u}$ is algebraically full}

Proposition 8.5. Let $u$ be an object of $\mathcal{A}$ with $1_{\mu_{u}} \neq 0$. Then the coefficients $\left\langle\xi, \xi^{\prime}\right\rangle$, $\xi, \xi^{\prime} \in \mathscr{H}_{u}, \operatorname{span}^{\circ} \mathcal{C}$.

Proof. Choose $v=u, v^{\prime}=u \otimes v^{\prime \prime}, M=1_{\mu_{u}}, M^{\prime}=M^{\prime \prime} \circ \tilde{\mu}_{u, v^{\prime \prime}}^{*}$ with $M^{\prime \prime} \in$ $\left(\mu_{u} \otimes \mu_{v^{\prime \prime}}, \mu_{u}\right), \psi=j_{u}^{-1} \phi_{i}, \psi^{\prime}=j_{u}^{-1} \phi_{i} \otimes \psi^{\prime \prime}$ in (7.1), where $\left(\phi_{i}\right)$ is an orthonormal basis of $\tau_{\bar{u}}$ and $\psi^{\prime \prime} \in \tau_{v^{\prime \prime}}$. Summing over $i$ gives

$$
\begin{aligned}
\left(\hat{R}_{u}^{*} \circ 1_{\mu_{\bar{u}}} \otimes\left(M^{\prime \prime} \circ \tilde{\mu}_{u, v^{\prime \prime}}^{*}\right) \circ \tilde{\mu}_{\bar{u}, u \otimes v^{\prime \prime}}^{*}\right) \otimes\left(\tau\left(R_{u}\right) \otimes \psi^{\prime \prime}\right) \\
\quad=\left(\hat{R}_{u}^{*} \circ 1_{\mu_{\bar{u}}} \otimes\left(M^{\prime \prime} \circ \tilde{\mu}_{u, v^{\prime \prime}}^{*}\right) \circ \tilde{\mu}_{\bar{u}, u \otimes v^{\prime \prime}}^{*} \circ \mu\left(R_{u} \otimes 1_{v^{\prime \prime}}\right)\right) \otimes \psi^{\prime \prime} \\
\quad=\left(\hat{R}_{u}^{*} \circ 1_{\mu_{\bar{u}}} \otimes\left(M^{\prime \prime} \circ \tilde{\mu}_{u, v^{\prime \prime}}^{*}\right) \circ \tilde{\mu}_{\bar{u}, u \otimes v^{\prime \prime}}^{*} \circ \tilde{\mu}_{u} \otimes \bar{u}, v^{\prime \prime} \circ \mu\left(R_{u}\right) \otimes 1_{\mu_{v^{\prime \prime}}}\right) \otimes \psi^{\prime \prime} \\
\quad=\left(\hat{R}_{u}^{*} \circ 1_{\mu_{\bar{u}}} \otimes M^{\prime \prime} \circ \hat{R}_{u} \otimes 1_{\mu_{v^{\prime \prime}}}\right) \otimes \psi^{\prime \prime} .
\end{aligned}
$$

Now recall, see e.g. [22], that if $\rho, \sigma, \tau$ are objects of a tensor $\mathrm{C}^{*}$-category with conjugates, the map

$$
T \in(\rho \otimes \sigma, \tau) \rightarrow 1_{\bar{\rho}} \otimes T \circ R_{\rho} \otimes 1_{\sigma} \in(\sigma, \bar{\rho} \otimes \tau)
$$

is a linear isomorphism. Hence $X:=1_{\mu_{\bar{u}}} \otimes M^{\prime \prime} \circ \widehat{R}_{u} \otimes 1_{\mu_{v^{\prime \prime}}}$ is a generic element of $\left(\mu_{v^{\prime \prime}}, \mu_{\bar{u}} \otimes \mu_{u}\right)$ and can, in particular, be any element of the form $X=\widehat{R}_{u} \circ Y$ where $Y \in\left(\mu_{v^{\prime \prime}}, \iota\right)$. Hence the linear span of the coefficients of the inner product on ${ }^{\circ} \mathcal{H}_{u}$ is ${ }^{\circ} \mathcal{C}$ as it contains any element of the form $Y \otimes \psi^{\prime \prime}$. 
8.3. A useful formula for the left ${ }^{\circ} \mathcal{C}$-action on ${ }^{\circ} \mathscr{H}_{u}$. As $x_{i}:=S_{u}^{*}\left(\psi_{i} \otimes I\right)=$ $1_{\mu_{u}} \otimes \psi_{i}$, where $\left(\psi_{i}\right)$ is an orthonormal basis of $\tau_{u}$, is a Hilbert module basis, we need only specify $\left\langle x_{i}, c \cdot x_{j}\right\rangle$ for $c \in{ }^{\circ} \mathcal{C}$.

Proposition 8.6. If $c=T \otimes \phi \in{ }^{\circ} \mathcal{C}$, with $T \in\left(\mu_{v}, \iota\right), \phi \in \tau_{v}$, then

$$
\left\langle x_{i}, c \cdot x_{j}\right\rangle=\left(\hat{R}_{u}^{*} \circ 1_{\mu_{\bar{u}}} \otimes T \otimes 1_{\mu_{u}} \circ \tilde{\mu}_{\bar{u}, v, u}^{*}\right) \otimes\left(j_{u} \psi_{i} \otimes \phi \otimes \psi_{j}\right) .
$$

Example. Let $K \subset G$ be an inclusion of a compact quantum groups. Then we have a tensor functor $\mu: \operatorname{Rep}(G) \rightarrow \operatorname{Rep}(K)$ taking a representation $u$ of $G$ to its restriction $u \uparrow_{K}$ to the subgroup; see, e.g., [33]. Hence ${ }^{\circ} \mathscr{H}_{u}$ is a free module. ${ }^{\circ} \mathcal{C}$ is the canonical dense *-subalgebra of $C(K \backslash G)$. The above formula then gives:

$$
\left\langle x_{i}, c \cdot x_{j}\right\rangle=\sum_{r} u_{r i}^{*} c u_{r j}, \quad c \in{ }^{\circ} C(K \backslash G),
$$

see [34]. This example was discussed at the end of Section 5.

Example. Let $G$ be a compact quantum group acting ergodically on a unital $\mathrm{C}^{*}$ algebra $\mathcal{C}$, and let $\bar{L}: \operatorname{Rep}(G) \rightarrow$ Hilb be the spectral functor of the action as in [34], and shown there to be a quasi-tensor functor. Then ${ }^{\circ} \mathcal{C}$ is the ${ }^{*}$-algebra spanned by the elements of $\mathcal{C}$ transforming under the action like unitary irreducible $G$-representations. ${ }^{\circ} \mathcal{H}_{u} \neq 0$ precisely when $\bar{L}_{u} \neq 0$ and, if $u$ is irreducible, this is equivalent to requiring $u$ to lie in the spectrum of the action. We thus get a finite projective ${ }^{\circ} \mathscr{C}$-bimodule ${ }^{\circ} \mathscr{H}_{u}$. Computations similar to those in the above example, show that the left ${ }^{\circ} \mathcal{C}$-action is given by

$$
\left\langle x_{i}, c \cdot x_{j}\right\rangle=\sum_{r} c_{r, i}^{u *} c c_{r, j}^{u},
$$

where $c_{r}^{u}:=\left(c_{r j}^{u}\right)_{j} \in \bar{L}_{u}$ is an orthonormal basis of $\bar{L}_{u}$.

Remark. The restriction functor and the spectral functor of a quantum quotient define the same algebra ${ }^{\circ} \mathcal{C}$. However, the associated bimodules are different in general as, in the first case, they are free and never zero for a nonzero object, whilst in the second, non-spectral representations give zero bimodules.

8.4. The completed Hilbert bimodules $\mathscr{H}_{u}$. Consider ${ }^{\circ} \mathcal{C}$ with its maximal $\mathrm{C}^{*}$ norm, which is finite by [34]. Completing ${ }^{\circ} \mathscr{H}_{u}$ in the norm $\|\xi\|:=\|\langle\xi, \xi\rangle\|^{1 / 2}$, gives a right Hilbert module $\mathscr{H}_{u}$ over the completion $\mathcal{C}$ of ${ }^{\circ} \mathcal{C}$. There is an isometry $\mathscr{H}_{u} \rightarrow \tau_{u} \otimes \mathcal{C}$ extending the algebraic isometry $S_{z}$. Hence $\mathscr{H}_{u}$ is a finite projective right Hilbert $\mathcal{C}$-module. Consequently, every right module map on ${ }^{\circ} \mathcal{H}_{u}$ extends to an adjointable bounded map on $\mathscr{H}_{u}$. Hence the left ${ }^{\circ} \mathcal{C}$-action extends to a unital ${ }^{*}$-homomorphism $\mathcal{C} \rightarrow \mathscr{L}_{\mathcal{C}}\left(\mathscr{H}_{u}\right)$ thus making $\mathscr{H}_{u}$ into a Hilbert $\mathcal{C}$-bimodule.

To show that the left action is faithful we need norm continuity of the multiplication of bimodules. 


\section{Proposition 8.7. The multiplication map}

$$
\xi \otimes \xi^{\prime} \in{ }^{\circ} \mathscr{H}_{u} \otimes \circ e^{\circ} \mathscr{H}_{u^{\prime}} \rightarrow \xi \cdot \xi^{\prime} \in{ }^{\circ} \mathscr{H}_{u \otimes u^{\prime}}
$$

is an isometry of prehilbertian ${ }^{\circ} \mathcal{C}$-bimodules. Hence $\left\|\xi \cdot \xi^{\prime}\right\| \leq\|\xi\|\left\|\xi^{\prime}\right\|$.

Proof. Using a product solution of the conjugate equations,

$$
\begin{aligned}
\left\langle\xi \cdot \xi^{\prime}, \eta \cdot \eta^{\prime}\right\rangle & =\lambda\left(\mu\left(R_{u \otimes u^{\prime}}^{*}\right)\right) \xi^{*} \cdot \xi^{*} \cdot \eta \cdot \eta^{\prime} \\
& =\lambda\left(\mu\left(R_{u^{\prime}}^{*}\right) \circ \lambda\left(\mu\left(1_{\bar{u}^{\prime}} \otimes R_{u}^{*} \otimes 1_{u^{\prime}}\right)\right) \xi^{*} \cdot \xi^{*} \cdot \eta \cdot \eta^{\prime}\right. \\
& =\left(\lambda\left(\mu\left(R_{u^{\prime}}^{*}\right)\right) \xi^{\prime *}\right) \cdot\left(\lambda\left(\mu\left(R_{u}^{*}\right)\right) \xi^{*} \cdot \eta\right) \cdot \eta^{\prime} \\
& =\left\langle\xi^{\prime},\langle\xi, \eta\rangle \cdot \eta^{\prime}\right\rangle \\
& =\left\langle\xi \otimes \xi^{\prime}, \eta \otimes \eta^{\prime}\right\rangle,
\end{aligned}
$$

as required.

Consequently, · extends to an associative multiplication $\xi \cdot \xi^{\prime}$ on the completed bimodules $\mathscr{H}_{u}$ and $\mathscr{H}_{u^{\prime}}$.

Proposition 8.8. The extended left action of $\mathcal{C}$ on $\mathscr{H}_{u}$ is faithful whenever $1_{\mu_{u}} \neq 0$.

Proof. If $c \cdot \xi=0$ for all $\xi \in \mathscr{H}_{u}$, then

$$
c \cdot\left(\lambda\left(\mu\left(\bar{R}_{u}^{*}\right)\right) \xi \cdot \eta\right)=\lambda\left(\mu\left(\bar{R}_{u}^{*}\right)\right) c \cdot \xi \cdot \eta=0
$$

for all $\eta \in \mathscr{H}_{\bar{u}}$. On the other hand, $\lambda\left(\mu\left(\bar{R}_{u}^{*}\right)\right) \xi \cdot \eta=\left\langle\xi^{*}, \eta\right\rangle$, and these coefficients $\operatorname{span}^{\circ} \mathcal{C}$ if $1_{\mu_{\bar{u}}} \neq 0$, i.e., if $1_{\mu_{u}} \neq 0$.

8.5. Quantum group representations on $\mathscr{H}_{\boldsymbol{u}}$. We next construct quantum group representations on the bimodules $\mathscr{H}_{u}$. Let $G_{\tau}$ denote, as before, the Woronowicz dual of $\tau: \mathcal{A} \rightarrow$ Hilb.

Proposition 8.9. Given an object $u$ of $\mathcal{A}$, there is a unique bimodule representation $\operatorname{Ind}\left(\mu_{u}\right)$ of $G_{\tau}$ on $\mathscr{H}_{u}$ such that

$$
\operatorname{Ind}\left(\mu_{u}\right)(M \otimes \psi)=M \otimes \hat{v}(\psi),
$$

$M \in\left(\mu_{v}, \mu_{u}\right), \psi \in\left(\iota, \tau_{v}\right)$. Ind $\left(\mu_{u}\right)$ is a full bimodule representation.

Proof. The relation between the invertible antilinear maps $j_{v}: \tau_{v} \rightarrow \tau_{\bar{v}}$ and the coefficients of the corresponding representations of $G_{\tau}$ is given by $\hat{\bar{v}} j_{v} \psi=\sum \phi_{i} \otimes$ $\hat{v}_{j_{v}^{*} \phi_{i}, \psi}^{*}$, where $\phi_{i}$ an orthonormal basis of $\tau_{\bar{v}}$. This relation together with (7.1) allows us to verify (3.1). (3.2), (3.3) and (3.5) follow from straightforward computations, whilst (3.4) is a consequence of the corresponding relation for the Hilbert space representation $\hat{v}$. We show that $\operatorname{Ind}\left(\mu_{u}\right)$ is a full representation. A $G_{\tau}$-fixed vector 
$\xi$ in $\mathscr{H}_{u}$ for the underlying module representation is a simple tensor of the form $\xi=T \otimes 1_{\iota}, T \in\left(\iota, \mu_{u}\right)$. For any $c \in \mathcal{C}$ of the form $c=Y \otimes \psi, Y \in\left(\mu_{v}, \iota\right)$, $\psi \in \tau_{v}$, we have

$$
\begin{aligned}
\xi \cdot c & =(T \otimes Y) \otimes \psi \\
& =\left(T \otimes 1_{\iota} \circ 1_{\iota} \otimes Y\right) \otimes \psi \\
& =(T \circ Y) \otimes \psi \\
& =\left(1_{\iota} \otimes T \circ Y \otimes 1_{\iota}\right) \otimes \psi \\
& =(Y \otimes T) \otimes \psi \\
& =c \cdot \xi .
\end{aligned}
$$

Proposition 8.10. For any arrow $X \in\left(\mu_{u}, \mu_{u^{\prime}}\right)$, the norm continuous extension of $\lambda(X)$ to the completed Hilbert modules lies in the arrow space $\left(\operatorname{Ind}\left(\mu_{u}\right), \operatorname{Ind}\left(\mu_{u^{\prime}}\right)\right)$ of $\operatorname{Bimod}_{\alpha}\left(G_{\tau}\right)$.

Proof. Property (7.1) shows that $\lambda$ is a ${ }^{*}$-functor from the $\mathrm{C}^{*}$-category $\mathcal{M}_{\mu}$ to the category of prehilbertian ${ }^{\circ} \mathcal{C}$-bimodules. Thus $\lambda(X)$ is bounded and hence extends uniquely to a bimodule map between the completed Hilbert bimodules. On the other hand, the obvious commutation relations between $\lambda(X)$ and the action of $G_{\tau}$ imply that $\lambda(X)$ is an intertwining operator between the corresponding bimodule representations of $G_{\tau}$.

8.6. The induction functor Ind: $\mathcal{M}_{\mu} \rightarrow \operatorname{Bimod}_{\alpha}\left(G_{\tau}\right)$. We may thus define a ${ }^{*}$-functor of $\mathrm{C}^{*}$-categories,

$$
\text { Ind: } \mathcal{M}_{\mu} \rightarrow \operatorname{Bimod}_{\alpha}\left(G_{\tau}\right),
$$

taking an object $\mu_{u}$ to $\operatorname{Ind}\left(\mu_{u}\right)$ and an arrow $X \in\left(\mu_{u}, \mu_{u^{\prime}}\right)$ to the extension of $\lambda(X)$. This is the induction functor.

8.7. The natural transformation $S$ and the generalized Swan's theorem. The maps $S_{u}$ defined in Section 8.1 extend uniquely to isometries $S_{u}: \mathscr{H}_{u} \rightarrow \tau_{u} \otimes \mathcal{C}$ making the following diagrams commute for $A \in\left(u, u^{\prime}\right)$,

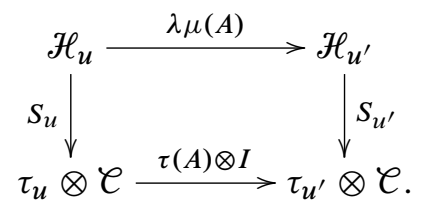

Proposition 8.11. $S_{u} \in\left(\operatorname{Ind}\left(\mu_{u}\right), \hat{u} \otimes \alpha\right)$ in the category $\operatorname{Mod}_{\alpha}\left(G_{\tau}\right)$. 
Proof. For $\xi=M \otimes \psi_{i}$, with $M \in\left(\mu_{v}, \mu_{u}\right),\left(\psi_{j}\right)$ an orthonormal basis of $\tau_{v}$, and orthonormal bases $\left(\phi_{r}\right)$ and $\left(\eta_{p}\right)$ of $\tau_{u}$ and $\tau_{\bar{u}}$ respectively,

$$
\begin{aligned}
\hat{u} \otimes \alpha & \circ S_{u}\left(M \otimes \psi_{i}\right) \\
= & \sum_{r} \hat{u} \otimes \alpha\left(\phi_{r} \otimes L\left(\phi_{r}\right)^{*}\left(M \otimes \psi_{i}\right)\right) \\
= & \sum_{r} \hat{u} \otimes \alpha\left(\phi_{r} \otimes\left(\mu\left(R_{u}^{*}\right) \circ \tilde{\mu}_{\bar{u}, u} \circ 1_{\mu_{\bar{u}}} \otimes M \circ \tilde{\mu}_{\bar{u}, v}^{*}\right) \otimes\left(j_{u} \phi_{r} \otimes \psi_{i}\right)\right) \\
= & \sum_{r, s, p, j} \phi_{s} \otimes\left(\mu\left(R_{u}^{*}\right) \circ \tilde{\mu}_{\bar{u}, u} \circ 1_{\mu_{\bar{u}}} \otimes M \circ \tilde{\mu}_{\bar{u}, v}^{*}\right)
\end{aligned}
$$

Remark. The map $u \rightarrow S_{u}$ is a natural transformation from Ind $\mu$ to $\tau \otimes 1$ taking values in $\operatorname{Mod}_{\alpha}\left(G_{\tau}\right)$.

\section{Extending Ind to a full tensor functor}

As in the previous sections, we consider a pair of ${ }^{*}$-functors between tensor $\mathrm{C}^{*}$ categories, $\tau: \mathcal{A} \rightarrow$ Hilb and $\mu: \mathcal{A} \rightarrow \mathcal{M}, \tau$ is tensorial and $\mu$ quasi-tensorial, and $\mathcal{A}$ has conjugates.

Now $\mathcal{M}_{\mu}$ is a $C^{*}$-category, but not a tensor $C^{*}$-category in general. This suggests looking for an extension of Ind to $\mathcal{M}_{\mu}^{\otimes}$. Here we show that the full tensor subcategory of $\operatorname{Bimod}_{\alpha}\left(G_{\tau}\right)$ with objects $\operatorname{Ind}\left(\mu_{u}\right)$ admits a natural realization as a strict tensor $\mathrm{C}^{*}$-category $\mathcal{T}$ and that Ind extends uniquely to a strict tensor isomorphism between $\mathcal{M}_{\mu}^{\otimes}$ and $\mathcal{T}$.

In the next subsection we construct new bimodules $\mathscr{H}_{u}$ associated with finite sequences $\underline{u}=\left(u_{1}, \ldots, u_{n}\right)$ of objects of $\mathcal{A}$. This construction reduces to that of the bimodules $\mathscr{H}_{u}$ of Sections 7 and 8 for sequences of length 1 .

If $\underline{u}=\left(u_{1}, \ldots, u_{n}\right)$ is such a sequence and if $\bar{u}_{i}$ is a conjugate of $u_{i}$, we write $\underline{u}$ for $\left(\bar{u}_{n}, \ldots, \bar{u}_{1}\right)$. If $R_{u_{i}}, \bar{R}_{u_{i}}$ is a solution of the conjugate equations for $u_{i}$, we denote by $R_{\underline{u}}$ and $\bar{R}_{\underline{u}}$ the solutions of the conjugate equations for $u_{1} \otimes \cdots \otimes u_{n}$ given by the product formula. Similarly, starting with the image solutions $\hat{R}_{u_{i}}:=\tilde{\mu}_{\bar{u}, u}^{*} \circ \mu\left(R_{u}\right)$ for $\mu_{u_{i}}$ in $\mathcal{M}$, we use the product formula to define the solution $\widehat{R}_{\underline{u}}$ for $\mu_{u_{1}} \otimes \cdots \otimes \mu_{u_{n}}$. 
Lemma 9.1. $\hat{R}_{\underline{u}}=\tilde{\mu}_{\underline{\underline{u}}, \underline{u}}^{*} \circ \mu\left(R_{\underline{u}}\right)$.

Proof. We prove the lemma by induction on the length of $\underline{u}$. The result holds by definition if this length is one. Suppose that $\underline{u}=(\underline{v}, w)$. Then, by construction,

$$
\widehat{R}_{\underline{u}}=1_{\mu_{\bar{w}}} \otimes \hat{R}_{\underline{v}} \otimes 1_{\mu_{w}} \circ \widehat{R}_{w} .
$$

Hence by the induction hypothesis,

$$
\widehat{R}_{\underline{u}}=1_{\mu_{\bar{w}}} \otimes \tilde{\mu}_{\underline{\underline{v}}, \underline{v}}^{*} \otimes 1_{\mu_{w}} \circ 1_{\mu_{\bar{w}}} \otimes \mu\left(R_{\underline{v}}\right) \otimes 1_{\mu_{w}} \circ \tilde{\mu}_{\bar{w}, w}^{*} \circ \mu\left(R_{w}\right) .
$$

But, by naturality,

$$
1_{\mu_{\bar{w}}} \otimes \mu\left(R_{\underline{v}}\right) \otimes 1_{\mu_{w}} \circ \tilde{\mu}_{\bar{w}, w}^{*}=\tilde{\mu}_{\bar{w}, z, w}^{*} \circ \mu\left(1_{\bar{w}} \otimes R_{\underline{v}} \otimes 1_{w}\right),
$$

where $z=\bar{v}_{n} \otimes \cdots \otimes \bar{v}_{1} \otimes v_{1} \otimes \cdots \otimes v_{n}$ if $\underline{v}=\left(v_{1}, \ldots, v_{n}\right)$. On the other hand, the following relation follows easily from associativity of $\mu$,

$$
1_{\mu_{\bar{w}}} \otimes \tilde{\mu}_{\underline{\underline{v}}, \underline{v}}^{*} \otimes 1_{\mu_{w}} \circ \tilde{\mu}_{\bar{w}, z, w}^{*}=\tilde{\mu}_{\underline{u}, \underline{u}}^{*},
$$

thus completing the proof.

9.1. New Hilbert bimodules $\mathscr{H}_{\underline{u}}$. Let $\underline{u}=\left(u_{1}, \ldots, u_{n}\right)$ be a finite sequence of objects of $\mathcal{A}$ and set

$$
\mu_{\underline{u}}:=\mu_{u_{1}} \otimes \cdots \otimes \mu_{u_{n}} .
$$

Let ${ }^{\circ} \mathcal{H}_{\underline{u}}$ be the linear space $\sum_{v}\left(\mu_{v}, \mu_{\underline{u}}\right) \otimes \tau_{v}$, the sum being taken over the objects of $\mathcal{A}$, quotiented by the linear subspace generated by elements of the form $M \circ \mu(A) \otimes$ $T-M \otimes \tau(A) \circ T$.

We proceed as in the construction of the bimodules ${ }^{\circ} \mathcal{H}_{u}$ of Section 7 , defining successively multiplication, the functor $\lambda$, adjoint and sesquilinear form.

Define bilinear maps ${ }^{\circ} \mathcal{H}_{\underline{u}} \times{ }^{\circ} \mathcal{H}_{\underline{u}^{\prime}} \rightarrow{ }^{\circ} \mathcal{H}_{\underline{u}, \underline{u}^{\prime}}$. For $\xi=L \otimes \psi, \eta=M \otimes \phi$, where $L \in\left(\mu_{w}, \mu_{\underline{u}}\right), M \in\left(\mu_{v}, \mu_{\underline{u}^{\prime}}\right), \psi \in \tau_{w}, \phi \in \tau_{v}$, set

$$
\xi \eta:=(L \otimes M) \circ \tilde{\mu}_{w, v}^{*} \otimes(\psi \otimes \phi) .
$$

It is easy to check that these maps are well defined and associative.

For a reason that will soon become clear, this new multiplication does not coincide with the multiplication $\xi \cdot \eta$ of Section 7 if $\underline{u}$ or $\underline{u}^{\prime}$ are objects of $\mathcal{A}$. We have therefore used a different notation. However, the two multiplications coincide if $\underline{u}$ or $\underline{u}^{\prime}$ are the tensor unit $\iota$, as $\tilde{\mu}_{\iota, \underline{u}}=\tilde{\mu}_{\underline{u}, \iota}=1_{\mu_{\underline{u}}}$. Hence ${ }^{\circ} \mathcal{H}_{(\iota)}$ is again the algebra ${ }^{\circ} \ell$ and ${ }^{\circ} \mathcal{H}_{\underline{u}}$ is a ${ }^{\circ} \mathcal{C}$-bimodule. Furthermore, as a bimodule, we do have ${ }^{\circ} \mathcal{H}_{u}={ }^{\circ} \mathcal{H}_{u}$ if $u=(u)$.

Given $Y \in\left(\mu_{\underline{u}}, \mu_{\underline{u}^{\prime}}\right)$ a bimodule map, $\lambda(Y):{ }^{\circ} \mathscr{H}_{\underline{u}} \rightarrow^{\circ}{ }^{\circ} \mathcal{H}_{\underline{u}^{\prime}}$ is defined in the obvious way. $\lambda$ is a covariant functor from $\mathcal{M}_{\mu}^{\otimes}$ to the category of ${ }^{\circ} \mathcal{C}$-bimodules. In particular, if $\underline{u}=\left(u_{1}, \ldots, u_{n}\right)$,

$$
\lambda\left(\tilde{\mu}_{\underline{u}}\right):{ }^{\circ} \mathcal{H}_{\underline{u}} \rightarrow^{\circ} \mathscr{H}_{u_{1} \otimes \cdots \otimes u_{n}}
$$


relates the new and old bimodules. Moreover, the respective multiplications are related by

$$
\lambda\left(\tilde{\mu}_{\underline{u}, \underline{u}^{\prime}}\right)(\xi \eta)=\left(\lambda\left(\tilde{\mu}_{\underline{u}}\right) \xi\right) \cdot\left(\lambda\left(\tilde{\mu}_{\underline{\underline{u}}^{\prime}}\right) \eta\right)
$$

for $\xi \in{ }^{\circ} \mathcal{H}_{\underline{u}}, \eta \in{ }^{\circ} \mathscr{H}_{\underline{u}^{\prime}}$. In particular

$$
\lambda\left(\tilde{\mu}_{u, v}\right) \xi \eta=\xi \cdot \eta, \quad \xi \in{ }^{\circ} \mathscr{H}_{u}, \eta \in{ }^{\circ} \mathscr{H}_{v} .
$$

The adjoint $*{ }^{\circ} \mathscr{H}_{\underline{u}} \rightarrow{ }^{\circ} \mathscr{H}_{\underline{\bar{u}}}$ is defined as before by

$$
(M \otimes \psi)^{*}:=M^{\bullet} \otimes j_{v} \psi
$$

but where ${ }^{-}$now refers to $\widehat{R}_{\underline{u}}$. The adjoint is well defined and independent of the choice of solutions of the conjugate equations for $v$. However, if we change the solution of the conjugate equations for $\underline{u}$ using a sequence of invertibles $\left(X_{1}, \ldots, X_{n}\right)$ wth $X_{i} \in\left(\bar{u}_{i}, \tilde{u}_{i}\right),(M \otimes \psi)^{*}$ becomes $\left(\mu\left(X_{1}\right) \otimes \cdots \otimes \mu\left(X_{n}\right) \circ M^{\bullet}\right) \otimes j_{v} \psi$.

Nevertheless $*$ is an antilinear map satisfying the properties of Proposition 7.1 if we use tensor product and conjugate solutions.

The ${ }^{\circ} \mathcal{C}$-valued form on ${ }^{\circ} \mathscr{H}_{\underline{u}}$ is defined by

$$
\left\langle\xi, \xi^{\prime}\right\rangle:=\lambda\left(\widehat{R}_{\underline{u}}^{*}\right)\left(\xi^{*} \xi^{\prime}\right) .
$$

One can easily check that a formula similar to (7.1) holds,

$$
\left\langle\xi, \xi^{\prime}\right\rangle=\left(\hat{R}_{v}^{*} \circ 1_{\mu_{\bar{v}}} \otimes\left(M^{*} \circ M^{\prime}\right) \circ \tilde{\mu}_{\bar{v}, v^{\prime}}^{*}\right) \otimes j_{v} \psi \otimes \psi^{\prime},
$$

hence the form reduces to that of ${ }^{\circ} \mathcal{H}_{u}$ if $\underline{u}=(u)$. As before, this form is independent of the choice of the conjugate of $\underline{u}$ in view of how $\xi^{*}$ changes and of Lemma 9.1. The above expression shows that $\lambda$ is a ${ }^{*}$-functor.

Since $\tilde{\mu}_{\underline{u}}$ is an isometry, (9.1) shows that $\lambda\left(\tilde{\mu}_{\underline{u}}\right)$ preserves the corresponding forms:

$$
\left\langle\lambda\left(\tilde{\mu}_{\underline{u}}\right) \xi, \lambda\left(\tilde{\mu}_{\underline{u}}\right) \xi^{\prime}\right\rangle=\left\langle\xi, \xi^{\prime}\right\rangle, \quad \xi, \xi^{\prime} \in{ }^{\circ} \mathscr{H}_{\underline{u}},
$$

generalizing Proposition 8.7. On the other hand, ${ }^{\circ} \mathcal{H}_{u_{1} \otimes \cdots \otimes u_{n}}$ is a finite projective prehilbertian bimodule, so the same is true of ${ }^{\circ} \mathcal{H}_{\underline{u}}$.

Completing ${ }^{\circ} \mathcal{H}_{u}$ in the norm derived from the maximal $\mathrm{C}^{*}$-norm of ${ }^{\circ} \mathcal{C}$ yields a Hilbert $\mathcal{C}_{\text {-bimodule }} \mathscr{H}_{\underline{u}}$. $\lambda$ extends to a ${ }^{*}$-functor from $\mathcal{M}_{\mu}^{\otimes}$ to the $\mathrm{C}^{*}$-category of

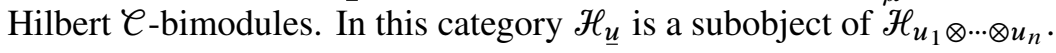

We next regard the associative multiplication $\xi, \eta \rightarrow \xi \eta$ as a bimodule map defined on the bimodule tensor product ${ }^{\circ} \mathcal{H}_{\underline{u}} \otimes \circ e^{\circ} \mathscr{H}_{\underline{u}^{\prime}} \rightarrow^{\circ} \mathscr{H}_{\underline{u}, \underline{u}^{\prime}}$.

Theorem 9.2. The multiplication $\xi \otimes \eta \rightarrow \xi \eta$ extends uniquely to a unitary map between Hilbert bimodules

$$
\xi \otimes \eta \in \mathscr{H}_{\underline{u}} \otimes \mathcal{e} \mathscr{H}_{\underline{u}^{\prime}} \rightarrow \xi \eta \in \mathscr{H}_{\underline{u}, \underline{u}^{\prime}}
$$


Proof. Using successively that $\lambda\left(\tilde{\mu}_{\underline{u}}\right)$ is isometric, Proposition 8.7, and relation (9.1), we conclude that multiplication is a densely defined isometry, extending to an isometry of the completions. To complete the proof it suffices to show that the set of all $\xi \eta$, with $\xi \in{ }^{\circ} \mathscr{H}_{\underline{u}}, \eta \in{ }^{\circ} \mathscr{H}_{\underline{u}^{\prime}} \operatorname{span}^{\circ} \mathcal{H}_{\underline{u}, \underline{u}^{\prime}}$.

Since multiplication is associative, it suffices to choose $\underline{u}$ to be a sequence $(u)$ consisting of a single element. Consider an element of ${ }^{\circ} \mathcal{H}_{u, u^{\prime}}$ of the form $M \otimes \psi$, where $M \in\left(\mu_{v}, \mu_{u} \otimes \mu_{\underline{u}^{\prime}}\right)$ and $\psi \in \tau_{v}$. Using the explicit linear isomorphism $\left(\mu_{v}, \mu_{u} \otimes \mu_{\underline{u}^{\prime}}\right) \simeq\left(\mu_{\bar{u}} \otimes \mu_{v}, \mu_{\underline{u}^{\prime}}\right)$, we may write $M=1_{\mu_{u}} \otimes T \circ \hat{\bar{R}}_{u} \otimes 1_{\mu_{v}}$ where $T \in\left(\mu_{\bar{u}} \otimes \mu_{v}, \mu_{\underline{u}^{\prime}}\right)$. We may also write $T=T^{\prime} \circ \tilde{\mu}_{\bar{u}, v}$, with $T^{\prime} \in\left(\mu_{\bar{u} \otimes v}, \mu_{\underline{u}^{\prime}}\right)$. Hence

$$
\begin{aligned}
M & =1_{\mu_{u}} \otimes\left(T^{\prime} \circ \tilde{\mu}_{\bar{u}, v}\right) \circ \hat{\bar{R}}_{u} \otimes 1_{\mu_{v}} \\
& =1_{\mu_{u}} \otimes T^{\prime} \circ 1_{\mu_{u}} \otimes \tilde{\mu}_{\bar{u}, v} \circ \tilde{\mu}_{u, \bar{u}}^{*} \otimes 1_{\mu_{v}} \circ \mu\left(\bar{R}_{u}\right) \otimes 1_{\mu_{v}} \\
& =1_{\mu_{u}} \otimes T^{\prime} \circ \tilde{\mu}_{u, \bar{u} \otimes v}^{*} \circ \tilde{\mu}_{u \otimes \bar{u}, v} \circ \mu\left(\bar{R}_{u}\right) \otimes 1_{\mu_{v}} \\
& =1_{\mu_{u}} \otimes T^{\prime} \circ \tilde{\mu}_{u, \bar{u} \otimes v}^{*} \circ \mu\left(\bar{R}_{u} \otimes 1_{v}\right) .
\end{aligned}
$$

Substituting this into $M \otimes \psi$ gives

$$
M \otimes \psi=\left(1_{\mu_{u}} \otimes T^{\prime} \circ \tilde{\mu}_{u, \bar{u} \otimes v}^{*}\right) \otimes\left(\tau\left(\bar{R}_{u}\right) \otimes \psi\right) .
$$

Writing $\tau\left(\bar{R}_{u}\right)=\sum_{j} \phi_{j} \otimes j_{u} \phi_{j}$, for an orthonormal basis $\left(\phi_{j}\right)$ of $\tau_{u}$, gives

$$
M \otimes \psi=\sum_{j} \xi_{j} \eta_{j}, \quad \xi_{j}=1_{\mu_{u}} \otimes \phi_{j} \in{ }^{\circ} \mathcal{H}_{u}, \quad \eta_{j}=T^{\prime} \otimes\left(j_{u} \phi_{j} \otimes \psi\right) \in{ }^{\circ} \mathcal{H}_{\underline{u}^{\prime}} .
$$

On one hand, as the multiplication maps are isometric, $\mathscr{H}_{\underline{u}} \mathscr{H}_{\underline{u}^{\prime}}$ realizes the tensor product of Hilbert bimodules. It has the virtue of being strictly associative, as so are the multiplication maps. We replace the original tensor product of Hilbert bimodules by this strictly associative tensor product. On the other hand, since the multiplication maps are unitary, we have tensor product decompositions,

$$
\mathscr{H}_{\underline{u}}=\mathscr{H}_{u_{1}} \ldots \mathscr{H}_{u_{n}},
$$

for $\underline{u}=\left(u_{1}, \ldots, u_{n}\right)$. (Note that the right-hand side is already norm closed, by finite projectivity.) In particular, if $1_{\mu_{u_{i}}} \neq 0$ for all $i, \mathscr{H}_{\underline{u}}$ is a full right Hilbert module with a faithful right $\mathcal{C}$-action. We thus have the following result.

Theorem 9.3. $\lambda$ is a strict tensor ${ }^{*}$-functor from $\mathcal{M}_{\mu}^{\otimes}$ to the tensor $C^{*}$-category of Hilbert $\mathcal{C}$-bimodules (with a strictly associative tensor product).

\section{2. $G_{\tau}$-representations on the bimodules $\mathscr{H}_{\underline{u}}$}

Proposition 9.4. Given a finite sequence $\underline{u}=\left(u_{1}, \ldots, u_{n}\right)$ of objects of $\mathcal{A}$, there is a unique bimodule representation $\operatorname{Ind}\left(\mu_{\underline{u}}\right)$ of $G_{\tau}$ on $\mathscr{H}_{\underline{u}}$ such that, for $M \in\left(\mu_{v}, \mu_{\underline{u}}\right)$, $\psi \in \tau_{v}$,

$$
\operatorname{Ind}\left(\mu_{\underline{u}}\right)(M \otimes \psi)=M \otimes \hat{v} \psi
$$


$\operatorname{Ind}\left(\mu_{\underline{u}}\right)$ is a full bimodule representation. Under the tensor product decomposition $\mathscr{H}_{\underline{u}}=\mathscr{H}_{u_{1}} \ldots \mathscr{H}_{u_{n}}, \operatorname{Ind}\left(\mu_{\underline{u}}\right)=\operatorname{Ind}\left(\mu_{u_{1}}\right) \otimes \cdots \otimes \operatorname{Ind}\left(\mu_{u_{n}}\right)$.

Proof. The only non-trivial statement is that $\operatorname{Ind}\left(\mu_{u}\right)$ is full. A $G_{\tau}$-fixed vector $\mathscr{H}_{u}$ is of the form $M \otimes 1_{\iota}$, with $M \in\left(\iota, \mu_{u}\right)$. As for the old modules, Proposition 8.9, one shows this element to be central.

As before, $\lambda(X)$ intertwines the representations $\operatorname{Ind}\left(\mu_{\underline{u}}\right)$ and $\left.\operatorname{Ind}\left(\mu_{\underline{u}^{\prime}}\right)\right)$ for $X \in$ $\left(\mu_{\underline{u}}, \mu_{\underline{u}^{\prime}}\right)$. We therefore have a strict tensor functor

$$
\text { Ind: } \mathcal{M}_{\mu}^{\otimes} \rightarrow \operatorname{Bimod}_{\alpha}\left(G_{\tau}\right) \text {, }
$$

the unique tensor extension of the functor Ind defined on $\mathcal{M}_{\mu}$ of the previous section.

We are now ready to state a central result of this paper, a version of the Frobenius reciprocity theorem 4.1 for quasi-tensor functors.

Theorem 9.5. Ind is a full and faithful strict tensor functor from $\mathcal{M}_{\mu}^{\otimes}$ to the category of bimodule representations of $G_{\tau}$ if the latter is endowed with a strictly associative tensor product.

Proof. $\mathcal{M}$ is a tensor $\mathrm{C}^{*}$-category with conjugates and Ind a relaxed tensor functor, hence automatically faithful [35]. It remains to show that Ind is full. This follows from the linear isomorphisms $\gamma:\left(\mu_{\underline{\underline{u}}}, \mu_{\underline{u}^{\prime}}\right) \rightarrow\left(\iota, \mu_{\underline{u}^{\prime}, \underline{\underline{u}}}\right), T \rightarrow X=T \otimes 1_{\mu_{\underline{\underline{u}}}} \circ \hat{\bar{R}}_{\underline{u}}$, and $\delta:\left(\operatorname{Ind}\left(\mu_{\underline{u}}\right), \operatorname{Ind}\left(\mu_{\underline{u}^{\prime}}\right)\right) \rightarrow\left(\iota, \operatorname{Ind}\left(\mu_{\underline{u}^{\prime}, \bar{u}}\right)\right)$, defined similarly, where $\mu$ is replaced by the quasi-tensor functor $\operatorname{Ind} \mu$. Hence any intertwiner in $\left(\operatorname{Ind}\left(\mu_{\underline{u}}\right), \operatorname{Ind}\left(\mu_{\underline{u}^{\prime}}\right)\right)$ is determined by a fixed vector in $\mathscr{H}_{\underline{u}^{\prime}, \bar{u}}$, which we already know to arise from an intertwiner in $\left(\iota, \mu_{\underline{u}^{\prime}, \bar{u}}\right)$, hence lying in the image of Ind.

Remark. The last proof uses only the functor of tensoring on the right by an identity arrow. This also makes sense for module intertwiners and hence shows the following result.

Theorem 9.6. Any module intertwiner from $\operatorname{Ind}\left(\mu_{\underline{u}}\right)$ to $\operatorname{Ind}\left(\mu_{u^{\prime}}\right)$, namely an intertwiner in the $C^{*}$-category $\operatorname{Mod}_{\alpha}\left(G_{\tau}\right)$, is automatically a bimodule intertwiner.

9.3. The functor $\operatorname{Ind} \mu: \mathcal{A} \rightarrow \operatorname{Bimod}_{\alpha}\left(G_{\tau}\right)$. We finally define the composed functor

$$
\operatorname{Ind} \mu: \mathcal{A} \rightarrow \operatorname{Bimod}_{\alpha}\left(G_{\tau}\right) .
$$

Theorem 9.7. If ( $\mu, \tilde{\mu})$ is a quasi-tensor (relaxed tensor, tensor) functor, $\operatorname{Ind} \mu$ is a quasi-tensor (relaxed tensor, tensor) functor too, with natural transformation $\operatorname{Ind}(\mu)$ given by the --multiplication maps,

$$
\operatorname{Ind}\left(\tilde{\mu}_{u, u^{\prime}}\right): \xi \xi^{\prime} \in \mathscr{H}_{u} \mathscr{H}_{u^{\prime}} \rightarrow \xi \cdot \xi^{\prime} \in \mathscr{H}_{u \otimes u^{\prime}} .
$$

Furthermore, Ind is tensor isomorphism from $(\mu, \tilde{\mu})$ to $(\operatorname{Ind} \mu, \widetilde{\operatorname{Ind} \mu})$. 
Proof. Since Ind is a strict tensor functor and $\mu$ is quasi-tensor (relaxed tensor, tensor, respectively), their composition Ind $\mu$, with the composed natural transformation, is quasi-tensor (relaxed tensor, tensor, resp.); see Section 2.2. This natural transformation is precisely the map $\xi \xi^{\prime} \rightarrow \xi \cdot \xi^{\prime}$. The last statement is clear.

We next prove Theorems 6.5 and 6.6.

Proof of Theorems 6.5 and 6.6. We briefly recall from [35] how to get a pair of functors $\mu$ and $\tau$. Assume that $\mathcal{M}$ has conjugates and an irreducible tensor unit $\iota$, and fix an object $x$ in $\mathcal{M}$ with intrinsic dimension $>1$ and a standard solution $R, \bar{R}$ of the conjugate equations for $x$. By Jones's result [18] the intrinsic dimension of $x$ can only take the values $d=2 \cos \frac{\pi}{\ell}$, for $\ell=3,4, \ldots$ or $d \geq 2$. Consider the universal tensor *-category $\mathcal{T}_{d}$ with objects the finite words in $u$ and $\bar{u}$ and whose arrows are generated by two arrows $S \in(\iota, \bar{u} \otimes u)$ and $\bar{S} \in(\iota, u \otimes \bar{u})$ subject to the relations expressing $(S, \bar{S})$ as a normalized solution of the conjugate equations for $u$. $\iota$ is the empty word and acts as a tensor unit. $\mathcal{T}_{d}$ is a tensor $\mathrm{C}^{*}$-category for the allowed values of $d$. Furthermore there is a tensor functor $\tau$ from $\mathcal{T}_{d}$ to the category of Hilbert spaces if and only if $d \geq 2$, and all such functors can be easily classified. Picking an embedding $\tau$, we get an associated compact quantum group $G_{\tau}=A_{u}(F)$, where $F$ is an invertible matrix such that $\operatorname{Tr}\left(F F^{*}\right)=\operatorname{Tr}\left(\left(F F^{*}\right)^{-1}\right)=R^{*} R$. Furthermore we have a canonical tensor functor $\mu: \mathcal{T}_{d} \rightarrow \mathcal{M}$ such that $\mu(u)=x, \mu(\bar{u})=\bar{x}$, $\mu(S)=R, \mu(\bar{S})=\bar{R}$, and we may now apply our main result.

Similarly, given a real or pseudoreal solution of the conjugate equations in $\mathcal{M}$, namely $R \in\left(\iota, x^{2}\right)$ with $R^{*} \otimes 1_{x} \circ 1_{x} \otimes R= \pm 1_{x}$, we consider the associated universal Temperley-Lieb categories $\mathcal{T}_{\text {rd }}$ and $\mathcal{T}_{\text {pd }}$ with generating arrow $S \in\left(\iota, u^{2}\right)$. If $R^{*} \circ R \geq 2$, a choice of an embedding of $\mathcal{T}_{\text {pd }}$ or $\mathcal{T}_{\text {rd }}$ ) into the Hilbert spaces provides a quantum group $A_{o}(F)$ with $F$ an invertible matrix satisfying $F \bar{F}= \pm I$, $\operatorname{Tr}\left(F F^{*}\right)=\operatorname{Tr}\left(\left(F F^{*}\right)^{-1}\right)=R^{*} \circ R$.

\section{An adjoint pair of functors}

Recall that a pair of functors $F: \Phi \rightarrow \Phi^{\prime}$ and $F^{\prime}: \Phi^{\prime} \rightarrow \Phi$ between categories is an adjoint pair if, for any pair of objects $\phi \in \Phi, \phi^{\prime} \in \Phi^{\prime}$, there is an isomorphism $\beta_{\phi^{\prime}, \phi}:\left(\phi^{\prime}, F_{\phi}\right) \rightarrow\left(F_{\phi^{\prime}}^{\prime}, \phi\right)$ natural in $\phi$ and $\phi^{\prime}$.

In this section we show that, essentially by construction, the pair (Ind, $\mu$ ) gives rise to an adjoint pair.

To this end, we assume as before that $\tau: \mathcal{A} \rightarrow$ Hilb is a tensor functor into the category of Hilbert spaces, so that $\mathcal{A}$ is a category of representations of a compact quantum group $G_{\tau}$, and that $\mu: \mathcal{A} \rightarrow \mathcal{M}$ is a quasi-tensor functor of strict tensor $\mathrm{C}^{*}$-categories with irreducible tensor units and construct the corresponding Hilbert C*-bimodules. 
Following Mackey's construction of the induced representation for locally compact groups, we consider the scalar-valued inner product on $\mathcal{H}_{u}$ given by composing

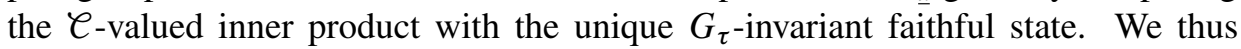
get a Hilbert space, $H_{u}$, and the bimodule representation $\operatorname{Ind}\left(\mu_{u}\right)$ of $G_{\tau}$ defines a densely defined representation of $G_{\tau}$ on $H_{u}$. This representation is isometric as the state is invariant and extends uniquely to a unitary representation of $G_{\tau}$ again denoted by $\operatorname{Ind}\left(\mu_{\underline{u}}\right)$. However, although we start with a finite-dimensional representation, the Hilbert space of the induced representation in general fails to be finite-dimensional, hence we need to work with the category of not necessarily finitedimensional unitary representations of $G_{\tau}$, denoted by $\widehat{\operatorname{Rep}}\left(G_{\tau}\right)$. We thus have a functor, Ind: $\mathcal{M}_{\mu}^{\otimes} \rightarrow \widetilde{\operatorname{Rep}}(G)$. We let $\widetilde{\mathcal{M}_{\mu}^{\otimes}}$ be the tensor $W^{*}$-category completion of $\mathcal{M}_{\mu}^{\otimes}$ under infinite direct sums, cf. [22]. Then $\mu$ and Ind extend uniquely to ${ }^{*}$-functors on $\widetilde{\operatorname{Rep}}(G)$ and $\widetilde{\mathcal{M}_{\mu}^{\otimes}}$, respectively.

Theorem 10.1. The pair offunctors Ind: $\widetilde{\mathcal{M}_{\mu}^{\otimes}} \rightarrow \widetilde{\operatorname{Rep}}\left(G_{\tau}\right)$ and $\mu: \widetilde{\operatorname{Rep}}\left(G_{\tau}\right) \rightarrow \widetilde{\mathcal{M}_{\mu}^{\otimes}}$ is an adjoint pair.

Proof. Note that the linear span of the images of elements of the form $T \otimes \psi$, where $T \in\left(\mu_{v}, \mu_{u}\right)$ and $\psi \in \tau_{v}$, where $v$ runs over the irreducible representations of $G_{\tau}$, is dense in $H_{\underline{u}}$. If we fix an irreducible $v$, the space of intertwiners $\left(v, \operatorname{Ind}\left(\mu_{\underline{u}}\right)\right)$ is given precisely by the set of maps $\widehat{T}: \psi \in \tau_{v} \rightarrow T \otimes \psi \in H_{\underline{u}}$, with $T \in\left(\mu_{v}, \mu_{\underline{u}}\right)$. Hence there is a linear isomorphism $\left(\mu_{v}, \mu_{\underline{u}}\right) \rightarrow\left(v, \operatorname{Ind}\left(\mu_{\underline{u}}\right)\right)$, natural in $\mu_{\underline{u}}$. This isomorphism extends uniquely to a linear isomorphism natural in $v$.

\section{Full bimodule representations from group actions}

Which ergodic actions $(\mathcal{C}, \alpha, G)$ can arise from a pair of functors Hilb $\stackrel{\tau}{\leftarrow} \mathcal{A} \stackrel{\mu}{\rightarrow} \mathcal{M}$ with $\mu$ relaxed tensor? In this section we attack this problem when $G_{\tau}$ is a compact group.

By Theorems 6.2 and 6.3, a necessary condition is that $u \otimes \alpha$ can be made into a full bimodule representation of $G$ for every $u \in \operatorname{Rep}(G)$. Using the results of [43], [15], given an ergodic action $(\mathcal{C}, \alpha)$ of $G$ we shall construct, a canonical full bimodule representation on every $G$-submodule $X_{u} \subset H_{u} \otimes \mathcal{C}$, where $u$ is an object of $\operatorname{Rep}(G)$. The submodule is the full module $H_{u} \otimes \mathcal{C}$ for all $u$ precisely when the multiplicity of $u$ is maximal.

It turns out that $u \rightarrow X_{u}$ is a quasi-tensor functor in general, related to the spectral functor of the ergodic action, and is relaxed tensor when the ergodic action is of full multiplicity.

We shall classify the full bimodule structures on the intermediate projective $G$ submodules $X_{u} \subset Y \subset u \otimes \alpha$ assuming that the weak completion of $\mathcal{C}$ in the 
GNS representation of the invariant trace is a finite type I von Neumann algebra, and hence induced by an ergodic action $\beta$ of a closed subgroup $K$ on a matrix algebra $\mathcal{F}$, cf. Section 2.3 (Theorem 11.9).

This provides an obstruction to the existence of full bimodule representation structures when multiplicities of the primitive action of $K$ are low but nonzero. More precisely, we shall show that certain low multiplicity actions as well as certain ergodic actions of SU(2) are excluded (Corollary 11.11, Example 11.10).

11.1. The minimal full bimodule representations. Let $(\mathcal{F}, \beta, K)$ be an ergodic action of a compact group $K$ on a unital $C^{*}$-algebra $\mathscr{F}$. Given an object $v$ of $\operatorname{Rep}(K)$, recall from Section 2 that the spectral space $\bar{L}_{v}$ is the complex conjugate of the set $L_{v}$ of all linear maps $T: H_{v} \rightarrow \mathcal{F}$ intertwining $v$ with $\beta$ and that, by the multiplicity bound theorem, Section 2.3, $\operatorname{dim}\left(\bar{L}_{v}\right) \leq \operatorname{dim}(v)$. If $v$ is irreducible, $\operatorname{dim}\left(\bar{L}_{v}\right)$ is the multiplicity of $v$ in $\beta$, denoted by mult $(v)$. Recall also that $\bar{L}_{v}$ is a Hilbert space with inner product $\langle\bar{S}, \bar{T}\rangle:=\sum_{i} S\left(\psi_{i}\right) T\left(\psi_{i}\right)^{*}$, where $\left(\psi_{i}\right)$ is an orthonormal basis of $H_{v}$.

If $\bar{L}_{v} \neq 0$, we construct a natural nonzero $K$-module subrepresentation of $v \otimes \beta$ with a full bimodule structure.

Consider the linear map $Z_{v}: \bar{T} \in \bar{L}_{v} \rightarrow \sum \psi_{i} \otimes T\left(\psi_{i}\right)^{*} \in H_{v} \otimes \mathcal{F}$, clearly independent of the choice of the orthonormal basis. The range of $Z_{v}$ is the space of $K$-fixed vectors in $H_{v} \otimes \mathcal{F}$ for the action $v \otimes \beta$. We may identify $Z_{v}$ with a rectangular matrix $\left(T_{k}\left(\psi_{i}\right)^{*}\right)$, where $\bar{T}_{k}$ is an orthonormal basis of $\bar{L}_{v}$. Since $\iota \otimes \beta_{k}\left(Z_{v}\right)=v(k)^{*} \otimes I Z_{v}, Z_{v}^{*}$ is referred to as the eigenmatrix of $\beta$ in [47].

Now, $Z_{v}$ takes the inner product of $\bar{L}_{v}$ to the inner product of the range inherited from $H_{v} \otimes \mathscr{F}$ as a right Hilbert $\mathscr{F}$-module, which on that subspace indeed takes values in $\mathbb{C}$ since $\beta$ is ergodic.

Extend $Z_{v}$ uniquely to an adjointable bounded map between Hilbert modules $\bar{L}_{v} \otimes \mathcal{F} \rightarrow H_{v} \otimes \mathcal{F}$, still denoted by $Z_{v}$. It is easy to verify that $Z_{v}$ is an isometry, $Z_{v}^{*} Z_{v}=I$, intertwining the module representations $\iota_{L_{v}} \otimes \beta$ and $v \otimes \beta$, where $\iota_{L_{v}}$ is the trivial representation of $K$ on the Hilbert space $\bar{L}_{v}$. We may clearly identify $\mathscr{L}_{\mathcal{F}}\left(\bar{L}_{v} \otimes \mathscr{F}, H_{v} \otimes \mathscr{F}\right) \simeq \mathscr{L}\left(\bar{L}_{v}, H_{v}\right) \otimes \mathscr{F}$. Note that $X_{v}:=Z_{v}\left(\bar{L}_{v} \otimes \mathcal{F}\right)$ is a projective $\mathcal{F}$-submodule of $H_{v} \otimes \mathcal{F}$.

Remark. An easy computation shows that, if $\tau$ is the unique invariant normalized tracial state of $\mathcal{F}$ and $\operatorname{Tr}$ is the non-normalized trace of $\mathscr{L}\left(H_{v}\right), \operatorname{Tr} \otimes \tau\left(E_{v}\right)=\operatorname{dim}\left(\bar{L}_{v}\right)$, where $E_{v}:=Z_{v} Z_{v}^{*}$. Hence $Z_{v}$ is a unitary if and only if $\operatorname{dim}\left(L_{v}\right)=\operatorname{dim}\left(H_{v}\right)$, i.e., $\bar{L}_{v}$ must have maximal dimension.

By the intertwining property of $Z_{v}, X_{v}$ is $K$-invariant. There is a faithful unital *-homomorphism

$$
\zeta: \mathcal{F} \rightarrow E_{v} \mathscr{L}\left(H_{v}\right) \otimes \mathscr{F} E_{v}, \quad \zeta(f)=Z_{v} I \otimes f Z_{v}^{*},
$$

making $X_{v}$ into a $\mathscr{F}$-bimodule. 
Proposition 11.1. For any representation $v$ of $K$ with $\bar{L}_{v} \neq 0, \zeta$ makes $X_{v}$ into a nonzero full bimodule $K$-representation, isomorphic to $\bar{L}_{v} \otimes \mathcal{F}$ via $Z_{v}$ with trivial left and right $\mathcal{F}$-actions, where $K$ acts as $\iota_{\bar{L}_{v}} \otimes \beta$.

Proof. By construction, $K$-fixed vectors are $\mathscr{F}$-central in $X_{v}$, as they correspond via $Z_{v}$ to the fixed vectors for ${ }^{l} \bar{L}_{v} \otimes \beta$, namely to $\bar{L}_{v} \otimes \mathbb{C}$, clearly central for the trivial bimodule action. Property (3.5) follows just as easily.

11.2. The intermediate full bimdule representations. Given $(\mathcal{F}, K, \beta)$ as before, we look for extensions of $X_{v}$ to full bimodule structures on intermediate projective $K$-module subrepresentations

$$
X_{v} \subset Y \subset H_{v} \otimes \mathscr{F} .
$$

Clearly, such submodules are the ranges of projections $E \in \mathscr{L}\left(H_{v}\right) \otimes \mathscr{F}$ satisfying

$$
E \geq E_{v},
$$

and the $K$-invariance condition

$$
\operatorname{Ad} v(k) \otimes \beta_{k}(E)=E, \quad k \in K .
$$

In what follows, we set $Z_{v}=0$ and $X_{v}=\{0\}$ if $\bar{L}_{v}=\{0\}$.

Proposition 11.2. Given $v \in \operatorname{Rep}(K)$ and a projection $E \in \mathscr{L}\left(H_{v}\right) \otimes \mathcal{F}$ satisfying (11.1) and (11.2), a unital ${ }^{*}$-homomorphism $\eta: \mathcal{F} \rightarrow E \mathscr{L}\left(H_{v}\right) \otimes \mathcal{F} E$ defines a full $K$-bimodule representation on $Y=E\left(H_{v} \otimes \mathcal{F}\right)$ if and only if

$$
\begin{gathered}
\eta\left(\beta_{k}(f)\right)=\operatorname{Ad} v(k) \otimes \beta_{k}(\eta(f)), \quad k \in K, \\
\eta(f) Z_{v}=Z_{v} I \otimes f, \quad f \in \mathcal{F} .
\end{gathered}
$$

Proof. The proof is straightforward. We just note that (11.3) corresponds to left $K$ equivariance in the sense of (3.5), whilst the property of being a full representation is expressed by (11.4), as for $X_{v}$.

Corollary 11.3. If $\bar{L}_{v}$ has maximal dimension, $v \otimes \beta$ becomes a full bimodule $K$-representation in a unique way.

Proof. By the previous remark, $Z_{v}$ is a unitary in $\mathscr{L}\left(H_{v}\right) \otimes \mathcal{F}$, and $X_{v}=H_{v} \otimes \mathcal{F}$. $\eta$ is uniquely determined by (11.4). This formula defines a ${ }^{*}$-homomorphism clearly satisfying (11.1)-(11.3) for $E=I$. 
11.3. Intermediate full bimodules for induced $\mathbf{C}^{*}$-actions. Now assume that $K$ is a closed subgroup of a compact group $G$ acting on $\mathcal{F}$, which may be either a $\mathrm{C}^{*}$ algebra or a von Neumann algebra. This action, $\beta$, is supposed to be continuous in the appropriate topology. Consider the induced algebra $\operatorname{Ind}(\mathscr{F})$ defined as in Section 2.3, the action $\rho$ of $G$ being given by right translation.

Let $v$ be a finite-dimensional unitary representation of $G$. In the next, known, proposition we determine the spectral spaces for the action $\rho$ in terms of those of the original action $\beta$.

Proposition 11.4. The map $T \in L_{v \uparrow_{K}}^{\beta} \rightarrow T^{\prime} \in L_{v}^{\rho}$, with $T^{\prime}: H_{v} \rightarrow \operatorname{Ind}(\mathcal{F})$ defined by $T^{\prime}(\psi)(g):=T(v(g) \psi)$, is unitary. As a consequence,

hence

$$
Z_{v}^{\rho}(g)=v(g)^{*} \otimes I Z_{v \uparrow K}^{\beta},
$$

$$
E_{v}^{\rho}(g)=v(g)^{*} \otimes I E_{v \uparrow K}^{\beta} v(g) \otimes I .
$$

Proof. Let us extend $\beta$ and $\rho$ to unitary representations of $K$ and $G$, respectively, on the $L^{2}$-completions of $\mathcal{F}$ and $\operatorname{Ind}(\mathcal{F})$ for the unique invariant traces. The extension of $\rho$ is clearly the representation induced from the extension of $\beta$ in the sense of Mackey. Extending in this way does not increment the spectra. Hence $L_{v}^{\rho}$ may be determined by the classical Frobenius reciprocity theorem, showing that $T \rightarrow T^{\prime}$ is a linear isomorphism. It is easily checked to be an isometry. Therefore

$$
Z_{v}^{\rho}\left(\bar{T}^{\prime}\right)(g)=\sum_{i} \psi_{i} \otimes T\left(v(g) \psi_{i}\right)^{*},
$$

showing that if $\left(\psi_{i}\right)$ is an orthonormal basis of $H_{v}$ and $\left(\bar{T}_{j}\right)$ an orthonormal basis of $\bar{L}_{v}$, then $\left(\xi_{j}^{\prime}\right)$, where $\xi_{j}^{\prime}(g):=\sum \psi_{k} \otimes T_{j}\left(v(g) \psi_{k}\right)^{*}$, is an orthonormal basis of the Hilbert space of $G$-fixed vectors in $H_{v} \otimes \operatorname{Ind}(\mathcal{F})$, hence the $j r$-entry of $Z_{v}^{\rho}$ is the function

$$
T_{r}\left(v(g) \psi_{j}\right)^{*}=\left(v(g)^{*} \otimes I Z_{v \uparrow K}^{\beta}\right)_{j r} .
$$

If $z$ and $v$ are representations of $K$, we identify the space of bounded adjointable $\mathscr{F}$-module maps $\mathscr{L}_{\mathcal{F}}\left(H_{z} \otimes \mathcal{F}, H_{v} \otimes \mathcal{F}\right)$ with $\mathscr{L}\left(H_{z}, H_{v}\right) \otimes \mathscr{F}$. Hence

$$
(z \otimes \beta, v \otimes \beta)=\left\{T \in \mathscr{L}\left(H_{z}, H_{v}\right) \otimes \mathscr{F} \mid \iota \otimes \beta_{k}(T)=v(k)^{*} \otimes I T z(k) \otimes I\right\} .
$$

As a $K$-space, this space is linearly isomorphic to

$$
\left(H_{v \otimes \bar{z}} \otimes \mathcal{F}\right)^{v \otimes \bar{z} \otimes \beta}=\bar{L}_{v \otimes \bar{z}}^{\beta}
$$

and therefore finite-dimensional. This remark, combined with the previous proposition, shows the following result, needed later. A module map $T \in \mathscr{L}_{\operatorname{Ind}(\mathcal{F})}(H \otimes$ $\left.\operatorname{Ind}(\mathcal{F}), H^{\prime} \otimes \operatorname{Ind}(\mathscr{F})\right)$ will be regarded as a function $T: G \rightarrow \mathscr{L}\left(H, H^{\prime}\right) \otimes \mathscr{F}$. 
Corollary 11.5. There is a full and faithful ${ }^{*}$-functor from the full subcategory of $\operatorname{Mod}_{\rho}(G)$ with objects $v \otimes \rho, v \in \operatorname{Rep}(G)$, to the category $\operatorname{Mod}_{\beta}(K)$, given by

$$
v \otimes \rho \rightarrow v \uparrow_{K} \otimes \beta, \quad T \in\left(v \otimes \rho, v^{\prime} \otimes \rho\right) \rightarrow T(1) \in\left(v \uparrow_{K} \otimes \beta, v^{\prime} \uparrow_{K} \otimes \beta\right) .
$$

The inverse map on arrows is given by $A \rightarrow A^{\prime}$ with $A^{\prime}(g):=v^{\prime}(g)^{*} \otimes I A v(g) \otimes I$.

The functor $T \rightarrow T(1)$ defined in the above corollary will be referred to as the evaluation functor.

Given a projection $E \in \mathscr{L}\left(H_{v}\right) \otimes \mathscr{F}$ and a unital ${ }^{*}$-homomorphism $\eta: \mathcal{F} \rightarrow$ $E \mathscr{L}\left(H_{v}\right) \otimes \mathscr{F} E$ defining a full bimodule structure on the intermediate $K$-module $Y=$ $E H_{v} \otimes \mathcal{F}$, i.e., satisfying conditions (11.1)-(11.4), we may consider the projection $\widetilde{E} \in C\left(G, \mathscr{L}\left(H_{v}\right) \otimes \mathscr{F}\right) \simeq \mathscr{L}\left(H_{v}\right) \otimes C(G, \mathcal{F})$,

$$
\widetilde{E}(g):=v(g)^{*} \otimes I E v(g) \otimes I,
$$

which clearly satisfies

$\iota \otimes \beta_{k}(\widetilde{E}(g))=v(g)^{*} \otimes I \iota \otimes \beta_{k}(E) v(g) \otimes I=v(k g)^{*} \otimes I E v(k g) \otimes I=\widetilde{E}(k g)$,

hence $\widetilde{E} \in \mathscr{L}\left(H_{v}\right) \otimes \operatorname{Ind}(\mathcal{F})$. We may also consider the map taking a continuous function $f$ on $G$ with values in $\mathscr{F}$ to the function

$$
\tilde{\eta}(f)(g):=v(g)^{*} \otimes I \eta(f(g)) v(g) \otimes I .
$$

Similar computations and (11.3) show that if $f \in \operatorname{Ind}(\mathscr{F})$ then $\tilde{\eta}(f) \in \mathscr{L}\left(H_{v}\right) \otimes$ $\operatorname{Ind}(\widetilde{F})$ and $\widetilde{E} \tilde{\eta}(f)=\tilde{\eta}(f)=\tilde{\eta}(f) \widetilde{E}$, hence $\tilde{\eta}$ is in fact a unital ${ }^{*}$-homomorphism between

$$
\tilde{\eta}: \operatorname{Ind}(\mathscr{F}) \rightarrow \widetilde{E} \mathscr{L}\left(H_{v}\right) \otimes \operatorname{Ind}(\widetilde{F}) \widetilde{E},
$$

and $(\widetilde{E}, \tilde{\eta})$ defines a bimodule over the induced algebra $\operatorname{Ind}(\mathscr{F})$. We shall refer to it as the induced bimodule.

Theorem 11.6. The induced bimodule $(\widetilde{E}, \tilde{\eta})$ satisfies (11.1)-(11.4) if $(E, \eta)$ does. Furthermore, if $\mathcal{F}$ is the completion of the dense ${ }^{*}$-subalgebra of $K$-finite elements in the maximal $C^{*}$-norm, any intermediate projective $G$-module $X_{v}^{\rho} \subset Y \subset H_{v} \otimes$ $\operatorname{Ind}(\mathcal{F})$ with a full bimodule structure is defined by such a pair $(E, \eta)$.

Proof. The validity of (11.1)-(11.4) for a bimodule induced from one with analogous properties follows easily from the previous proposition. Conversely, let $\left(E^{\prime}, \eta^{\prime}\right)$ satisfy (11.1)-(11.4) with respect to the automorphism group $\rho$ of the induced algebra. By (11.2), $v(g) \iota \otimes \rho_{g}\left(E^{\prime}\right) v(g)^{*} \otimes I=E^{\prime}$. Evaluating in $g^{\prime}$ gives $v(g) \otimes$ $I E^{\prime}\left(g^{\prime} g\right) v(g)^{*} \otimes I=E^{\prime}\left(g^{\prime}\right)$, hence $E^{\prime}(g)=v(g)^{*} \otimes I E v(g) \otimes I$, where $E:=E^{\prime}(1)$. It is now clear that $E$ satisfies (11.1). Moreover, for $k \in K$,

$$
v(k)^{*} \otimes I E v(k) \otimes I=E^{\prime}(k)=\iota \otimes \beta_{k}\left(E^{\prime}(1)\right),
$$

hence $E$ satisfies (11.2). 
On the other hand, $E^{\prime} \mathscr{L}\left(H_{v}\right) \otimes \operatorname{Ind}(\mathcal{F}) E^{\prime}$, with $G$-action $\operatorname{Ad} v \otimes \rho$, is isomorphic to the $\mathrm{C}^{*}$-system induced by $E \mathscr{L}\left(H_{v}\right) \otimes \mathscr{F} E$ with $K$-action $\operatorname{Ad} v \uparrow_{K} \otimes \beta$. An explicit $G$-equivariant isomorphism takes $f \in E^{\prime} \mathscr{L}\left(H_{v}\right) \otimes \operatorname{Ind}(\mathcal{F}) E^{\prime}$ to the element of $C\left(G, E \mathscr{L}\left(H_{v}\right) \otimes \mathscr{F} E\right)$ defined by $g \in G \rightarrow \operatorname{Ad} v(g) \otimes I f(g)$. Therefore condition (11.3) can be regarded as an intertwining relation between induced group representations. Hence, by Frobenius reciprocity, there is a map, a priori just linear, and densely defined on the ${ }^{*}$-subalgebra of $K$-finite elements, $\eta: \mathscr{F} \rightarrow E \mathscr{L}\left(H_{v}\right) \otimes$ $\mathcal{F} E$ satisfying the intertwining relation

$$
\eta\left(\beta_{k}(f)\right)=\operatorname{Ad} v_{k} \otimes \beta_{k}(\zeta(f)),
$$

and hence (11.3), for $f \in \mathcal{F}, k \in K$, inducing $\eta^{\prime}$ via

$$
\eta^{\prime}(f)(g)=\operatorname{Ad} v(g)^{*} \otimes I \eta(f(g)) .
$$

We show that $\eta$ is a unital ${ }^{*}$-homomorphism. It is well known that for any $K$-finite element $f_{1} \in \mathcal{F}$, there is an element $f \in \operatorname{Ind}(\mathscr{F})$ with $f_{1}=f(1)$. Thus, (11.4) follows. On the other hand, since $\eta^{\prime}$ is a unital *-homomorphism, the above formula, evaluated in 1 , shows that $\eta$ is a unital ${ }^{*}$-homomorphism on the dense ${ }^{*}$-subalgebra of $K$-finite elements. Since $\mathscr{F}$ is the completion in the maximal $\mathrm{C}^{*}$-norm, we may conclude that the unique extension of $\eta$ to $\mathscr{F}$ has the required properties.

11.4. Classification of intermediate full bimodule representations for type I ergodic actions. Since a type I ergodic action of a compact group $G$ is induced by an ergodic action $\beta$ of a closed subgroup $K$ on a matrix algebra and since all intermediate full submodule representations for the ergodic action of $G$ are induced by similar submodules for the action of $K$ (Theorem 11.6), it suffices to classify the intermediate full submodule representations for the action of the subgroup.

Let the compact group $K$ act on $\mathcal{F}$. We first give a simple method of constructing extensions of $X_{v}^{\beta}$ to full bimodule representations on projective submodules of $H_{v} \otimes \mathcal{F}$.

Proposition 11.7. Pick a representation $v$ of $K$.

(a) If there is a unitary representation $z$ of $K$ with

$$
\operatorname{dim}(z) \leq \operatorname{dim}(v)-\operatorname{dim}\left(\bar{L}_{v}\right)
$$

and an isometry

$$
W \in(z \otimes \beta, v \otimes \beta) \text { such that } W^{*} Z_{v}=0,
$$

then the intermediate $K$-module subrepresentation $X_{v} \subset Y \subset H_{v} \otimes \mathscr{F}$ defined by the projection $E:=Z_{v} Z_{v}^{*}+W W^{*} \in \mathscr{L}\left(H_{v}\right) \otimes \mathcal{F}$ becomes a full bimodule $K$-representation with left action $\eta(f):=Z_{v} I \otimes f Z_{v}^{*}+W I \otimes f W^{*}$.

(b) If we can choose $z$ with $\operatorname{dim}(z)=\operatorname{dim}(v)-\operatorname{dim}\left(\bar{L}_{v}\right)$, then we get a full $K$-bimodule representation for $v \otimes \beta$. 
Proof. $E$ and $\eta$ defined as in (a) certainly satisfy the assumptions of Proposition 11.2, hence we get a full bimodule representation $Y$, and (b) clearly follows.

We next provide a complete list if $\mathscr{F}$ is a matrix algebra.

Theorem 11.8. Let $\beta$ be an ergodic action of a compact group $K$ on a factor $\mathcal{F}$ and $v$ a representation of $K$.

(a) Two pairs $(z, W),\left(z^{\prime}, W^{\prime}\right)$ satisfying (11.5) and (11.6) define the same intermediate $K$-bimodule representation $Y$ if and only if there is a unitary intertwiner $U \in\left(z, z^{\prime}\right)$ such that $W=W^{\prime} U \otimes I$.

(b) If $\mathcal{F}$ is a matrix algebra, then any full intermediate bimodule representation $X_{v} \subset Y \subset H_{v} \otimes \mathcal{F}$ arises from a pair $(z, W)$. In particular, full $K$-bimodule representations on $H_{v} \otimes \mathscr{F}$ correspond to pairs $(z, W)$ satisfying (11.5) and (11.6), where the inequality of (11.5) is strengthened to an equality.

Proof. (a) Obviously two equivalent pairs $(z, W),\left(z^{\prime}, W^{\prime}\right)$, as in a), give rise to the same intermediate $K$-module $Y$ with the same left action $\eta$. Conversely, suppose $(z, W)$ and $\left(z^{\prime}, W^{\prime}\right)$ define the same $K$-bimodule representation $Y$. Then clearly $W W^{*}=W^{\prime} W^{\prime *}$. Since the two left actions coincide, $W I \otimes f W^{*}=W^{\prime} I \otimes f W^{\prime *}$. Hence the unitary $W^{\prime *} W \in\left(z \otimes \beta, z^{\prime} \otimes \beta\right)$ is of the form $U \otimes I$, with $U: H_{z} \rightarrow H_{z^{\prime}}$, as $\mathscr{F}$ is a factor. Thus $W=W^{\prime} U \otimes I$. Making the intertwining property of $W$ and $W^{\prime}$ explicit shows that $U \in\left(z, z^{\prime}\right)$.

(b) Assume that $\mathscr{F}=\operatorname{Mat}_{r}(\mathbb{C})$. If $Y$ is defined by $E$ and $\eta$, then $E$ needs to be of rank $q r$ with $q$ integer, as $\eta$ is unital, and $q \geq \operatorname{dim}\left(\bar{L}_{v}\right)$ as $E \geq E_{v}$. Set $\eta_{1}(f):=\eta(f)\left(E-E_{v}\right)$. We can write $\eta_{1}$ in the form $\eta_{1}(f)=W I \otimes f W^{*}$ with $W$ a partial isometry such that $W W^{*}=E-E_{v}=: E_{1}$ and $W^{*} W \in \mathscr{L}\left(H_{v}\right) \otimes \mathbb{C}$. The relation $W^{*} Z_{v}=0$ implies $\operatorname{dim}\left(W^{*} W H_{v}\right)+\operatorname{dim}\left(\bar{L}_{v}\right) \leq \operatorname{dim}(v)$. The covariance condition (3.5) for $Y$ becomes

$$
\operatorname{Ad} v(k) \otimes \beta_{k} \eta(f)=\eta\left(\beta_{k}(f)\right), \quad f \in \operatorname{Mat}_{r}(\mathbb{C}),
$$

and is equivalent to requiring an analogous relation for $\eta_{1}$ :

$$
v(k) \otimes I \iota \otimes \beta_{k}(W) I \otimes \beta_{k}(f) \iota \otimes \beta_{k}\left(W^{*}\right) v(k)^{*} \otimes I=W I \otimes \beta_{k}(f) W^{*},
$$

or

$$
W^{*} v(k) \otimes I \iota \otimes \beta_{k}(W) \in \mathscr{L}\left(H_{v}\right) \otimes \mathbb{C} .
$$

On the other hand, the map $k \rightarrow z(k)$ with $z(k)$ defined by

$$
z(k) \otimes I:=W^{*} v(k) \otimes I \iota \otimes \beta_{k}(W)
$$

is a unitary representation of $K$ on the subspace $W^{*} W H_{v}$, completing the proof of (b). 
The following result summarizes the classification of full bimodule representations for type I ergodic actions achieved here.

Theorem 11.9. Let $\mathcal{F}$ be a matrix algebra, and let $\beta$ be an ergodic action of a closed subgroup $K$ of a compact group $G$ on $\mathcal{F}$. Pick a unitary finite-dimensional representation $v$ of $G$. Then:

(a) The full bimodule $G$-representations over intermediate projective $G$-module subrepresentations $X_{v} \subset Y \subset H_{v} \otimes \operatorname{Ind}(\mathcal{F})$ are classified by equivalence classes of pairs $(z, W)$, where $z$ is a unitary finite-dimensional representation of $K$ and $W \in\left(z \otimes \beta, v \uparrow_{K} \otimes \beta\right)$ an isometry satisfying

$$
W^{*} Z_{v \uparrow K}^{\beta}=0 .
$$

$(W, z)$ and $\left(W^{\prime}, z^{\prime}\right)$ are equivalent if there is a unitary intertwiner $U \in\left(z, z^{\prime}\right)$ with $W=W^{\prime} U \otimes I$.

(b) In particular, the full $G$-bimodule representations on $H_{v} \otimes \operatorname{Ind}(\mathcal{F})$ correspond to pairs $(W, z)$ where $W$ satisfies

$$
W W^{*}+Z_{v \uparrow K}^{\beta} Z_{v \uparrow K}^{\beta *}=I .
$$

The corresponding left module structure $\tilde{\eta}: \operatorname{Ind}(\mathscr{F}) \rightarrow \mathscr{L}\left(H_{v}\right) \otimes \operatorname{Ind}(\mathcal{F})$ is given by

$$
\tilde{\eta}(f)(g)=v(g)^{*} \otimes I\left(Z_{v{ }_{\uparrow K}}^{\beta} I \otimes f(g) Z_{v \uparrow_{K}}^{\beta *}+W I \otimes f(g) W^{*}\right) v(g) \otimes I .
$$

Remark. If the module $G$-representation $v \otimes \rho$ over $\operatorname{Ind}(\mathscr{F})$ can be made into a full bimodule $G$-representation, and if it is induced by the pair $(z, W)$, we may form the $K$-representation $z^{\prime}:=\iota_{L_{v}} \oplus z$ of the same dimension as $v$. Then $Z_{v{ }_{1}}^{\beta} \oplus W$ is a unitary equivalence from the original full bimodule structure for $v \uparrow_{K} \otimes \beta$ inducing the given full bimodule structure for $v \otimes \rho$, in the sense of Theorem 11.6, to $z^{\prime} \otimes \beta$ with the trivial left module structure. This remark will play a role in the proof of Theorem 6.7.

As a consequence of (b) of Theorem 11.8, the module representation $v \otimes \beta$, with $v$ in the spectrum, in some cases, does not admit any full bimodule $K$-representation unless $v$ has full multiplicity. We discuss a class of examples.

Example 11.10. Consider the adjoint action $\beta_{r}$ of the unique $r+1$-dimensional irreducible representation $v_{r}$ of the group $K=\mathrm{SU}(2)$ acting on the matrix algebra $\operatorname{Mat}_{r+1}(\mathbb{C})$. We show that if $r \geq 1, v \otimes \beta_{r}$ becomes a full bimodule representation only for certain $v$. Hence none of the actions $\beta_{r}$ arise from a relaxed tensor functor $\operatorname{Rep}(\mathrm{SU}(2)) \rightarrow \mathcal{M}$ to a tensor $\mathrm{C}^{*}$-category, as this functor would make all $v \otimes \beta_{r}$ into full bimodule representations by Theorem 6.2. 
The spectrum of $\beta_{r}$ may be determined by the Clebsch-Gordan rule

$$
v_{r} \otimes v_{s} \simeq v_{r-s} \oplus v_{r-s+2} \oplus \cdots \oplus v_{r+s}, \quad r \geq s,
$$

after regarding $\beta_{r}$ as a Hilbert space representation with respect to the inner product defined by the ( $K$-invariant) trace of $\operatorname{Mat}_{r+1}(\mathbb{C}) . v_{r}$ being selfconjugate, we have $\beta_{r} \simeq v_{r} \otimes v_{r} \simeq v_{0} \oplus v_{2} \oplus \cdots \oplus v_{2 r}$. Hence any spectral representation has multiplicity 1 .

In particular, $v_{1}$ is never in the spectrum of $\beta_{r}$, and the full bimodule structures on $H_{v_{1}} \otimes \operatorname{Mat}_{r+1}(\mathbb{C})$ are described by pairs $(z, W)$ with $\operatorname{dim}(z)=\operatorname{dim}\left(v_{1}\right)=2$. Since $z$ can never contain the trivial representation, we necessarily have $z=v_{1}$. Hence we need to specify a unitary

$$
\begin{gathered}
W \in\left(v_{1} \otimes \beta_{r}, v_{1} \otimes \beta_{r}\right) \simeq \\
\left(v_{1} \otimes v_{r}, v_{1} \otimes v_{r}\right) \simeq\left(v_{r-1} \oplus v_{r+1}, v_{r-1} \oplus v_{r+1}\right) \simeq \mathbb{C} \oplus \mathbb{C} .
\end{gathered}
$$

Hence $v_{1} \otimes \beta_{r}$ admits full bimodule structures, and they are classified by $\mathbb{T}$.

On the other hand, low multiplicity of a representation in the spectrum in general rules out full bimodule structures on $v \otimes \beta_{r}$ as the following simple argument shows. If there were a structure of a full $K$-bimodule representation on $v_{2} \otimes \beta_{r}$ defined by $(z, W)$, then we must have $\operatorname{dim}(z)=\operatorname{dim}\left(v_{2}\right)-\operatorname{mult}\left(v_{2}\right)=2$. Since $z$ cannot contain the trivial representation, $z=v_{1}$. On the other hand the space of module intertwiners $\left(v_{1} \otimes \beta_{r}, v_{2} \otimes \beta_{r}\right)$ is isomorphic to $\left(v_{1} \otimes v_{r}, v_{2} \otimes v_{r}\right)$ which is trivial, again by the Clebsch-Gordan rule. Hence $v_{2} \otimes \beta_{r}$ admits no full bimodule structure, and actually $X_{v_{2}}$ admits no proper extension to a full bimodule representation.

Corollary 11.11. Let $K$ act ergodically on a matrix algebra.

(a) Let $v$ be a representation with $\bar{L}_{v} \neq\{0\}$ and assume that any irreducible of smaller dimension has full multiplicity. If $\operatorname{dim}\left(\bar{L}_{v}\right)<\operatorname{dim}(v)$, then $X_{v}$ does not admit any proper extension to a full bimodule $K$-representation.

(b) If $\beta$ has full spectrum (hence $K$ is finite) and each $v \otimes \beta$ can be made into a full bimodule representation, then each irreducible is of full multiplicity in $\beta$.

Proof. Let $z$ and $W$ be as required in (a) of Proposition 11.7. Since $\operatorname{dim}(z)<\operatorname{dim}(v)$, any irreducible subrepresentation of $z$ has full multiplicity in $\beta$. Hence there is a unitary $U \in \mathscr{L}\left(H_{z}\right) \otimes \operatorname{Mat}_{r}(\mathbb{C})$ with $\iota \otimes \beta_{k}(U)=z(k)^{*} \otimes I U$. Hence every column of $W U$ gives an element of $\bar{L}_{v}$ orthogonal to $\bar{L}_{v}$ itself, as $W^{*} Z_{v}=0$. So $W U=0$ and $W=0$. This completes the proof of (a), and (b) follows easily.

\section{Tensorial properties of the evaluation functor}

In this section we use the classification of full Hilbert bimodule structures on type I von Neumann algebras obtained in the previous section to prove Theorem 6.7 , and Corollaries 6.8 and 6.9. 
We need a few simple lemmas that clarify the tensorial properties of the evaluation functor defined in the previous section. We thus assume that we are given an action $\beta$ of a closed subgroup $K$ of a compact group $G$ on a $C^{*}$-algebra $\mathcal{F}$ and that for each $v \in \operatorname{Rep}(G)$ we have a full bimodule structure for $v \uparrow_{K} \otimes \beta$ defined by the ${ }^{*}$-homomorphism $\eta_{v}: \mathcal{F} \rightarrow \mathscr{L}\left(H_{v}\right) \otimes \mathscr{F}$. We consider the full bimodule structure $\tilde{\eta}_{v}$ for $v \otimes \rho$ induced by $\eta_{v}$ as in Section 11.3.

Lemma 12.1. If $T \in\left(v \otimes \rho, v^{\prime} \otimes \rho\right)$ is a bimodule map, then $T(1) \in\left(v \uparrow_{K} \otimes\right.$ $\left.\beta, v^{\prime} \uparrow_{K} \otimes \beta\right)$ is a bimodule map as well.

Proof. The proof is straightforward. By Corollary 11.5, we may write $T$ in the form $T(g)=v^{\prime}(g)^{*} \otimes I T(1) v(g) \otimes I$, with $T(1) \in\left(v \uparrow_{K} \otimes \beta, v^{\prime} \uparrow_{K} \otimes \beta\right)$. The intertwining relation for $T$ evaluated at 1 gives the intertwining relation for $T(1)$.

Let us now consider a unital $\mathrm{C}^{*}$-algebra $\ell$ and two finite-dimensional Hilbert spaces $H$ and $L$. Consider the right $\mathrm{C}^{*}$-modules $H \otimes \ell$ and $L \otimes \ell$. If $L \otimes \ell$ also has a left $\ell$-module structure defined by a unital ${ }^{*}$-homomorphism $\eta: \ell \rightarrow \mathscr{L}_{\mathcal{C}}(L) \otimes \mathcal{C}$ then we may form the tensor product right Hilbert $C^{*}$-module $(H \otimes \mathcal{C}) \otimes \mathcal{C}(L \otimes \mathcal{C})$, to be identified with $(H \otimes L) \otimes \mathcal{C}$. We may thus form tensor products $T \otimes S$ of a module intertwiner $T \in \mathscr{L}\left(H, H^{\prime}\right) \otimes \mathcal{C}$ with a bimodule intertwiner $S \in \mathcal{C} \mathscr{L}\left(L \otimes \mathcal{C}, L^{\prime} \otimes \mathcal{C}\right)$ giving an element of $\mathscr{L}\left(H \otimes L, H^{\prime} \otimes L^{\prime}\right) \otimes \mathcal{C}$.

Lemma 12.2. Let us consider $H_{v} \otimes \operatorname{Ind}(\widetilde{F})$ and $H_{v^{\prime}} \otimes \operatorname{Ind}(\mathcal{F})$ as right $\operatorname{Ind}(\widetilde{F})$ modules. Let $\tilde{\eta}_{u}, \tilde{\eta}_{u^{\prime}}$ make $H_{u} \otimes \operatorname{Ind}(\mathcal{F})$ and $H_{u^{\prime}} \otimes \operatorname{Ind}(\tilde{F})$ into $\operatorname{Ind}(\mathcal{F})$-bimodules. For a module intertwiner $T \in\left(v \otimes \rho, v^{\prime} \otimes \rho\right)$ and a bimodule intertwiner $S \in$ $\left(u \otimes \rho, u^{\prime} \otimes \rho\right)$, we have

$$
(T \otimes S)(1)=T(1) \otimes S(1) .
$$

Proof. Notice that $S(1)$ is a bimodule intertwiner by the previous lemma, hence the right-hand side makes sense. Let $H, H^{\prime}, L, L^{\prime}$ be finite-dimensional Hilbert spaces and $\eta, \eta^{\prime}$ left $\zeta$-module structures on $L \otimes \mathcal{C}$ and $L^{\prime} \otimes \mathcal{C}$ respectively. Given a module intertwiner $T \in \mathscr{L}\left(H, H^{\prime}\right) \otimes \mathcal{C}$, and a bimodule intertwiner $S \in \mathcal{C}^{\mathscr{L}} \mathcal{C}\left(L \otimes \mathcal{C}, L^{\prime} \otimes \mathcal{C}\right)$, a simple computation shows that if $T$ is represented by the $\mathcal{C}$-valued matrix $\left(t_{r s}\right)$, in the sense that $T=\sum_{r s} e_{r s} \otimes t_{r s}$, where $e_{r s}$ are matrix units, and if $S$ is represented by $\left(s_{p q}\right)$ then the module intertwiner $T \otimes S$ regarded as an element of $\mathscr{L}\left(H \otimes L, H^{\prime} \otimes\right.$ $\left.L^{\prime}\right) \otimes C$ is represented by the matrix whose $(r p)(s q)$-entry is $\sum_{h} \eta^{\prime}\left(t_{r s}\right)_{p h} s_{h q}$. We apply this to $H_{v}, H_{v^{\prime}}, H_{u}, H_{u^{\prime}}$ and $\operatorname{Ind}(\mathcal{F})$ respectively. By Corollary 11.5 , we may write $\left(t_{r s}\right)(g)=v^{\prime}(g)^{*} T(1) v(g),\left(s_{p q}\right)(g)=u^{\prime}(g)^{*} S(1) u(g)$, where $T(1)$ and $S(1)$ are now represented by $\mathcal{F}$-valued matrices. Recalling how $\tilde{\eta}_{u^{\prime}}$ was defined before Theorem 11.6, the $(r p)(s q)$-entry of $T \otimes S$ is the function

$$
\begin{aligned}
& \sum_{h} \tilde{\eta}_{u^{\prime}}\left(t_{r s}\right)_{p h}(g) s_{h q}(g) \\
& \quad=\sum_{h, l, m}{\overline{u^{\prime}(g)}}_{l p} \eta_{u^{\prime}}\left(t_{r s}(g)\right)_{l m} u^{\prime}(g)_{m h} s_{h q}(g)
\end{aligned}
$$




$$
\begin{aligned}
& =\sum_{h, l, m, i, j, k, t}{\overline{u^{\prime}(g)}}_{l p}{\overline{v^{\prime}(g)}}_{i r} \eta_{u^{\prime}}\left(T(1)_{i j}\right)_{l m} v(g)_{j s} u^{\prime}(g)_{m h}{\overline{u^{\prime}(g)}}_{k h} S(1)_{k t} u(g)_{t q} \\
& =\sum_{l, i, j, k, t}{\overline{u^{\prime}(g)}}_{l p}{\overline{v^{\prime}(g)}}_{i r} \eta_{u^{\prime}}\left(T(1)_{i j}\right)_{l k} v(g)_{j s} S(1)_{k t} u(g)_{t q} \\
& =\sum_{l, i, j, k, t} v^{\prime} \otimes u^{\prime}(g)_{(r p)(i l)}^{*} \eta_{u^{\prime}}\left(T(1)_{i j}\right)_{l k} S(1)_{k t} v \otimes u(g)_{(j t)(s q)} \\
& =\sum_{l, i, j, k, t} v^{\prime} \otimes u^{\prime}(g)_{(r p)(i l)}^{*}(T(1) \otimes S(1))_{(i l)(j t)} v \otimes u(g)_{(j t)(s q)} .
\end{aligned}
$$

Hence $(T \otimes S)(1)=T(1) \otimes S(1)$.

Note that if $H \otimes \mathcal{C}$ and $L \otimes \mathcal{C}$ have left bimodule structures defined by $\eta: \mathcal{C} \rightarrow$ $\mathscr{L}(H) \otimes \mathscr{C}$ and $\zeta: \mathcal{C} \rightarrow \mathscr{L}(L) \otimes \mathscr{C}$ then under the unitary module map $(H \otimes \mathscr{C}) \otimes \mathcal{C}$ $(L \otimes \mathcal{C}) \simeq(H \otimes L) \otimes \mathcal{C}$ the left module structure $\mathcal{C} \rightarrow \mathscr{L}(H \otimes L) \otimes \mathcal{C}$ corresponding to the tensor product bimodule is given by $\iota_{\mathscr{L}(H)} \otimes \zeta \circ \eta, \iota \mathscr{L}(H)$ being the identity map on $\mathscr{L}(H)$. This tensor product left action will be denoted by $\eta \otimes \zeta$.

Lemma 12.3. If the induced set of left actions $\left\{\tilde{\eta}_{u}, u \in \operatorname{Rep}(G)\right\}$ on the $C^{*}$-modules $H_{u} \otimes \operatorname{Ind}(\mathcal{F})$ is tensorial, i.e., $\tilde{\eta}_{u \otimes v}=\tilde{\eta}_{u} \otimes \tilde{\eta}_{v}$ for $u, v \in \operatorname{Rep}(G)$, then the original set $\left\{\eta_{u}, u \in \operatorname{Rep}(G)\right\}$ is tensorial too.

Proof. It suffices to evaluate the tensorial relation for the $\tilde{\eta}_{u}$ 's at 1 .

We summarize the above lemmas as follows.

Theorem 12.4. Let $\beta$ be an action of a closed subgroup $K$ of a compact group $G$ on $a C^{*}$-algebra $\mathcal{F}$. Assume that for each $v \in \operatorname{Rep}(G)$ we have a full bimodule structure for $v \uparrow_{K} \otimes \beta$ defined by the ${ }^{*}$-homomorphism $\eta_{v}: \mathcal{F} \rightarrow \mathscr{L}\left(H_{v}\right) \otimes \mathcal{F}$. If the set of induced bimodule structures $\tilde{\eta}_{v}$ for $v \otimes \rho$ is tensorial, then the evaluation functor $T \rightarrow T(1)$ restricts to a faithful tensor functor from the full tensor $C^{*}$-subcategory of $\operatorname{Bimod}_{\rho}(G)$ with objects $v \otimes \rho$ to $\operatorname{Bimod}_{\beta}(K)$.

Proof of Theorem 6.7 and Corollary 6.8. Theorem 6.2, applied to the given tensor functor $\mu: \delta_{G} \rightarrow \mathcal{M}$ and to the embedding functor $\tau: \delta_{G} \rightarrow$ Hilb, allows us to identify $\mathcal{M}_{\mu}^{\otimes}$ with the full subcategory of $\operatorname{Bimod}_{\alpha}(G)$ with objects $u^{r} \otimes \alpha, r=$ $0,1,2, \ldots$, where $u$ is the distinguished representation of $G$ and, as before, $\alpha$ is the ergodic action of $G$ on the associated $\mathrm{C}^{*}$-algebra $\mathcal{C}$. That theorem provides us with a full $G$-bimodule representation for each $u^{r} \otimes \alpha$ and the collection of these left module structures is tensorial. Since the von Neumann completion of $\mathcal{C}$ in the GNS representation of the $G$-invariant trace state is of type I, we may identify the completed ergodic system with a von Neumann ergodic system $(\operatorname{Ind}(\mathcal{F}), \rho)$ induced from a closed subgroup $K$, unique up to conjugation, where $\mathcal{F}$ is a matrix algebra with an ergodic action $\beta$ of $K$. The left $\ell$-action on $H_{u^{r}} \otimes \mathcal{C}$ is defined by a unital ${ }^{*}$-homomorphism $\eta: \mathscr{C} \rightarrow \mathscr{L}\left(H_{u}\right) \otimes \mathscr{C}$ intertwining $\alpha$ with $\operatorname{Ad}(u) \otimes \alpha$ by 
Proposition 11.2. Hence, if tr and $\tau$ are the normalized $G$-invariant traces on $\mathscr{L}\left(H_{u}\right)$ and $\mathcal{C}$ respectively, $(\operatorname{tr} \otimes \tau) \circ \eta$ is a $G$-invariant trace on $\mathcal{C}$. Such a trace is unique so $(\operatorname{tr} \otimes \tau) \circ \eta=\tau$. Thus $\eta$ induces a normal ${ }^{*}$-homomorphism from $\operatorname{Ind}(\mathcal{F})$ to $\mathscr{L}\left(H_{u}\right) \otimes$ $\operatorname{Ind}(\mathcal{F})$. Correspondingly, we get a set of tensorial full bimodule structures for $u^{r} \otimes \rho$. Thus by Theorem 12.4 there is a faithful tensor functor from the full subcategory of $\operatorname{Bimod}_{\rho}(G)$ with objects $u^{r} \otimes \rho$ to the full subcategory $\mathcal{T}$ of $\operatorname{Bimod}_{\beta}(K)$ with objects $u^{r} \uparrow_{K} \otimes \beta$. We next apply Theorem 11.9 to the full bimodule $K$-representation $u \uparrow_{K} \otimes \beta$ fixing a pair $(z, W)$. We set $z^{\prime}:=\iota_{\bar{L}_{u \uparrow_{K}}^{\beta}} \oplus z$ and $U:=Z_{u \uparrow_{K}} \oplus W$, a $K$-bimodule unitary in $\left(z^{\prime} \otimes \beta, u \uparrow_{K} \otimes \beta\right)$ if $z^{\prime}$ has the trivial left $\mathcal{C}_{\text {-action. We define }}$ $\mathrm{a}^{*}$-functor $\mathcal{T} \rightarrow \operatorname{Rep}(K)$ taking $u^{r} \uparrow_{K} \otimes \beta$ to $z^{\prime r}$ and a bimodule intertwiner $T \in$ $\left(u^{r} \uparrow_{K} \otimes \beta, u^{s} \uparrow_{K} \otimes \beta\right)$ to $U^{* \otimes s} T U^{\otimes r}$, which is tensorial to the category of Hilbert bimodule representations. We need to show that any arrow is in fact an arrow in the category $\operatorname{Rep}(K)$ regarded as embedded into the category of bimodule representations as a tensor $\mathrm{C}^{*}$-category. In other words, we need to show that $U^{* \otimes s} T U^{\otimes r}$ lies in the subspace $\mathscr{L}\left(H_{z^{\prime}} r, H_{z^{\prime}} s\right) \otimes \mathbb{C}$ of $\mathscr{L}\left(H_{z^{\prime}}, H_{z^{\prime}} s\right) \otimes \mathcal{F}$. To this end, recall that Theorem 6.2 ensures that any module $G$-intertwiner is in fact a bimodule intertwiner, see Theorem 9.6. The same property holds for the bimodule structures of the $u^{r} \uparrow_{K} \otimes$ $\beta$ 's and hence for the bimodule structures of the $z^{\prime r} \otimes \beta$, unitarily related to them, since the evaluation functor is full and faithful, see Corollary 11.5. But now each $z^{\prime r} \otimes \beta$ has the trivial left module structure over $\mathcal{F}$, hence a bimodule intertwiner lies in $\mathscr{L}\left(H_{z^{\prime}}, H_{z^{\prime}}\right) \otimes Z(\mathcal{F})=\mathscr{L}\left(H_{z^{\prime \prime}}, H_{z^{\prime}}\right) \otimes \mathbb{C}$ since $\mathscr{F}$ is a factor, see the discussion following Proposition 5.1. This argument completes the proof of Theorem 6.7. If in particular $\mathcal{C}$ is commutative then $\mathscr{F}=\mathbb{C}$, and $z^{\prime}=u \uparrow_{K}$, completing the proof of Corollary 6.8 .

Proof of Corollary 6.9. The condition on $R$ allows us to define a tensor functor from $\oint_{\mathrm{SU}(2)}$ to $\mathcal{M}$ taking the defining representation $u$ to $x$ and the determinant element to $R$, see [10]. We may now apply Theorem 6.7.

\section{Appendix}

In this appendix we collect some computations with quasi-tensor functors that we have used throughout the paper.

Proposition 13.1. If we take $1_{\mu_{\bar{v}}} \otimes \widehat{R}_{u} \otimes 1_{\mu_{v}} \circ \widehat{R}_{v}$ as a solution of the conjugate equations for $\mu_{u} \otimes \mu_{v}$ and $\hat{R}_{u \otimes v}$ as the solution for $\mu_{u \otimes v}$, where $\widehat{R}_{u \otimes v}$ is the image solution of the tensor product solution for $u \otimes v$, then

$$
\tilde{\mu}_{u, v}^{\cdot}=\tilde{\mu}_{\bar{v}, \bar{u}}, \quad \tilde{\mu}_{u, v}^{* \bullet}=\tilde{\mu}_{\bar{v}, \bar{u}}^{*} .
$$


Proof. We have

$$
\begin{aligned}
& \tilde{\mu}_{u, v}^{\bullet}=\left(\hat{R}_{v}^{*} \circ 1_{\mu_{\bar{v}}} \otimes \hat{R}_{u}^{*} \otimes 1_{\mu_{v}}\right) \otimes 1_{\mu_{\bar{v} \otimes \bar{u}}} \circ 1_{\mu_{\bar{v}}} \\
& \otimes 1_{\mu_{\bar{u}}} \otimes \tilde{\mu}_{u, v}^{*} \otimes 1_{\mu_{\bar{v} \otimes \bar{u}}} \circ 1_{\mu_{\bar{v}}} \otimes 1_{\mu_{\bar{u}}} \otimes \hat{\bar{R}}_{u \otimes v} \\
& =\left(\hat{R}_{v}^{*} \circ 1_{\mu_{\bar{v}}} \otimes \hat{R}_{u}^{*} \otimes 1_{\mu_{v}}\right) \otimes 1_{\mu_{\bar{v} \otimes \bar{u}}} \circ 1_{\mu_{\bar{v}}} \\
& \otimes 1_{\mu_{\bar{u}}} \otimes\left(\tilde{\mu}_{u, v}^{*} \otimes 1_{\mu_{\bar{v} \otimes \bar{u}}} \circ \tilde{\mu}_{u \otimes v, \bar{v} \otimes \bar{u}}^{*} \circ \mu\left(\bar{R}_{u \otimes v}\right)\right. \\
& =\left(\widehat{R}_{v}^{*} \circ 1_{\mu_{\bar{v}}} \otimes \hat{R}_{u}^{*} \otimes 1_{\mu_{v}}\right) \otimes 1_{\mu_{\bar{v} \otimes \bar{u}}} \circ 1_{\mu_{\bar{v}}} \\
& \otimes 1_{\mu_{\bar{u}}} \otimes\left(1_{\mu_{u}} \otimes \tilde{\mu}_{v, \bar{v} \otimes \bar{u}}^{*} \circ \tilde{\mu}_{u, v \otimes \bar{v} \otimes \bar{u}^{*}}^{*} \mu\left(\bar{R}_{u \otimes v}\right)\right) \\
& =\left(\hat{R}_{v}^{*} \circ 1_{\mu_{\bar{v}}} \otimes \hat{R}_{u}^{*} \otimes 1_{\mu_{v}}\right) \otimes 1_{\mu_{\bar{v} \otimes \bar{u}}} \circ 1_{\mu_{\bar{v}}} \otimes 1_{\mu_{\bar{u}}} \\
& \otimes\left(1_{\mu_{u}} \otimes \tilde{\mu}_{v, \bar{v} \otimes \bar{u}}^{*} \circ 1_{\mu_{u}} \otimes \mu\left(\bar{R}_{v} \otimes 1_{\bar{u}}\right) \circ \tilde{\mu}_{u, \bar{u}}^{*} \circ \mu\left(\bar{R}_{u}\right)\right) \\
& =\left(\hat{R}_{v}^{*} \circ 1_{\mu_{\bar{v}}} \otimes \mu\left(R_{u}^{*}\right) \otimes 1_{\mu_{v}}\right) \otimes 1_{\mu_{\bar{v} \otimes \bar{u}}} \circ 1_{\mu_{\bar{v}}} \otimes 1_{\mu_{\bar{u} \otimes u}} \\
& \otimes\left(\tilde{\mu}_{v, \bar{v} \otimes \bar{u}}^{*} \circ \mu\left(\bar{R}_{v} \otimes 1_{\bar{u}}\right)\right) \circ 1_{\mu_{\bar{v}}} \otimes \tilde{\mu}_{\bar{u}, u} \otimes 1_{\mu_{\bar{u}}} \circ 1_{\mu_{\bar{v}}} \otimes 1_{\mu_{\bar{u}}} \otimes \widehat{R}_{u} \\
& =\widehat{R}_{v}^{*} \otimes 1_{\mu_{\bar{v} \otimes \bar{u}}} \circ 1_{\mu_{\bar{v}}} \otimes \tilde{\mu}_{v, \bar{v} \otimes \bar{u}}^{*} \circ 1_{\mu_{\bar{v}}} \otimes \mu\left(\bar{R}_{v} \otimes 1_{\bar{u}}\right) \circ 1_{\mu_{\bar{v}}} \\
& \otimes \mu\left(R_{u}^{*}\right) \otimes 1_{\mu_{\bar{u}}} \circ 1_{\mu_{\bar{v}}} \otimes \tilde{\mu}_{\bar{u}, u}^{*} \otimes 1_{\mu_{\bar{u}}} \circ 1_{\mu_{\bar{v}}} \otimes 1_{\mu_{\bar{u}}} \otimes \hat{\bar{R}}_{u} .
\end{aligned}
$$

Now

$$
\begin{aligned}
\mu\left(R_{u}^{*}\right) & \otimes 1_{\mu_{\bar{u}}} \circ \tilde{\mu}_{\bar{u}, u} \otimes 1_{\mu_{\bar{u}}} \circ 1_{\mu_{\bar{u}}} \otimes \hat{\bar{R}}_{u} \\
& =\mu\left(R_{u}^{*}\right) \otimes 1_{\mu_{\bar{u}}} \circ \tilde{\mu}_{\bar{u}, u} \otimes 1_{\mu_{\bar{u}}} \circ 1_{\mu_{\bar{u}}} \otimes \tilde{\mu}_{u, \bar{u}}^{*} \circ 1_{\mu_{\bar{u}}} \otimes \mu\left(\bar{R}_{u}\right) \\
& =\mu\left(R_{u}^{*}\right) \otimes 1_{\mu_{\bar{u}}} \circ \tilde{\mu}_{\bar{u} \otimes u, \bar{u}}^{*} \circ \tilde{\mu}_{\bar{u}, u \otimes u \otimes \bar{u}} \circ 1_{\mu_{\bar{u}}} \otimes \mu\left(\bar{R}_{u}\right) \\
& =\mu\left(R_{u}^{*} \otimes 1_{\bar{u}} \circ \mu\left(1_{\bar{u}} \otimes \bar{R}_{u}\right)\right. \\
& =1_{\mu_{u} .}
\end{aligned}
$$

Substituting this into our calculation gives

$$
\begin{aligned}
\tilde{\mu}_{u, v}^{\bullet} & =\hat{R}_{v}^{*} \otimes 1_{\mu_{\bar{v} \otimes \bar{u}}} \circ 1_{\mu_{\bar{v}}} \otimes \tilde{\mu}_{v, \bar{v} \otimes \bar{u}}^{*} \circ 1_{\mu_{\bar{v}}} \otimes \mu\left(\bar{R}_{v} \otimes 1_{\bar{u}}\right) \\
& =\mu\left(R_{v}^{*}\right) \otimes 1_{\mu_{\bar{v} \otimes \bar{u}}} \circ \tilde{\mu}_{\bar{v}, v} \otimes 1_{\mu_{\bar{v} \otimes \bar{u}}} \circ 1_{\mu_{\bar{v}}} \otimes \tilde{\mu}_{v, \bar{v} \otimes \bar{u}^{*}}^{*} 1_{\mu_{\bar{v}}} \otimes \mu\left(\bar{R}_{v} \otimes 1_{\bar{u}}\right) \\
& =\mu\left(R_{v}^{*}\right) \otimes 1_{\mu_{\bar{v} \otimes \bar{u}}} \circ \tilde{\mu}_{\bar{v} \otimes v, \bar{v} \otimes u}^{*} \circ \tilde{\mu}_{\bar{v}, v \otimes \bar{v} \otimes \bar{u}} \circ 1_{\mu_{\bar{v}}} \otimes \mu\left(\bar{R}_{v} \otimes 1_{\bar{u}}\right) \\
& =\mu\left(R_{v}^{*} \otimes 1_{\bar{v} \otimes u} \circ \mu\left(1_{\bar{v}} \otimes \bar{R}_{v} \otimes 1_{u}\right) \circ \tilde{\mu}_{\bar{v}, \bar{u}}\right. \\
& =\tilde{\mu}_{\bar{v}, \bar{u} .}
\end{aligned}
$$

Dualizing with respect to $\otimes$ yields $\tilde{\mu}_{u, v}^{* \bullet *}=\tilde{\mu}_{\bar{v}, \bar{u}}$ and taking adjoints completes the proof.

Corollary 13.2. For $M \in\left(\mu_{u}, \mu_{u^{\prime}}\right), N \in\left(\mu_{v}, \mu_{v^{\prime}}\right)$,

$$
\left(\tilde{\mu}_{u^{\prime}, v^{\prime}} \circ M \otimes N \circ \tilde{\mu}_{u, v}^{*}\right)^{\bullet}=\tilde{\mu}_{\bar{v}^{\prime}, \bar{u}^{\prime}} \circ N^{\bullet} \otimes M^{\bullet} \circ \tilde{\mu}_{\bar{v}, \bar{u}}^{*},
$$

with respect to the image of a tensor product solution of the conjugate equations. 
Proof. By the previous proposition,

$$
\left(\tilde{\mu}_{u^{\prime}, v^{\prime}} \circ M \otimes N \circ \tilde{\mu}_{u, v}^{*}\right)^{\bullet}=\tilde{\mu}_{u^{\prime}, v^{\prime}}^{\bullet}(M \otimes N)^{\bullet} \circ \tilde{\mu}_{u, v}^{* \bullet}=\tilde{\mu}_{\overline{v^{\prime}, \bar{u}^{\prime}}} \circ N^{\bullet} \otimes M^{\bullet} \circ \tilde{\mu}_{\bar{v}, \bar{u} .}
$$

Proposition 13.3. If we take the conjugate solution $R_{\bar{u}}=\bar{R}_{u}$ as a solution of the conjugate equations for $\bar{u}$ and the tensor product solution $R_{\bar{u} \otimes u}=1_{\bar{u}} \otimes R_{\bar{u}} \otimes 1_{u} \circ R_{u}$ for $\bar{u} \otimes u$, then $R_{u}^{*}=R_{u}$ and $R_{u}^{* \bullet}=R_{u}^{*}$.

Proof. We have

$$
R_{u}^{\bullet}=R_{u}^{*} \otimes 1_{\bar{u} \otimes u} \circ \bar{R}_{\bar{u} \otimes u}=R_{u}^{*} \otimes 1_{\bar{u} \otimes u} \circ 1_{\bar{u}} \otimes \bar{R}_{u} \otimes 1_{u} \circ R_{u}=R_{u} .
$$

Dualizing again with respect to $\otimes$ gives $R_{u}^{* \bullet}=R_{u}^{*}$.

Acknowledgements. We are grateful to S. Doplicher for numerous discussions on an early stage of this line of research. He pursued the idea of embedding tensor $\mathrm{C}^{*}$-categories into categories of Hilbert bimodules.

Part of the results of this paper have been announced at a conference held in Leuven in the fall 2008. The first author would like to thank S. Vaes for the invitation and for discussions. She would also like to thank P. Hajac and R. Tomatsu for discussions.

Finally, we are grateful to the referee. His comments led to a simplified and improved presentation of the paper.

\section{References}

[1] S. Baaj and G. Skandalis, Unitaires multiplicatifs et dualité pour les produits croisés de $C^{*}$-algèbres. Ann. Sci. École Norm. Sup. (4) 26 (1993), 425-488. Zbl 0804.46078 MR 1235438

[2] J. Bichon, A. De Rijdt, and S. Vaes, Ergodic coactions with large multiplicity and monoidal equivalence of quantum groups. Comm. Math. Phys. 262 (2006), 703-728. Zbl 1122.46046 MR 2202309

[3] B. Blackadar, K-theory for operator algebras. Math. Sci. Res. Inst. Publ. 5, SpringerVerlag, New York 1986. Zbl 0597.46072 MR 859867

[4] F. P. Boca, Ergodic actions of compact matrix pseudogroups on $C^{*}$-algebras. Astérisque 232 (1995), 93-109. Zbl 0842.46039 MR 1372527

[5] G. Böhm, F. Nill, and K. Szlachányi, Weak Hopf algebras: I. Integral theory and $C^{*}$ structure. J. Algebra 221 (1999), 385-438. Zbl 0949.16037 MR 1726707

[6] A. Connes, Noncommutative geometry. Academic Press, San Diego, CA, 1994. Zbl 0818.46076 MR 1303779

[7] J. Cuntz, Regular actions of Hopf algebras on the $C^{*}$-algebra generated by a Hilbert space. In Operator algebras, mathematical physics, and low-dimensional topology (Istanbul, 1991), Res. Notes Math. 5, A K Peters, Wellesley, MA, 1993, 87-100. Zbl 0803.46062 MR 1259060 
[8] P. Deligne, Catégories tannakiennes. In The Grothendieck Festschrift, Vol. II, Progr. Math. 87, Birkhäuser, Boston 1990, 111-195. Zbl 0727.14010 MR 1106898

[9] S. Doplicher, C. Pinzari, and J. E. Roberts, An algebraic duality theory for multiplicative unitaries. Internat. J. Math. 12 (2001), 415-459. Zbl 1111.46312 MR 1841399

[10] S. Doplicher and J. E. Roberts, Duals of compact Lie groups realized in the Cuntz algebras and their actions on $C^{*}$-algebras. J. Funct. Anal. 74 (1987), 96-120. Zbl 0619.46053 MR 901232

[11] S. Doplicher and J. E. Roberts, A new duality theory for compact groups. Invent. Math. 98 (1989), 157-218. Zbl 0691.22002 MR 1010160

[12] P. Etingof and S. Gelaki, Isocategorical groups. Internat. Math. Res. Notices 2001 (2001), 59-76. Zbl 0988.20003 MR 1810480

[13] P. Etingof, D. Nikshych, and V. Ostrik, On fusion categories. Ann. of Math. (2) 162 (2005), 581-642. Zbl 1125.16025 MR 2183279

[14] T. Hayashi, A canonical Tannaka duality for finite semisimple tensor categories. Preprint 1999. arXiv:math/9904073

[15] R. Høegh-Krohn, M. B. Landstad, and E. Størmer, Compact ergodic groups of automorphisms. Ann. of Math. (2) 114 (1981), 75-86. Zbl 0472.46046 MR 625345

[16] M. Izumi, Subalgebras of infinite $C^{*}$-algebras with finite Watatani indices. I. Cuntz algebras. Comm. Math. Phys. 155 (1993), 157-182. Zbl 0803.46066 MR 1228532

[17] M. Izumi and H. Kosaki, On a subfactor analogue of the second cohomology. Rev. Math. Phys. 14 (2002), 733-757. Zbl 1030.46098 MR 1932664

[18] V. F. R. Jones, Index for subfactors. Invent. Math. 72 (1983), 1-25. Zbl 0508.46040 MR 696688

[19] A. Kirillov, Jr. and V. Ostrik, On a $q$-analogue of the McKay correspondence and the ADE classification of $\mathfrak{s l}_{2}$ conformal field theories. Adv. Math. 171 (2002), 183-227. Zbl 1024.17013 MR 1936496

[20] E. C. Lance, Hilbert $C^{*}$-modules. London Math. Soc. Lecture Note Ser. 210, Cambridge University Press, Cambridge 1995. Zbl 0822.46080 MR 1325694

[21] R. Longo, A duality for Hopf algebras and for subfactors. I. Comm. Math. Phys. 159 (1994), 133-150. Zbl 0802.46075 MR 1257245

[22] R. Longo and J. E. Roberts, A theory of dimension. K-Theory 11 (1997), 103-159. Zbl 0874.18005 MR 1444286

[23] G. W. Mackey, Induced representations of locally compact groups I. Ann. of Math. (2) 55 (1952), 101-139. Zbl 0046.11601 MR 0044536

[24] G. W. Mackey, Ergodic theory and virtual groups. Math. Ann. 166 (1966), 187-207. Zbl 0178.38802 MR 0201562

[25] S. MacLane, Categories for the working mathematician. Graduate Texts in Math. 5, Springer-Verlag, New York 1971. Zbl 0232.18001 MR 0354798

[26] D. Nikshych and L. Vainerman, A characterization of depth 2 subfactors of $\mathrm{II}_{1}$ factors. J. Funct. Anal. 171 (2000), 278-307. Zbl 1010.46063 MR 1745634

[27] D. Nikshych and L. Vainerman, A Galois correspondence for $\mathrm{II}_{1}$ factors and quantum groupoids. J. Funct. Anal. 178 (2000), 113-142. Zbl 0995.46041 MR 1800792 
[28] F. O'Cairbre, Full multiplicity ergodic actions of compact groups on von Neumann algebras. J. Operator Theory 29 (1993), 69-81. Zbl 0819.46052 MR 1277965

[29] A. Ocneanu, The classification of subgroups of quantum $\mathrm{SU}(N)$. In Quantum symmetries in theoretical physics and mathematics (Bariloche, 2000), Contemp. Math. 294, Amer. Math. Soc., Providence, RI, 2002, 133-159. Zbl 1193.81055 MR 1907188

[30] V. Ostrik, Module categories, weak Hopf algebras and modular invariants. Transform. Groups 8 (2003), 177-206. Zbl 1044.18004 MR 1976459

[31] A. Pal, Induced representation and Frobenius reciprocity for compact quantum groups. Proc. Indian Acad. Sci. Math. Sci. 105 (1995), 157-167. Zbl 0959.22004 MR 1350475

[32] C. Pinzari, The representation category of the Woronowicz quantum group $S_{\mu} U(d)$ as a braided tensor $C^{*}$-category. Internat. J. Math. 18 (2007), 113-136. Zbl 1120.46056 MR 2307417

[33] C. Pinzari, Embedding ergodic actions of compact quantum groups on $C^{*}$-algebras into quotient spaces. Internat. J. Math. 18 (2007), 137-164. Zbl 1120.46057 MR 2307418

[34] C. Pinzari and J. E. Roberts, A duality theorem for ergodic actions of compact quantum groups on $C^{*}$-algebras. Comm. Math. Phys. 277 (2008), 385-421. Zbl 1160.46045 MR 2358289

[35] C. Pinzari and J. E. Roberts, Ergodic actions of compact quantum groups from solutions of the conjugate equations. Preprint 2008. arXiv:0808.3326.

[36] C. Pinzari and J. E. Roberts, Ergodic actions of $S_{\mu} U(2)$ on $C^{*}$-algebras from $\mathrm{II}_{1}$ subfactors. J. Geom. Phys. 60 (2010), 403-416. Zbl 1195.46074 MR 2600003

[37] P. Podleś, Symmetries of quantum spaces. Subgroups and quotient spaces of quantum SU(2) and SO(3) groups. Comm. Math. Phys. 170 (1995), 1-20. Zbl 0853.46074 MR 1331688

[38] S. Popa, Classification of amenable subfactors of type II. Acta Math. 172 (1994), 163-255. Zbl 0853.46059 MR 1278111

[39] M. A. Rieffel, A global view of equivariant vector bundles and Dirac operators on some compact homogeneous spaces. In Group representations, ergodic theory, and mathematical physics: a tribute to George W. Mackey, Contemp. Math. 449, Amer. Math. Soc., Providence, RI, 2008, 399-415. Zbl 1163.53032 MR 2391813

[40] G. Segal, Equivariant K-theory. Inst. Hautes Études Sci. Publ. Math. 34 (1968), 129-151. Zbl 0199.26202 MR 0234452

[41] R. G. Swan, Vector bundles and projective modules. Trans. Amer. Math. Soc. 105 (1962), 264-277. Zbl 0109.41601 MR 0143225

[42] W. Szymański, Finite index subfactors and Hopf algebra crossed products. Proc. Amer. Math. Soc. 120 (1994), 519-528. Zbl 0802.46076 MR 1186139

[43] M. Takesaki, Duality for crossed products and the structure of von Neumann algebras of type III. Acta Math. 131 (1973), 249-310. Zbl 0268.46058 MR 0438149

[44] S. Vaes, A new approach to induction and imprimitivity results. J. Funct. Anal. 229 (2005), 317-374. Zbl 1087.22005 MR 2182592

[45] S. Wang, Ergodic actions of universal quantum groups on operator algebras. Comm. Math. Phys. 203 (1999), 481-498. Zbl 0967.46049 MR 1697607 
[46] A. Wassermann, Ergodic actions of compact groups on operator algebras. III. Classification for SU(2). Invent. Math. 93 (1988), 309-354. Zbl 0692.46058 MR 948104

[47] A. Wassermann, Ergodic actions of compact groups on operator algebras I. General theory. Ann. of Math. (2) 130 (1989), 273-319. Zbl 0734.46041 MR 1014926

[48] H. Wenzl, Hecke algebras of type $A_{n}$ and subfactors. Invent. Math. 92 (1988), 349-383. Zbl 0663.46055 MR 936086

[49] S. L. Woronowicz, Compact matrix pseudogroups. Comm. Math. Phys. 111 (1987), 613-665. Zbl 0627.58034 MR 901157

[50] S. L. Woronowicz, Tannaka-Krein duality for compact matrix pseudogroups. Twisted SU(N) groups. Invent. Math. 93 (1988), 35-76. Zbl 0664.58044 MR 943923

[51] S. L. Woronowicz, Compact quantum groups. In Symétries quantiques/Quantum symmetries (Les Houches, 1995), North-Holland, Amsterdam 1998, 845-884. Zbl 0997.46045 MR 1616348

Received May 14, 2010; revised October 7, 2010

C. Pinzari, Dipartimento di Matematica, Università di Roma “La Sapienza”, 00185 Roma, Italy

E-mail: pinzari@mat.uniroma1.it

J. E. Roberts, Dipartimento di Matematica, Università di Roma “Tor Vergata”, 00133 Roma, Italy

E-mail: roberts@mat.uniroma2.it 